TRANSACTIONS OF THE

AMERICAN MATHEMATICAL SOCIETY

Volume 360, Number 7, July 2008, Pages 3429-3472

S 0002-9947(08)04373-0

Article electronically published on February 13, 2008

\title{
BRAIDED SYMMETRIC AND EXTERIOR ALGEBRAS
}

\author{
ARKADY BERENSTEIN AND SEBASTIAN ZWICKNAGL
}

\begin{abstract}
The goal of the paper is to introduce and study symmetric and exterior algebras in certain braided monoidal categories such as the category $\mathcal{O}$ for quantum groups. We relate our braided symmetric algebras and braided exterior algebras with their classical counterparts.
\end{abstract}

\section{INTRODUCTION}

The goal of the paper is to introduce and study symmetric and exterior algebras in certain braided monoidal categories of modules over quantum groups and compare these braided symmetric algebras and braided exterior algebras with their classical counterparts.

The original motivation of this work comes from the following classical problem.

Problem 0.1. Let $V$ be a simple finite-dimensional module over a complex semisimple Lie algebra $\mathfrak{g}$; find the decomposition of symmetric and exterior powers of $V$ into simple $\mathfrak{g}$-modules.

This problem is open and, to some extent, not settled even for $\mathfrak{g}=s l_{2}(\mathbb{C})$. A modern approach to the problem would consist of constructing a basis (which can be referred to as a crystal basis or a canonical basis) in the symmetric algebra $S(V)$ (or in the exterior algebra $\Lambda(V)$ ) which is somehow compatible with the irreducible submodules. Typically, the crystal (or canonical) bases require some kind of $q$ deformation of the involved modules and algebras. Following Lusztig's original idea of [17], in order to implement this program, one should first $q$-deform $S(V)$ (or $\Lambda(V)$ ) and then try to construct the canonical (or crystal) basis for $S_{q}(V)$ (or $\left.\Lambda_{q}(V)\right)$ using a "bar"-involution and some standard PBW-like basis.

Therefore, the following problem naturally arises.

Problem 0.2. Let $U_{q}(\mathfrak{g})$ be the quantized enveloping algebra of $\mathfrak{g}$. For each finite dimensional $U_{q}(\mathfrak{g})$-module $V$ construct (in the the category of $U_{q}(\mathfrak{g})$-module algebras) an analogue $S_{q}(V)$ of the symmetric algebra of $V$ and an analogue $\Lambda_{q}(V)$ of the exterior algebra of $V$.

Apparently, the first work in which Problem 0.2 was mentioned is the paper 19. Its main result asserts that if $V$ is the 4-dimensional simple $U_{q}\left(s l_{2}(\mathbb{C})\right)$-module, then the algebra $S_{q}(V)$ cannot be a flat deformation of $S(V)$. Unfortunately, the results of [19 neither suggested a solution to the problem nor generated a followup research. For the case when $V$ is the quantum adjoint module over $U_{q}\left(s l_{n}(\mathbb{C})\right)$,

Received by the editors November 9, 2005.

2000 Mathematics Subject Classification. Primary 17B37; Secondary 17B63.

This research was supported in part by NSF grants \#DMS-0102382 and \#DMS-0501103.

(C)2008 American Mathematical Society

Reverts to public domain 28 years from publication 
a version of $S_{q}(V)$ was constructed by J. Donin in [11. Another, yet unrelated approach, based on Woronowicz's quantum differential calculus, associates to $V$ an algebra $\mathcal{B}(V)$ (which is sometimes referred to as "quantum symmetric algebra" or Nichols-Woronowicz algebra, see e.g., [2, 12, 18, 20]). However, $\mathcal{B}(V)$ is typically much larger than the ordinary symmetric algebra $S(V)$.

We propose the following construction of $S_{q}(V)$. First, recall that the category $\mathcal{O}_{f}$ of finite-dimensional $U_{q}(\mathfrak{g})$-modules is braided monoidal, where the braiding $\mathcal{R}_{U, V}: U \otimes V \rightarrow V \otimes U$ is the permutation of factors composed with the universal $R$-matrix.

For each object $V$ of $\mathcal{O}_{f}$ define the quantum exterior square $\Lambda_{q}^{2} V \subset V \otimes V$ to be the linear span of all negative eigenvectors of $\mathcal{R}_{V, V}: V \otimes V \rightarrow V \otimes V$, that is, the eigenvectors that correspond to negative (i.e., having the form $-q^{r}, r \in \mathbb{Z}$ ) eigenvalues of $\mathcal{R}$. Clearly, $\Lambda_{q}^{2} V$ is a well-defined flat deformation of $\Lambda^{2} V$. Note that this definition makes sense because the square $\left(\mathcal{R}_{V, V}\right)^{2}$ is a diagonalizable linear map $V \otimes V \rightarrow V \otimes V$ whose eigenvalues are powers of $q$.

We define the braided symmetric algebra $S_{q}(V)$ of $V$ as the quotient of the tensor algebra $T(V)$ by the ideal generated by $\Lambda_{q}^{2} V$. By the very definition the correspondence $V \mapsto S_{q}(V)$ is a functor from the category $\mathcal{O}_{f}$ to the category of graded $U_{q}(\mathfrak{g})$-module algebras.

We prove (see Theorem 2.21) that the braided symmetric and exterior powers are "less or equal" than their classical counterparts, which motivates the following definition. We say that a module $V$ is flat if $S_{q}(V)$ is a flat deformation of the ordinary symmetric algebra $S(V)$.

Problem 0.3. Classify all flat modules in $\mathcal{O}_{f}$.

In section 2.3 (Proposition 2.31) we provide a sufficient criterion for flatness, and numerous examples convince us that this criterion is also necessary. Among the examples of flat modules is the standard $n$-dimensional $U_{q}\left(s l_{n}(\mathbb{C})\right.$ )-module $V$, and its braided symmetric algebra is isomorphic to the algebra of $q$-polynomials in $n$ variables. In this paper we completely classify flat simple $U_{q}\left(s l_{2}(\mathbb{C})\right)$-modules and prove that a simple $n$-dimensional $U_{q}\left(s l_{2}(\mathbb{C})\right)$-module is flat if and only if $n=1,2,3$. The fact that the "adjoint" (i.e., 3 -dimensional simple) module $V$ is flat agrees with the results of 1 .

A general expectation is that for each $\mathfrak{g}$ there is a finite set of dominant weights $\lambda$ such that the irreducible $U_{q}(\mathfrak{g})$-module $V_{\lambda}$ is flat. Informally speaking, we expect that each "small enough" irreducible $U_{q}(\mathfrak{g})$-module is flat. A complete classification of flat modules has recently been obtained in 22 .

Let us reiterate that our immediate motivation for introducing $S_{q}(V)$ and $\Lambda_{q}(V)$ is to prepare the ground for a general notion of the canonical basis. It turns out that for each flat $U_{q}$-module $V$ from the list compiled in [22, the algebra $S_{q}(V)$ is isomorphic to the associated graded algebra of the quantized enveloping algebra $U_{q}(\mathfrak{u})$ (where $\mathfrak{u}$ is a certain nilpotent Lie algebra) and, therefore, $S_{q}(V)$ carries a canonical basis which can be defined along the lines of [17. In its turn, this defines a crystal basis for the ordinary symmetric algebra $S(\bar{V})$, where $\bar{V}$ is the corresponding $\mathfrak{g}$-module and, therefore, solves Problem 0.1. A surprising application of this crystal basis (and, in fact, another motivation of the project) is a construction of a geometric crystal on $\bar{V}$ (see [3, 4, 5, 6]).

A more ambitious goal is to study braided symmetric algebras of non-flat modules. Already for $\mathfrak{g}=s l_{2}(\mathbb{C})$ we obtained an unexpected result for each simple 
module $V$ : the braided symmetric cube $S_{q}^{3} V$ is multiplicity-free as a $U_{q}\left(s l_{2}(\mathbb{C})\right.$ )module (Theorem 2.35). Numerous examples convince us that this phenomenon should take place for any braided symmetric power $S_{q}^{n} V_{\ell}$ (Conjecture 2.37). Our general expectation is that the braided symmetric powers of finite dimensional $U_{q}(\mathfrak{g})$-modules have much nicer decompositions into the irreducibles than their classical counterparts and, therefore, the "braided" version of the above Problem 0.1 is much easier to solve.

In a similar manner we define the braided exterior algebra $\Lambda_{q}(V)$ to be the quotient of the tensor algebra $T(V)$ by the ideal generated by the braided symmetric square $S_{q}^{2} V$. Similarly to the ordinary symmetric and exterior algebras, one has a quadratic duality between the quadratic algebras $S_{q}(V)$ and $\Lambda_{q}\left(V^{*}\right)$. Again, similarly to the classical case, for each finite-dimensional module $V$ its braided exterior algebra is also finite dimensional.

The structure of braided exterior algebras for $U_{q}\left(s l_{2}(\mathbb{C})\right)$-modules is more transparent: we prove that $\Lambda_{q}^{4} V=0$ for any simple $U_{q}\left(s l_{2}(\mathbb{C})\right)$-module $V$ and, moreover, if $n=\operatorname{dim} V$ is even, then $\Lambda_{q}^{3} V=0$.

Needless to say, this paper is only a starting point in exploring the new braided algebras, and we expect many more interesting results in their study.

\section{Definitions And notation}

We start with the definition of the quantized enveloping algebra associated with a complex reductive Lie algebra $\mathfrak{g}$ (our standard reference here will be [10]). Let $\mathfrak{h} \subset \mathfrak{g}$ be the Cartan subalgebra, and let $A=\left(a_{i j}\right)$ be the Cartan matrix for $\mathfrak{g}$.

Definition 1.1. A realization of $A$ is a triple $(P, \Pi,(\cdot \mid \cdot)$ ), where $P$ is a lattice (of full dimension) in $\mathfrak{h}^{*}, \Pi=\left\{\alpha_{1}, \ldots, \alpha_{r}\right\} \subset P$, is a subset, and $(\cdot \mid \cdot)$ is a $\mathbb{Z}$-valued inner product on $P$ satisfying the following conditions:

- $\Pi$ is linearly independent.

- $\frac{2\left(\lambda \mid \alpha_{i}\right)}{\left(\alpha_{i} \mid \alpha_{i}\right)} \in \mathbb{Z}$ and $\frac{\left(\alpha_{i} \mid \alpha_{i}\right)}{2} \in \mathbb{Z}_{\geq 0}$ for all $\lambda \in P, i \in[1, r]$.

- $\frac{2\left(\alpha_{i} \mid \alpha_{j}\right)}{\left(\alpha_{i} \mid \alpha_{i}\right)}=a_{i j}$ for all $i, j$.

The quantized enveloping algebra $U$ is a $\mathbb{C}(q)$-algebra generated by the elements $E_{i}$ and $F_{i}$ for $i \in[1, r]$, and $K_{\lambda}$ for $\lambda \in P$, subject to the following relations: $K_{\lambda} K_{\mu}=K_{\lambda+\mu}, K_{0}=1$ for $\lambda, \mu \in P ; K_{\lambda} E_{i}=q^{\left(\alpha_{i} \mid \lambda\right)} E_{i} K_{\lambda}, K_{\lambda} F_{i}=q^{-\left(\alpha_{i} \mid \lambda\right)} F_{i} K_{\lambda}$ for $i \in[1, r]$ and $\lambda \in P$;

$$
E_{i}, F_{j}-F_{j} E_{i}=\delta_{i j} \frac{K_{\alpha_{i}}-K_{-\alpha_{i}}}{q^{d_{i}}-q^{-d_{i}}}
$$

for $i, j \in[1, r]$, where $d_{i}=\frac{\left(\alpha_{i} \mid \alpha_{i}\right)}{2}$; and the quantum Serre relations

$$
\sum_{p=0}^{1-a_{i j}}(-1)^{p} E_{i}^{\left(1-a_{i j}-p\right)} E_{j} E_{i}^{(p)}=0, \sum_{p=0}^{1-a_{i j}}(-1)^{p} F_{i}^{\left(1-a_{i j}-p\right)} F_{j} F_{i}^{(p)}=0
$$

for $i \neq j$, where the notation $X_{i}^{(p)}$ stands for the divided power

$$
X_{i}^{(p)}=\frac{X^{p}}{(1)_{i} \cdots(p)_{i}}, \quad(k)_{i}=\frac{q^{k d_{i}}-q^{-k d_{i}}}{q^{d_{i}}-q^{-d_{i}}} .
$$

The algebra $U$ is a $q$-deformation of the universal enveloping algebra of the reductive Lie algebra $\mathfrak{g}$ associated to $A$, so it is commonly denoted by $U=U_{q}(\mathfrak{g})$. 
It has a natural structure of a bialgebra with the co-multiplication $\Delta: U \rightarrow U \otimes U$ and the counit homomorphism $\varepsilon: U \rightarrow \mathbb{Q}(q)$ given by

$$
\begin{gathered}
\Delta\left(E_{i}\right)=E_{i} \otimes 1+K_{\alpha_{i}} \otimes E_{i}, \Delta\left(F_{i}\right)=F_{i} \otimes K_{-\alpha_{i}}+1 \otimes F_{i}, \Delta\left(K_{\lambda}\right)=K_{\lambda} \otimes K_{\lambda}, \\
\varepsilon\left(E_{i}\right)=\varepsilon\left(F_{i}\right)=0, \quad \varepsilon\left(K_{\lambda}\right)=1 .
\end{gathered}
$$

In fact, $U$ is a Hopf algebra with the antipode anti-homomorphism $S: U \rightarrow U$ given by $S\left(E_{i}\right)=-K_{-\alpha_{i}} E_{i}, S\left(F_{i}\right)=-F_{i} K_{\alpha_{i}}, S\left(K_{\lambda}\right)=K_{-\lambda}$.

Let $U^{-}$(resp. $U^{0} ; U^{+}$) be the $\mathbb{Q}(q)$-subalgebra of $U$ generated by $F_{1}, \ldots, F_{r}$ (resp. by $K_{\lambda}(\lambda \in P)$; by $\left.E_{1}, \ldots, E_{r}\right)$. It is well-known that $U=U^{-} \cdot U^{0} \cdot U^{+}$(more precisely, the multiplication map induces an isomorphism $U^{-} \otimes U^{0} \otimes U^{+} \rightarrow U$ ).

We will consider the full sub-category $\mathcal{O}_{f}$ of the category $U_{q}(\mathfrak{g})-\operatorname{Mod}$. The objects of $\mathcal{O}_{f}$ are finite-dimensional $U_{q}(\mathfrak{g})$-modules $V$ having a weight decomposition

$$
V=\bigoplus_{\mu \in P} V(\mu)
$$

where each $K_{\lambda}$ acts on each weight space $V(\mu)$ by the multiplication with $q^{(\lambda \mid \mu)}$ (see e.g., [10, I.6.12]). The category $\mathcal{O}_{f}$ is semisimple, and the irreducible objects $V_{\lambda}$ are generated by highest weight spaces $V_{\lambda}(\lambda)=\mathbb{C}(q) \cdot v_{\lambda}$, where $\lambda$ is a dominant weight, i.e., $\lambda$ belongs to $P^{+}=\left\{\lambda \in P:\left(\lambda \mid \alpha_{i}\right) \geq 0 \forall i \in[1, r]\right\}$, the monoid of dominant weights.

Let $R \in U_{q}(\mathfrak{g}) \hat{\otimes} U_{q}(\mathfrak{g})$ be the universal $R$-matrix. By definition,

$$
R=R_{0} R_{1}=R_{1} R_{0}
$$

where $R_{0}$ is "the diagonal part" of $R$, and $R_{1}$ is unipotent, i.e., $R_{1}$ is a formal power series

$$
R_{1}=1 \otimes 1+(q-1) x_{1}+(q-1)^{2} x_{2}+\cdots,
$$

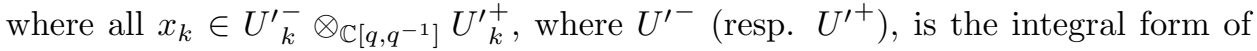
$U^{+}$, i.e., $U^{\prime-}$ is a $\mathbb{C}\left[q, q^{-1}\right]$-subalgebra of $U_{q}(\mathfrak{g})$ generated by all $F_{i}$ (resp. by all $E_{i}$ ) and $U_{k}^{\prime-}\left(\right.$ resp. $\left.U_{k}^{\prime+}\right)$, is the $k$-th graded component under the grading $\operatorname{deg}\left(F_{i}\right)=1$ $\left(\right.$ resp. $\left.\operatorname{deg}\left(E_{i}\right)=1\right)$.

By definition, for any $U, V$ in $\mathcal{O}_{f}$ and any highest weights elements $v_{\lambda} \in U(\lambda)$, $v_{\mu} \in V(\mu)$ we have $R_{0}\left(v_{\lambda} \otimes v_{\mu}\right)=q^{(\lambda \mid \mu)} v_{\lambda} \otimes v_{\mu}$.

Let $R^{o p}$ be the opposite element of $R$, i.e., $R^{o p}=\tau(R)$, where $\tau: U_{q}(\mathfrak{g}) \widehat{\otimes} U_{q}(\mathfrak{g})$ is the permutation of factors. Clearly, $R^{o p}=R_{0} R_{1}^{o p}=R_{1}^{o p} R_{0}$.

Following [13, Section 3], define $D \in U_{q}(\mathfrak{g}) \widehat{\otimes} U_{q}(\mathfrak{g})$ by

$$
D:=R_{0} \sqrt{R_{1}^{o p} R_{1}}=\sqrt{R_{1}^{o p} R_{1}} R_{0} .
$$

Clearly, $D$ is well-defined because $R_{1}^{o p} R_{1}$ is also unipotent as well as its square root. By definition, $D^{2}=R^{o p} R, D^{o p} R=R D$.

Furthermore, define

$$
\widehat{R}:=R D^{-1}=\left(D^{o p}\right)^{-1} R=R_{1}\left(\sqrt{R_{1}^{o p} R_{1}}\right)^{-1} .
$$

It is easy to see that

$$
\widehat{R}^{o p}=\widehat{R}^{-1} .
$$

According to [13, Proposition 3.3], the pair $\left(U_{q}(\mathfrak{g}), \widehat{R}\right)$ is a coboundary Hopf algebra. 
The braiding in the category $\mathcal{O}_{f}$ is defined by $\mathcal{R}_{U, V}: U \otimes V \rightarrow V \otimes U$, where

$$
\mathcal{R}_{U, V}(u \otimes v)=\tau R(u \otimes v)
$$

for any $u \in U, v \in V$, where $\tau: U \otimes V \rightarrow V \otimes U$ is the ordinary permutation of factors.

Denote by $C \in Z\left(\widehat{U_{q}(\mathfrak{g})}\right)$ the quantum Casimir element which acts on any irreducible $U_{q}(\mathfrak{g})$-module $V_{\lambda}$ in $\mathcal{O}_{f}$ by the scalar multiple $q^{(\lambda \mid \lambda+2 \rho)}$, where $2 \rho$ is the sum of positive roots.

The following fact is well-known.

Lemma 1.2. One has $\mathcal{R}^{2}=\Delta\left(C^{-1}\right) \circ(C \otimes C)$. In particular, for each $\lambda, \mu, \nu \in P_{+}$ the restriction of $\mathcal{R}^{2}$ to the $\nu$-th isotypic component $I_{\lambda, \mu}^{\nu}$ of the tensor product $V_{\lambda} \otimes V_{\mu}$ is scalar multiplication by $q^{((\lambda \mid \lambda)+(\mu \mid \mu)-(\nu \mid \nu))+(2 \rho \mid \lambda+\mu-\nu)}$.

This allows one to define the diagonalizable $\mathbb{C}(q)$-linear map $D_{U, V}: U \otimes V \rightarrow$ $U \otimes V$ by $D_{U, V}(u \otimes v)=D(u \otimes v)$ for any objects $U$ and $V$ of $\mathcal{O}_{f}$. It is easy to see that the operator $D_{V_{\lambda}, V_{\mu}}: V_{\lambda} \otimes V_{\mu} \rightarrow V_{\lambda} \otimes V_{\mu}$ acts on the $\nu$-th isotypic component $I_{\lambda, \mu}^{\nu}$ in $V_{\lambda} \otimes V_{\mu}$ by the scalar multiplication with $q^{\frac{1}{2}((\lambda \mid \lambda)+(\mu \mid \mu)-(\nu \mid \nu))+(\rho \mid \lambda+\mu-\nu)}$.

For any $U$ and $V$ in $\mathcal{O}_{f}$ define the normalized braiding $\sigma_{U, V}$ by

$$
\sigma_{U, V}(u \otimes v)=\tau \widehat{R}(u \otimes v) .
$$

Therefore, we have by (1.9):

$$
\sigma_{U, V}=D_{V, U}^{-1} \mathcal{R}_{U, V}=\mathcal{R}_{U, V} D_{U, V}^{-1}
$$

We will will sometimes write $\sigma_{U, V}$ in a more explicit way:

$$
\sigma_{U, V}=\sqrt{\mathcal{R}_{V, U}^{-1} \mathcal{R}_{U, V}^{-1}} \mathcal{R}_{U, V}=\mathcal{R}_{U, V} \sqrt{\mathcal{R}_{U, V}^{-1} \mathcal{R}_{V, U}^{-1}}
$$

The following fact is an obvious corollary of (1.10).

Lemma 1.3. $\sigma_{V, U} \circ \sigma_{U, V}=i d_{U \otimes V}$ for any $U, V$ in $\mathcal{O}_{f}$. That is, $\sigma$ is a symmetric commutativity constraint.

We also have the following coboundary relation (even though we will not use it).

Lemma 1.4 ([13, section 3]). Let $A, B, C$ be objects of $\mathcal{O}_{f}$. Then, the following diagram commutes:

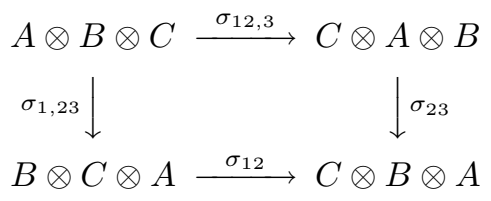

where we abbreviated

$$
\begin{aligned}
\sigma_{12,3} & :=\sigma_{A \otimes B, C}:(A \otimes B) \otimes C \rightarrow C \otimes(A \otimes B), \\
\sigma_{1,23} & :=\sigma_{A, B \otimes C}: A \otimes(B \otimes C) \rightarrow(B \otimes C) \otimes A .
\end{aligned}
$$

Remark 1.5. If one replaces the braiding $\mathcal{R}$ of $\mathcal{O}_{f}$ by its inverse $\mathcal{R}^{-1}$, the symmetric commutativity constraint $\sigma$ will not change. 


\section{MAIN RESULTS}

2.1. Braided symmetric and exterior powers. In this section we will use the notation and conventions of Section 1 .

For any morphism $f: V \otimes V \rightarrow V \otimes V$ in $\mathcal{O}_{f}$ and $n>1$ we denote by $f^{i, i+1}$, $i=1,2, \ldots, n-1$, the morphism $V^{\otimes n} \rightarrow V^{\otimes n}$ which acts as $f$ on the $i$-th and the $i+1$-st factors. Note that $\sigma_{V, V}^{i, i+1}$ is always an involution on $V^{\otimes n}$.

Definition 2.1. For an object $V$ in $\mathcal{O}_{f}$ and $n \geq 0$ define the braided symmetric power $S_{\sigma}^{n} V \subset V^{\otimes n}$ and the braided exterior power $\Lambda_{\sigma}^{n} V \subset V^{\otimes n}$ by:

$$
\begin{aligned}
& S_{\sigma}^{n} V=\bigcap_{1 \leq i \leq n-1}\left(\operatorname{Ker} \sigma_{i, i+1}-i d\right)=\bigcap_{1 \leq i \leq n-1}\left(\operatorname{Im} \sigma_{i, i+1}+i d\right), \\
& \Lambda_{\sigma}^{n} V=\bigcap_{1 \leq i \leq n-1}\left(\operatorname{Ker} \sigma_{i, i+1}+i d\right)=\bigcap_{1 \leq i \leq n-1}\left(\operatorname{Im} \sigma_{i, i+1}-i d\right),
\end{aligned}
$$

where we abbreviated $\sigma_{i, i+1}=\sigma_{V, V}^{i, i+1}$.

Remark 2.2. Clearly, $-\mathcal{R}$ is also a braiding on $\mathcal{O}_{f}$ and $-\sigma$ is the corresponding normalized braiding. Therefore, $\Lambda_{\sigma}^{n} V=S_{-\sigma}^{n} V$ and $S_{\sigma}^{n} V=\Lambda_{-\sigma}^{n} V$. That is, informally speaking, the symmetric and exterior powers are mutually "interchangeable".

Remark 2.3. Another way to introduce the symmetric and exterior squares involves the well-known fact that the braiding $\mathcal{R}_{V, V}$ is a semisimple operator $V \otimes V \rightarrow V \otimes V$, and all the eigenvalues of $\mathcal{R}_{V, V}$ are of the form $\pm q^{r}$, where $r \in \mathbb{Z}$. Then positive eigenvectors of $\mathcal{R}_{V, V}$ span $S_{\sigma}^{2} V$ and negative eigenvectors of $\mathcal{R}_{V, V}$ span $\Lambda_{\sigma}^{2} V$.

Clearly, $S_{\sigma}^{0} V=\mathbb{C}(q), S_{\sigma}^{1} V=V, \Lambda_{\sigma}^{0} V=\mathbb{C}(q), \Lambda_{\sigma}^{1} V=V$, and

$$
S_{\sigma}^{2} V=\left\{v \in V \otimes V \mid \sigma_{V, V}(v)=v\right\}, \Lambda_{\sigma}^{2} V=\left\{v \in V \otimes V \mid \sigma_{V, V}(v)=-v\right\} .
$$

We can provide a uniform characterization of braided symmetric and exterior powers as follows.

Definition 2.4. Let $V$ be an object of $\mathcal{O}_{f}$ and $I$ be a sub-object of $V \otimes V$ in $\mathcal{O}_{f}$. For each $n \geq 2$ define the braided power $P(I)^{n} \subset V^{\otimes n}$ of $I$ by:

$$
P^{n}(I)=\bigcap_{1 \leq i \leq n-1} V^{\otimes i-1} \otimes I \otimes V^{\otimes n-1-i}
$$

(and set $\left.P^{0}(I)=\mathbb{C}(q), P^{1}(I)=V\right)$.

The following fact is obvious.

Lemma 2.5. For each $n \geq 0$ we have $S_{\sigma}^{n} V=P^{n}\left(S_{\sigma}^{2} V\right)$ and $\Lambda_{\sigma}^{n} V=P^{n}\left(\Lambda_{\sigma}^{2}\right)$.

The following fact is obvious.

Proposition 2.6. For each $n \geq 0$ the association $V \mapsto S_{\sigma}^{n} V$ is a functor from $\mathcal{O}_{f}$ to $\mathcal{O}_{f}$ and the association $V \mapsto \Lambda_{\sigma}^{n} V$ is a functor from $\mathcal{O}_{f}$ to $\mathcal{O}_{f}$. In particular, an embedding $U \hookrightarrow V$ in the category $\mathcal{O}_{f}$ induces injective morphisms

$$
S_{\sigma}^{n} U \hookrightarrow S_{\sigma}^{n} V, \Lambda_{\sigma}^{n} U \hookrightarrow \Lambda_{\sigma}^{n} V .
$$

Definition 2.7. For any $V \in O b(\mathcal{O})$ define the braided symmetric algebra $S_{\sigma}(V)$ and the braided exterior algebra $\Lambda_{\sigma}(V)$ by:

$$
S_{\sigma}(V)=T(V) /\left\langle\Lambda_{\sigma}^{2} V\right\rangle, \Lambda_{\sigma}(V)=T(V) /\left\langle S_{\sigma}^{2} V\right\rangle
$$


where $T(V)$ is the tensor algebra of $V$ and $\langle I\rangle$ stands for the two-sided ideal in $T(V)$ generated by a subset $I \subset T(V)$.

Note that the algebras $S_{\sigma}(V)$ and $\Lambda_{\sigma}(V)$ carry a natural $\mathbb{Z}_{\geq 0}$-grading:

$$
S_{\sigma}(V)=\bigoplus_{n \geq 0} S_{\sigma}(V)_{n}, \Lambda_{\sigma}(V)=\bigoplus_{n \geq 0} \Lambda_{\sigma}(V)_{n}
$$

since the respective ideals in $T(V)$ are homogeneous.

Denote by $\mathcal{O}_{g r, f}$ the sub-category of $U_{q}(\mathfrak{g})-M o d$ whose objects are $\mathbb{Z}_{\geq 0}$-graded:

$$
V=\bigoplus_{n \in \mathbb{Z}_{\geq 0}} V_{n}
$$

where each $V_{n}$ is an object of $\mathcal{O}_{f}$; and morphisms are those homomorphisms of $U_{q}(\mathfrak{g})$-modules which preserve the $\mathbb{Z}_{\geq 0}$-grading.

Clearly, $\mathcal{O}_{g r, f}$ is a tensor category under the natural extension of the tensor structure of $\mathcal{O}_{f}$. Therefore, we can speak of algebras and coalgebras in $\mathcal{O}_{g r, f}$.

By the very definition, $S_{\sigma}(V)$ and $\Lambda_{\sigma}(V)$ are algebras in $\mathcal{O}_{g r, f}$.

Proposition 2.8. The assignments $V \mapsto S_{\sigma}(V)$ and $V \mapsto \Lambda_{\sigma}(V)$ define functors from $\mathcal{O}_{f}$ to the category of algebras in $\mathcal{O}_{g r, f}$.

Let $V$ be an object of $\mathcal{O}_{f}$ and $I^{2}$ be a sub-object of $V \otimes V$ in $\mathcal{O}_{f}$ as in Definition 2.4. Using the identity $P^{m}(I) \otimes V^{\otimes n} \cap V^{\otimes m} \otimes P^{n}(I)=P^{m}(I) \otimes P^{n}(I)$ and natural inclusions $P^{m+n}(I) \subset P^{m}(I) \otimes V^{\otimes n}, P^{m+n}(I) \subset V^{\otimes m} \otimes P^{n}(I)$, we obtain natural embeddings $\Delta_{m, n}^{I}: P^{m+n}(I) \hookrightarrow P^{m}(I) \otimes P^{n}(I)$ in the category $\mathcal{O}_{f}$.

Let $P(I):=\bigoplus_{n \geq 0} P^{n}(I)$; define the morphism $\Delta_{I}: P(I) \rightarrow P(I) \otimes P(I)$ by $\Delta^{I}=\bigoplus_{n, m \geq 0} \Delta_{m, n}^{I}$, and let $\varepsilon_{I}$ be the natural projection $P(I) \rightarrow P^{0}(I)=\mathbb{C}(q)$.

Lemma 2.9. The triple $\left(P(I), \Delta^{I}, \varepsilon_{I}\right)$ is a coalgebra in $\mathcal{O}_{g r, f}$.

Proof. Clearly, $\Delta_{I}$ is co-associative. The compatibility between the co-multiplication and the co-unit also follows.

Corollary 2.10. For each object $V$ of $\mathcal{O}_{f}$ the braided symmetric space $S_{\sigma} V=$ $P\left(S_{\sigma}^{2} V\right)$ and the braided exterior space $\Lambda_{\sigma} V=P\left(\Lambda_{\sigma}^{2} V\right)$ are naturally co-algebras in $\mathcal{O}_{f}$. The assignments $V \mapsto S_{\sigma} V$ and $V \mapsto \Lambda_{\sigma} V$ define functors from $\mathcal{O}_{f}$ to the category of coalgebras in $\mathcal{O}_{g r, f}$.

Note that both $\mathcal{O}_{f}$ and $\mathcal{O}_{g r, f}$ are categories with the duality *.

Proposition 2.11. For any $V$ in $\mathcal{O}_{f}$ we have the following isomorphisms in $\mathcal{O}_{g r, f}$ :

(a) Algebra isomorphisms: $\left(S_{\sigma} V\right)^{*} \cong S_{\sigma}\left(V^{*}\right),\left(\Lambda_{\sigma} V\right)^{*} \cong \Lambda_{\sigma}\left(V^{*}\right)$.

(b) Coalgebra isomorphisms: $\left(S_{\sigma}(V)\right)^{*} \cong S_{\sigma} V^{*},\left(\Lambda_{\sigma}(V)\right)^{*} \cong \Lambda_{\sigma} V^{*}$.

(c) Algebra isomorphisms: $\Lambda_{\sigma}(V)^{!} \cong S_{\sigma}\left(V^{*}\right), S_{\sigma}(V)^{!} \cong \Lambda_{\sigma}\left(V^{*}\right)$, where $A^{!}$stands for the quadratic dual of a quadratic algebra $A$ in $\mathcal{O}_{g r, f}$.

Proof. Follows from the fact that $T(V)^{*} \cong T\left(V^{*}\right)$ in $\mathcal{O}_{g r, f},\left(\Lambda_{\sigma}^{2} V\right)^{\perp}=S_{\sigma}^{2} V^{*}$, $\left(S_{\sigma}^{2} V\right)^{\perp}=\Lambda_{\sigma}^{2} V^{*}$ in $\mathcal{O}_{f}$ and the general fact that for any $I, J \subset V \otimes V$ such that $I+J=V \otimes V$ and $I \cap J=0$ one has:

$$
P(I)^{\perp}=\left\langle J^{\perp}\right\rangle, P(J)^{\perp}=\left\langle I^{\perp}\right\rangle
$$

in $\mathcal{O}_{g r, f}$, where $\langle X\rangle$ is the ideal in $T(V)^{*}$ generated by $X \subset(V \otimes V)^{*}=V^{*} \otimes V^{*}$. 
In particular, Proposition 2.11(c) asserts that the relationship between the braided symmetric (resp. exterior) powers and the corresponding components of braided symmetric (resp. exterior) algebras is given by the following canonical isomorphisms (for all $n \geq 2$ ):

$$
\left(S_{\sigma}^{n} V^{*}\right)^{*} \cong S_{\sigma}(V)_{n},\left(\Lambda_{\sigma}^{n} V^{*}\right)^{*} \cong \Lambda_{\sigma}(V)_{n} .
$$

To discuss a possibility of a direct isomorphism, we propose the following problem.

Problem 2.12. Given $V$ in $\mathcal{O}_{f}$, find all the pairs of sub-objects $I, J \subset V \otimes V$ such that $I+J=V \otimes V, I \cap J=0$, and the composition of the natural inclusion $P(I) \hookrightarrow$ $T(V)$ with the structure homomorphism $T(V) \rightarrow T(V) /\langle J\rangle$ is an isomorphism $P(I) \cong T(V) /\langle J\rangle$ in $\mathcal{O}_{g r, f}$.

We would expect that the pairs $(I, J)=\left(S_{\sigma}^{2} V, \Lambda_{\sigma}^{2} V\right)$ and $(I, J)=\left(\Lambda_{\sigma}^{2} V, S_{\sigma}^{2} V\right)$ are solutions to Problem 2.12 for any $V \in \mathcal{O}_{f}$. This expectation is certainly true whenever when $\mathfrak{g}$ is abelian, i.e., when $\sigma=\tau$, and $S_{\sigma}(V)=S(V), \Lambda_{\sigma}(V)=\Lambda(V)$.

Proposition 2.13. For any $V$ in $\mathcal{O}_{f}$ each embedding $V_{\lambda} \hookrightarrow V$ defines embeddings $V_{n \lambda} \hookrightarrow S_{\sigma}^{n} V$ for all $n \geq 2$. In particular, the algebra $S_{\sigma}(V)$ is infinite-dimensional.

Proof. By definition, an embedding $V_{\lambda} \hookrightarrow V$ is equivalent to the existence of a highest weight vector $v_{\lambda}$ in the weight space $V(\lambda)$. Then $V_{\lambda} \cong U_{q}(\mathfrak{g})\left(v_{\lambda}\right)$.

Denote by $v_{n \lambda}=v_{\lambda} \otimes v_{\lambda} \otimes \cdots \otimes v_{\lambda} \in V^{\otimes n}$. First, show that $v_{2 \lambda} \in S_{\sigma}^{2} V$. Indeed,

$$
\mathcal{R}_{V \otimes V}\left(v_{2 \lambda}\right)=\mathcal{R}_{V \otimes V}\left(v_{\lambda} \otimes v_{\lambda}\right)=q^{(\lambda \mid \lambda)} v_{\lambda} \otimes v_{\lambda}=D\left(v_{\lambda} \otimes v_{\lambda}\right) .
$$

Therefore, $\sigma_{V \otimes V}\left(v_{2 \lambda}\right)=v_{2 \lambda}$ and $v_{2 \lambda} \in S_{\sigma}^{2} V$. This implies that $\sigma_{i, i+1}\left(v_{n \lambda}\right)=v_{n \lambda}$ for $i=1, \ldots, n-1$. Hence $v_{n \lambda} \in S_{\sigma}^{n} V$ and $V_{n \lambda} \hookrightarrow S_{\sigma}^{n} V$. Proposition 2.13 is proved.

2.2. Braided algebras as deformations of Poisson structures. In this section we study "classical" (at $q=1$ ) analogues of the braided symmetric and exterior algebras and present the braided objects as $q$-deformations of the classical ones. More precisely, using the fact that the "classical limit" of each braided algebra is a commutative $\mathbb{C}$-(super)algebra, we will investigate the (super-)Poisson structures emerging as "classical limits" of the (super-)commutators in the braided algebras (Theorem 2.21 below).

Definition 2.14. Given a $\mathbb{Z}_{2}$-graded algebra $A=A_{\overline{0}} \oplus A_{\overline{1}}$, (i.e., $A_{\varepsilon} \cdot A_{\delta} \subset A_{\varepsilon+\delta}$ for any $\left.\varepsilon, \delta \in \mathbb{Z}_{2}=\mathbb{Z} /(2)=\{\overline{0}, \overline{1}\}\right)$, we say that $A$ is a commutative superalgebra if

$$
b a=(-1)^{\varepsilon \delta} a b
$$

for any $a \in A_{\varepsilon}$ and $b \in A_{\delta}, \varepsilon, \delta \in \mathbb{Z}_{2}=\{\overline{0}, \overline{1}\}$.

Definition 2.15. A bracketed superalgebra is a pair $(A,\{\cdot, \cdot\})$ where $A$ is a commutative superalgebra and a bilinear map $\{\cdot, \cdot\}: A \times A \rightarrow A$ such that $\left\{A_{\varepsilon}, A_{\delta}\right\} \subset A_{\varepsilon+\delta}$ for any $\varepsilon, \delta \in \mathbb{Z}_{2}$ and the following identities hold:

(i) super-anti-commutativity:

$$
\{a, b\}+(-1)^{\varepsilon \delta}\{b, a\}=0
$$

for any $a \in A_{\varepsilon}, b \in A_{\delta}$, 
(ii) the super-Leibniz rule

$$
\{a, b c\}=\{a, b\} c+(-1)^{\varepsilon \delta} b\{a, c\}
$$

for any $a \in A_{\varepsilon}, b \in A_{\delta}, c \in A_{\gamma}$.

If, in addition, the bracket satisfies

(iii) the super-Jacobi identity:

$$
(-1)^{\varepsilon \gamma}\{a,\{b, c\}\}+(-1)^{\gamma \delta}\{c,\{a, b\}\}+(-1)^{\delta \varepsilon}\{b,\{c, a\}\}=0
$$

for any $a \in A_{\varepsilon}, b \in A_{\delta}, c \in A_{\gamma}$, then we will refer to $A$ as a Poisson superalgebra and will refer to $\{\cdot, \cdot\}$ as super-Poisson bracket.

We define the tensor product $A \otimes B$ of two bracketed superalgebras $A$ and $B$ to be the usual tensor product in the category of superalgebras with bracket $\{\cdot, \cdot\}$ :

$$
\left\{a \otimes b, a^{\prime} \otimes b^{\prime}\right\}=(-1)^{\varepsilon^{\prime} \delta} a a^{\prime} \otimes\left\{b, b^{\prime}\right\}+(-1)^{\delta \varepsilon^{\prime}+\delta \delta^{\prime}}\left\{a, a^{\prime}\right\} \otimes b b^{\prime},
$$

for all $a \in A, b^{\prime} \in B, a^{\prime} \in A_{\varepsilon^{\prime}}, b \in B_{\delta}$.

For each reductive Lie algebra $\mathfrak{g}$ denote by $\overline{\mathcal{O}}_{f}=\overline{\mathcal{O}}_{f}(\mathfrak{g})$ the category of finitedimensional $\mathfrak{g}$-modules $\bar{V}$ such that the center $Z(\mathfrak{g})$ acts semi-simply on $\bar{V}$.

Recall that the classical $r$-matrix of $\mathfrak{g}$ is a $\mathfrak{g}$-invariant element $r \in \mathfrak{g} \otimes \mathfrak{g}$ satisfying the classical Yang-Baxter equation $[[r, r]]=0$ and $r+\tau(r)=c$, where $\tau$ is the permutation of factors and $c \in S^{2}(\mathfrak{g})$ is the Casimir element. Denote by $r^{-} \in \mathfrak{g} \wedge \mathfrak{g}$ the anti-symmetrization of $r$, i.e.,

$$
r^{-}=\frac{1}{2}(r-\tau(r)) .
$$

Note that $r^{-}=\sum_{\alpha} E_{\alpha} \otimes F_{\alpha}-F_{\alpha} \otimes F_{\alpha}$, where the summation is over all positive roots of $\mathfrak{g}=\mathfrak{n}_{-} \oplus \mathfrak{h} \oplus \mathfrak{n}_{+},\left\{E_{\alpha}\right\}$ is a basis for $\mathfrak{n}_{+}$, and $\left\{F_{\alpha}\right\}$ is the basis in $\mathfrak{n}_{+}$dual to the former one with respect to the Killing form.

Example 2.16. Recall that $g l_{2}(\mathbb{C})$ has a standard basis of matrix units $E, F, H_{1}, H_{2}$ and $r=2 E \otimes F+H_{1} \otimes H_{1}+H_{2} \otimes H_{2}$. Therefore, $r^{-}=\frac{1}{2}(r-\tau(r))=E \otimes F-F \otimes E=$ $E \wedge F$.

The following fact is obvious.

Lemma 2.17. (a) Let $\bar{V}$ be any finite-dimensional $\mathfrak{g}$-module. Then the element $r^{-}$considered as a $\mathfrak{g}$-equivariant operator $r^{-}: \bar{V} \otimes \bar{V} \rightarrow \bar{V} \otimes \bar{V}$ satisfies

$$
r^{-}\left(\Lambda^{2}(\bar{V})\right) \subset S^{2}(\bar{V}), r^{-}\left(S^{2}(\bar{V})\right) \subset \Lambda^{2}(\bar{V}) .
$$

(b) The symmetric algebra $S(\bar{V})$ considered as an even superalgebra (i.e., $S(\bar{V})_{\overline{0}}$ $=S(\bar{V})$ ) has a unique homogeneous bracket $\{\cdot, \cdot\}_{+}: S(\bar{V}) \times S(\bar{V}) \rightarrow S(\bar{V})$ such that

$$
\{u, v\}_{+}=r^{-}(u \cdot v)
$$

for any $u, v \in \bar{V}$.

(c) The symmetric algebra $\Lambda(\bar{V})$ considered as a superalgebra with odd $\bar{V}$ has a unique homogeneous super-bracket $\{\cdot, \cdot\}_{-}: \Lambda(\bar{V}) \times \Lambda(\bar{V}) \rightarrow \Lambda(\bar{V})$ such that

$$
\{u, v\}_{-}=r^{-}(u \wedge v)
$$

for any $u, v \in \bar{V}$. 
For any bracketed superalgebra define the super-Jacobian map $J: A \times A \times A \rightarrow A$ by:

$$
J(a, b, c)=(-1)^{\varepsilon \gamma}\{a,\{b, c\}\}+(-1)^{\gamma \delta}\{c,\{a, b\}\}+(-1)^{\delta \varepsilon}\{b,\{c, a\}\}
$$

for any $a \in A_{\varepsilon}, b \in A_{\delta}, c \in A_{\gamma}$.

Define the ideals $I_{+}(\bar{V})$ and $I_{-}(\bar{V})$ in $S(\bar{V})$ and $\Lambda(\bar{V})$ respectively by:

$$
I_{+}(\bar{V}):=J_{+}(\bar{V}, \bar{V}, \bar{V}) \cdot S(\bar{V}), I_{-}(\bar{V}):=J_{-}(\bar{V}, \bar{V}, \bar{V}) \wedge \Lambda(\bar{V})
$$

where $J_{+}$and $J_{-}$are respective Jacobian maps as in (2.6). Then define algebras

$$
\overline{S(\bar{V})}:=S(\bar{V}) / I_{+}(\bar{V}), \overline{\Lambda(\bar{V})}:=\Lambda(\bar{V}) / I_{-}(\bar{V}) \text {. }
$$

Theorem 2.18. For each $\bar{V}$ in $\overline{\mathcal{O}}_{f}$ we have (using the Sweedler-like notation $r^{-}=$ $\left.r_{(1)}^{-} \otimes r_{(2)}^{-}\right):$

(a) The brackets $\{\cdot, \cdot\}_{+}$and $\{\cdot, \cdot\}_{-}$are given by:

$$
\{a, b\}_{+}=r_{(1)}^{-}(a) \cdot r_{(2)}^{-}(b)
$$

for each $a, b \in S(\bar{V})$.

$$
\{a, b\}_{-}=r_{(1)}^{-}(a) \wedge r_{(2)}^{-}(b)
$$

for each $a, b \in \Lambda(\bar{V})$.

(b) The homogeneous bracketed (super)algebras $\overline{S(\bar{V})}$ and $\overline{\Lambda(\bar{V})}$ are Poisson with the (super)Poisson brackets given by the above formulas (2.8) and (2.9).

We will prove Theorem 2.18 in Section 3

Example 2.19. Recall from Example 2.16 that for $\mathfrak{g}=g l_{2}(\mathbb{C})$ we have $r^{-}=$ $E \wedge F=E \otimes F-F \otimes E$. Theorem 2.18 guarantees that for any $\bar{V}$ in $\overline{\mathcal{O}}_{f}$ one has:

$$
\{a, b\}_{+}=E(a) F(b)-F(a) E(b)
$$

for all $a, b \in S(\bar{V})$ and

$$
\{a, b\}_{-}=E(a) \wedge F(b)-F(a) \wedge E(b)
$$

for all $a, b \in \Lambda(\bar{V})$.

Corollary 2.20 (from the proof of Theorem 2.18). The Poisson (super)algebras $\overline{S(\bar{V})}=S(\bar{V}) / I_{+}(\bar{V})$ and $\overline{\Lambda(\bar{V})}=\Lambda(\bar{V}) / I_{-}(\bar{V})$ are Poisson closures of $S(\bar{V})$ and $\Lambda(\bar{V})$ respectively.

The following is the second main result of this section.

Main Theorem 2.21. Let $V$ be an object of $\mathcal{O}_{f}$ and let $\bar{V}$ in $\overline{\mathcal{O}}_{f}$ be the classical limit of $V$. Then:

(a) The braided symmetric algebra $S_{\sigma}(V)$ is a flat q-deformation of a certain Poisson quotient of the Poisson algebra $\overline{S(\bar{V})}$. In particular, $\operatorname{dim}_{\mathbb{C}(q)} S_{\sigma}(V)_{n} \leq$ $\operatorname{dim}_{\mathbb{C}} \overline{S(\bar{V})_{n}}$.

(b) The braided exterior algebra $\Lambda_{\sigma}(V)$ is a flat q-deformation of a certain Poisson quotient of the Poisson superalgebra $\overline{\Lambda(\bar{V})}$. E.g., $\operatorname{dim}_{\mathbb{C}(q)} \Lambda_{\sigma}(V)_{n} \leq \operatorname{dim}_{\mathbb{C}} \overline{\Lambda(\bar{V})_{n}}$.

We will prove Theorem 2.21 in Section 3 .

Now we can post two natural problems. 
Problem 2.22. Describe all those $U_{q}(\mathfrak{g})$-modules in $\bar{V}$ for which $S_{\sigma}(V)$ is a flat deformation of $\overline{S(\bar{V})}$.

Even though we have only a little evidence, we would expect that each irreducible $V=V_{\lambda}$ in $\mathcal{O}_{f}$ solves the problem.

Since $\overline{S_{\sigma}(\bar{V})}$ is a quotient algebra of the symmetric algebra $S(\bar{V})$, we can define an affine scheme $X(\bar{V})=\operatorname{spec} \overline{S_{\sigma}(\bar{V})}$ in $\bar{V}^{*}$.

Problem 2.23. For each $V \in O b\left(\mathcal{O}_{f}\right)$ study the scheme $X(\bar{V})$. In particular, describe all objects $V$ for which the scheme $X(\bar{V})$ is a variety.

Clearly, if $V$ is flat (see Section 2.3), then $X(\bar{V})=\bar{V}^{*}$ is a variety. It would be interesting to see what happens with $X(\bar{V}) \neq \bar{V}$, i.e., $S_{\sigma}(V)$ is not a flat deformation of $S(\bar{V})$ (see Sections 2.3 and 2.5 below).

To study the scheme $X(\bar{V})$ it would be helpful to estimate the growth of braided symmetric algebras. We will conclude the section with a few corollaries from Theorem 2.21 and one conjecture concerning the Hilbert series of braided algebras.

Definition 2.24. For each $U=\bigoplus_{n \geq 0} U_{n}$ in the category $\mathcal{O}_{g r, f}$ define the Hilbert series $h(U, t) \in \mathbb{Z}[[t]]$ by:

$$
h(U, t)=\sum_{i=0}^{\infty} \operatorname{dim}\left(U_{i}\right) t^{i} .
$$

According to Theorem 2.21(b), $\Lambda_{\sigma}(V)$ is bounded from above (as a vector space) by the ordinary exterior algebra $\Lambda(V)$. Therefore, the Hilbert series $h\left(\Lambda_{\sigma}(V), t\right)$ is a polynomial in $t$ of degree at most $\operatorname{dim}(V)$.

Corollary 2.25. For any $V \in O b\left(\mathcal{O}_{f}\right)$ the Hilbert series $h\left(S_{\sigma}(V), t\right)$ is a rational function in $t$ of the form $\frac{p(t)}{(1-t)^{d}}$, where $p(t)$ is a polynomial and $d \leq \operatorname{dim} V$.

Proof. Theorem 2.21 asserts that $S_{\sigma}(V)$ is a flat deformation of a certain quotient algebra of the symmetric algebra $S(\bar{V})$, where $\bar{V}$ is "the classical limit" of $V$. Therefore, the Hilbert series $h\left(S_{\sigma}(V), t\right)$ equals the Hilbert series of a quotient of algebra of $S(\bar{V})$ by a certain homogeneous ideal $\bar{J}$. It is well-known that $h(S(\bar{V}))=$ $\frac{1}{(1-t)^{\operatorname{dim} V}}$ and well-known that the Hilbert series of $S(\bar{V}) / \bar{J}$ can be expressed as a rational function in $t$ with the denominator $(1-t)^{d}$. This proves the corollary.

We conclude the section with a conjectural observation about "numerical Koszul duality" between the braided symmetric and exterior algebras.

Conjecture 2.26. For each $\lambda \in P_{+}$we have

$$
h\left(S_{\sigma}\left(V_{\lambda}\right), t\right) \cdot h\left(\Lambda_{\sigma}\left(V_{\lambda}^{*}\right),-t\right)=1+O\left(t^{4}\right) .
$$

Or, equivalently, $\operatorname{dim} S_{\sigma}^{3} V_{\lambda}-\operatorname{dim} \Lambda_{\sigma}^{3} V_{\lambda}^{*}=\left(\operatorname{dim} V_{\lambda}\right)^{2}$.

Indeed, if $S_{\sigma}\left(V_{\lambda}\right)$ is Koszul, then the conjecture obviously holds without the term $O\left(t^{4}\right)$. For non-Koszul $S_{\sigma}\left(V_{\lambda}\right)$, we have verified the conjecture numerically in several cases (see, e.g., Corollary 2.36 below). Moreover, in the non-Koszul case we expect that the term $O\left(t^{4}\right)$ will never vanish. 
2.3. Flat modules. We view $S_{\sigma}(V)$ and $\Lambda_{\sigma}(V)$ as deformations of the quadratic algebras $S(V)$ and $\Lambda(V)$, respectively. Theorem 2.21 (taken in conjunction with Proposition 2.11(b)) implies that

$$
\operatorname{dim} S_{\sigma}^{n} V=\operatorname{dim} S_{\sigma}(V)_{n} \leq\left(\begin{array}{c}
\operatorname{dim} V+n-1 \\
n
\end{array}\right)
$$

for all $n$.

Therefore, we come to the following definition.

Definition 2.27. A finite dimensional $U_{q}(\mathfrak{g})$-module is flat, if and only if

$$
\operatorname{dim} S_{\sigma}^{n} V=\left(\begin{array}{c}
\operatorname{dim} V+n-1 \\
n
\end{array}\right)
$$

for all $n \geq 0$; i.e., the braided symmetric power $S_{\sigma}^{n} V$ is isomorphic (as a vector space) to the ordinary symmetric power $S^{n} V$.

By definition, $\operatorname{dim} S_{\sigma}^{n} V=(\underset{n}{\operatorname{dim} V+1})$ for $n=0,1,2$. To determine whether $V$ is flat it is sufficient to determine the flatness of $S_{\sigma}^{3} V$ due to the following result.

Proposition 2.28. A module $V$ in $\mathcal{O}_{f}$ is flat if and only if

$$
\operatorname{dim} S_{\sigma}^{3} V=\operatorname{dim} S^{3} V=\left(\begin{array}{c}
\operatorname{dim} V+2 \\
3
\end{array}\right) .
$$

Moreover, if $S_{\sigma}(V)$ is flat, then it is Koszul.

Proof. Follows from Drinfeld's result ([14, Theorem 1]) which asserts that if $A=$ $T(V) /\left\langle I_{2}\right\rangle$ is a quadratic algebra over $\mathbb{C}(h)$ such that $I_{2} \subset V \otimes V$ is a flat $h$ deformation of the exterior square $\Lambda^{2} V$ (i.e., $I_{2}$ has a basis of the form $e_{i} \otimes e_{j}-$ $e_{j} \otimes e_{i}-h \sum_{k, l} c_{i j}^{k l} e_{k} \otimes e_{l}$ for $1 \leq i<j \leq \operatorname{dim} V$, where all $\left.c_{i j}^{k, l} \in \mathbb{C}[[h]]\right)$ and $\operatorname{dim} A_{3}=\operatorname{dim} S^{3} V$, then $\operatorname{dim} A_{n}=\operatorname{dim} S^{n} V$ for all $n$. The Koszulity of such an $A$ follows because $A$ has a PBW-basis. The proposition is proved.

The above result is a first step toward solving the following problem.

Problem 2.29. Classify all flat $U_{q}(\mathfrak{g})$-modules $V$ in $\mathcal{O}_{f}$.

We expect that for each semisimple Lie algebra $\mathfrak{g}$ there are only finitely many flat simple modules $V_{\lambda}$. Next, we compute a lower bound for dimension of each $S_{\sigma}^{3} V_{\lambda}$. We will use the notation

$$
V^{\mu}
$$

for the space of highest weight vectors of weight $\mu$ in a module $V$ in $\mathcal{O}_{f}$. For $\lambda, \mu, \nu \in P_{+}$denote $c_{\lambda, \mu}^{\nu}=\operatorname{dim}\left(V_{\lambda} \otimes V_{\mu}\right)^{\nu}$, i.e., $c_{\lambda, \mu}^{\nu}$ is the tensor product multiplicity. Also, for any $\lambda, \mu \in P_{+}$denote $c_{\lambda ; \mu}^{-}=\operatorname{dim}\left(S_{\sigma}^{2} V_{\lambda}\right)^{\mu}$ and $c_{\lambda ; \mu}^{+}=\operatorname{dim}\left(\Lambda_{\sigma}^{2} V_{\lambda}\right)^{\mu}$, so that $c_{\lambda ; \mu}^{-}+c_{\lambda ; \mu}^{+}=c_{\lambda, \lambda}^{\mu}$. Ultimately, define:

$$
d_{\lambda}^{\mu}:=\sum_{\nu \in P_{+}}\left(c_{\lambda ; \nu}^{+}-c_{\lambda ; \nu}^{-}\right) c_{\nu, \lambda}^{\mu} .
$$

Lemma 2.30. For each $\lambda \in P_{+}$we have

$$
\operatorname{dim} S_{\sigma}^{3} V_{\lambda} \geq \sum_{\mu \in P_{+}} \max \left(d_{\lambda}^{\mu}, 0\right) \cdot \operatorname{dim} V_{\mu} .
$$


Proof. By definition of $S_{\sigma}^{3} V_{\lambda}$, one has $\left(S_{\sigma}^{3} V_{\lambda}\right)^{\mu}=\left(S_{\sigma}^{2} V_{\lambda} \otimes V_{\lambda}\right)^{\mu} \cap\left(V_{\lambda} \otimes S_{\sigma}^{2} V_{\lambda}\right)^{\mu}$. Therefore, we obtain the inequality:

$$
\begin{gathered}
\operatorname{dim}\left(S_{\sigma}^{3} V_{\lambda}\right)^{\mu} \geq \operatorname{dim}\left(S_{\sigma}^{2} V_{\lambda} \otimes V_{\lambda}\right)^{\mu}+\operatorname{dim}\left(V_{\lambda} \otimes S_{\sigma}^{2} V_{\lambda}\right)^{\mu}-\operatorname{dim}\left(V_{\lambda} \otimes V_{\lambda} \otimes V_{\lambda}\right)^{\mu} \\
=\operatorname{dim}\left(S_{\sigma}^{2} V_{\lambda} \otimes V_{\lambda}\right)^{\mu}-\operatorname{dim}\left(V_{\lambda} \otimes \Lambda_{\sigma}^{2} V_{\lambda}\right)^{\mu}=d_{\lambda}^{\mu} .
\end{gathered}
$$

Taking into account that $\operatorname{dim} V=\bigoplus_{\mu \in P_{+}} \operatorname{dim} V^{\mu} \cdot \operatorname{dim} V_{\mu}$, we obtain (2.12).

Using this result, we obtain the following sufficient criterion of flatness.

Proposition 2.31. Assume that

$$
\sum_{\mu \in P_{+}} \max \left(d_{\lambda}^{\mu}, 0\right) \cdot \operatorname{dim} V_{\mu}=\left(\begin{array}{c}
\operatorname{dim} V_{\lambda}+2 \\
3
\end{array}\right)
$$

for some $\lambda \in P_{+}$. Then the simple $U_{q}(\mathfrak{g})$-module $V_{\lambda}$ is flat.

Proof. Follows from (2.12) and Proposition 2.28

Based on the examples below (and on Corollary 2.36 below), we expect that this sufficient criterion of flatness is also necessary. In what follows we will provide some examples of flat modules $V_{\lambda}$, each of which satisfies (2.13). Along these lines we expect the "orthogonality" of the symmetric and exterior cubes: if $\left(S_{\sigma}^{3} V_{\lambda}\right)^{\mu} \neq 0$ (in the notation of (2.11)), then $\left(\Lambda_{\sigma}^{3} V_{\lambda}\right)^{\mu}=0$ and vice versa.

2.4. Examples of flat modules. Recall that for $U_{q}\left(g l_{d}(\mathbb{C})\right)$-modules the dominant weights are non-increasing $d$-tuples of integers $\lambda=\left(\lambda_{1} \geq \lambda_{2} \geq \cdots \lambda_{d}\right)$. The $d$-tuple $\omega_{i}=(1,1, \ldots, 1,0, \ldots, 0)$ having $i$ ones and $d-i$ zeros is referred to as the $i$-th fundamental weight. In particular, $V_{\omega_{1}} \cong(\mathbb{C}(q))^{d}$ is the standard $U_{q}\left(g l_{d}(\mathbb{C})\right)$ module.

Lemma 2.32. The standard $U_{q}\left(g l_{d}(\mathbb{C})\right)$-module $V_{\omega_{1}}$ is flat. More precisely,

(a) Each graded component $S_{\sigma}\left(V_{\omega_{1}}\right)_{n}$ is irreducible and isomorphic to $V_{n \omega_{1}}$.

(b) As an algebra $S_{\sigma}\left(V_{\omega_{1}}\right)$ is isomorphic to $\mathbb{C}_{q}\left[x_{1}, \ldots, x_{d}\right]$, q-polynomial algebra generated by $x_{1}, \ldots, x_{n}$ subject to the relations $x_{j} x_{i}=q x_{i} x_{j}$ for $1 \leq i<j \leq d$.

Proof. Prove (a). It suffices to show that $S_{\sigma}^{n} V_{\omega_{1}} \cong V_{n \omega_{1}}$. Indeed, by Proposition 2.13 with $\lambda=\omega_{1}$ and $V=V_{\omega_{1}}$, one has an injective homomorphism $V_{n \omega_{1}} \hookrightarrow S_{\sigma}^{n} V_{\omega_{1}}$. Also, by the dimension count, this homomorphism is surjective.

Prove (b) now. Let us choose a standard weight basis $x_{1}, \ldots, x_{d}$ in $V_{\omega_{1}}$. That is, $E_{i}\left(x_{j}\right)=\delta_{i, j-1} x_{j-1}$ and $F_{i}\left(x_{j}\right)=\delta_{i, j} v_{j+1}, K_{\lambda}\left(x_{j}\right)=q^{\lambda_{j}} x_{j}$. The formula (4.4) implies that

$$
\Lambda_{\sigma}^{2} V_{\omega_{1}}=\left\langle x_{j} \otimes x_{i}-q x_{i} \otimes x_{j} \mid i<j\right\rangle, S_{\sigma}^{2} V_{\omega_{1}}=\left\langle x_{i} \otimes x_{j}+q x_{j} \otimes x_{i} \mid i \leq j\right\rangle,
$$

where $\langle\cdot\rangle$ denotes the $\mathbb{C}(q)$-linear span. Therefore, the quotient algebra $S_{\sigma}\left(V_{\omega_{1}}\right)=$ $T\left(V_{\omega_{1}}\right) /\left\langle\Lambda_{\sigma}^{2} V_{\omega_{1}}\right\rangle$ is naturally isomorphic to $\mathbb{C}_{q}\left[x_{1}, \ldots, x_{d}\right]$. This proves (b). The proposition is proved.

A more general example is the space $M_{d \times k}$ of all $d \times k$-matrices over $\mathbb{C}(q)$ regarded as a $U_{q}\left(g l_{d}(\mathbb{C}) \times g l_{k}(\mathbb{C})\right)$-module. Recall that $\mathbb{C}_{q}\left[M_{d \times k}\right]$ is the algebra of quantum regular functions on the space $d \times k$-matrices (for the reader's convenience, the definition and main properties of $\mathbb{C}_{q}\left[M_{d \times k}\right]$ are given in the Appendix).

Proposition 2.33. For any $d, k \geq 1$ one has an isomorphism of algebras in $\mathcal{O}_{g r, f}$ : $S_{\sigma}\left(M_{d \times k}\right) \cong \mathbb{C}_{q}\left[M_{d \times k}\right]$. In particular, the $U_{q}\left(g l_{d}(\mathbb{C}) \times g l_{k}(\mathbb{C})\right)$-module $M_{d, k}$ is flat. 
Proof. Let us identify the weight lattice $P^{+}$for $U_{q}\left(g l_{d}(\mathbb{C}) \times g l_{k}(\mathbb{C})\right)$ with $\mathbb{Z}^{d} \times \mathbb{Z}^{k}$. Let $V_{1}:=V_{\left(\omega_{1}, 0\right)}, V_{2}:=V_{\left(0, \omega_{1}\right)}$ so that

$$
M_{d \times k} \cong V_{\left(\omega_{1}, \omega_{1}\right)} \cong V_{1} \otimes V_{2}
$$

as a $U_{q}\left(g l_{d}(\mathbb{C}) \times g l_{k}(\mathbb{C})\right)$-module. By definition, both braiding operators $\mathcal{R}_{V_{1}, V_{2}}$ : $V_{1} \otimes V_{2} \rightarrow V_{2} \otimes V_{1}$ and $\mathcal{R}_{V_{2}, V_{1}}: V_{2} \otimes V_{1} \rightarrow V_{1} \otimes V_{2}$ are merely permutations of factors. Therefore,

$$
\mathcal{R}_{M_{d \times k}, M_{d \times k}}=\tau_{23} \circ\left(\mathcal{R}_{V_{1}, V_{1}} \otimes \mathcal{R}_{V_{2}, V_{2}}\right) \circ \tau_{23}
$$

where $\tau_{23}$ is the permutation of two middle factors in the tensor product of four modules. In its turn, it implies that

$$
\Lambda_{\sigma}^{2} M_{d \times k}=\tau_{23}\left(S_{\sigma}^{2} V_{1} \otimes \Lambda_{\sigma}^{2} V_{2} \oplus \Lambda_{\sigma}^{2} V_{1} \otimes S_{\sigma}^{2} V_{2}\right) .
$$

Then using the standard basis $x_{1}, x_{2}, \ldots, x_{d}$ for $V_{1}$ and the standard $x_{1}^{\prime}, x_{2}^{\prime}, \ldots, x_{k}^{\prime}$ for $V_{2}$, the formula (2.14), and the notation $x_{i j}:=x_{i} \otimes x_{j}^{\prime}$, we see that $\Lambda_{\sigma}^{2} M_{d \times k}$ is freely spanned by the elements of the form

$x_{i, j^{\prime}} \otimes x_{i, j}-q x_{i, j} \otimes x_{i, j^{\prime}}, x_{i, j^{\prime}} \otimes x_{i^{\prime}, j}+q x_{i^{\prime}, j^{\prime}} \otimes x_{i, j}-q x_{i, j} \otimes x_{i^{\prime}, j^{\prime}}-q^{2} x_{i^{\prime}, j} \otimes x_{i, j^{\prime}}$, $x_{i^{\prime}, j} \otimes x_{i, j}-q x_{i, j} \otimes x_{i^{\prime}, j}, x_{i^{\prime}, j^{\prime}} \otimes x_{i, j}+q x_{i^{\prime}, j^{\prime}} \otimes x_{i, j}-q x_{i, j} \otimes x_{i^{\prime}, j^{\prime}}-q^{2} x_{i, j^{\prime}} \otimes x_{i^{\prime}, j}$ for $1 \leq i<i^{\prime} \leq d, 1 \leq j<j^{\prime} \leq d$. Therefore, the quotient algebra $S_{\sigma}\left(M_{d \times k}\right)=$ $T\left(M_{d \times k}\right) /\left\langle\Lambda_{\sigma}^{2} M_{d \times k}\right\rangle$ is naturally isomorphic to $\mathbb{C}_{q}\left[M_{d \times k}\right]$.

The proposition is proved.

2.5. Braided symmetric and exterior powers of simple $U_{q}\left(g l_{2}(\mathbb{C})\right)$-modules. Recall that irreducible $U_{q}\left(g l_{2}(\mathbb{C})\right)$-modules $V_{\lambda}$ in $\mathcal{O}_{f}$ are labeled by the pairs $\lambda=$ $\left(\lambda_{1} \geq \lambda_{2}\right)$ of integers and $\operatorname{dim} V_{\lambda}=\lambda_{1}-\lambda_{2}+1$.

In what follows we will abbreviate $V_{\ell}:=V_{(\ell, 0)}$ for $\ell \in \mathbb{Z}_{\geq 0}$. Note that in terms of braided symmetric powers, $V_{\ell} \cong S_{\sigma}^{\ell} V_{1}$ for each $\ell \geq 0$.

It is easy to see that $\left(V_{\left(\lambda_{1}, \lambda_{2}\right)}\right)^{\otimes n} \cong V_{\left(n \lambda_{2}, n \lambda_{2}\right)} \otimes\left(V_{\lambda_{1}-\lambda_{2}}\right)^{\otimes n}$ and

$$
\Lambda_{\sigma}^{n} V_{\left(\lambda_{1}, \lambda_{2}\right)} \cong V_{\left(n \lambda_{2}, n \lambda_{2}\right)} \otimes \Lambda_{\sigma}^{n} V_{\lambda_{1}-\lambda_{2}}, S_{\sigma}^{n} V_{\left(\lambda_{1}, \lambda_{2}\right)} \cong V_{\left(n \lambda_{2}, n \lambda_{2}\right)} \otimes S_{\sigma}^{n} V_{\lambda_{1}-\lambda_{2}}
$$

for any $\lambda_{1} \geq \lambda_{2}$ and $n \geq 0$.

The following is our first main result for $U_{q}\left(g l_{2}(\mathbb{C})\right)$-modules.

Main Theorem 2.34. For each $\ell \geq 3$ and $n \geq 4$ we have $\Lambda^{n} V_{\ell}=0$.

We will proof Theorem 2.34 in Section 3 ,

Our second main result gives a complete description of the braided exterior and the braided symmetric cube of each $V_{\ell}$.

Main Theorem 2.35. For each $\ell \geq 0$ and each $n \geq 3$ one has:

$$
\begin{gathered}
S_{\sigma}^{3} V_{\ell} \cong \begin{cases}\bigoplus_{0 \leq i \leq \frac{\ell-1}{2}} V_{(3 \ell-2 i, 2 i)} & \text { if } \ell \text { is odd }, \\
\bigoplus_{0 \leq i \leq \frac{3 \ell}{4}} V_{(3 \ell-2 i, 2 i)} & \text { if } \ell \text { is even, }\end{cases} \\
\Lambda_{\sigma}^{3} V_{\ell} \cong \begin{cases}0 & \text { if } \ell \text { is odd }, \\
\bigoplus_{\frac{\ell}{2} \leq i \leq \frac{3 \ell-2}{4}} V_{(3 \ell-2 i-1,2 i+1)} & \text { if } \ell \text { is even. }\end{cases}
\end{gathered}
$$

We will proof Theorem 2.35 in Section 3 The following is an immediate corollary of Theorem 2.35. 
Corollary 2.36. For each $\ell \geq 0$ we have:

$$
\operatorname{dim} \Lambda_{\sigma}^{3} V_{\ell}=\delta_{\ell} \cdot\left(\begin{array}{c}
\frac{\ell}{2}+1 \\
2
\end{array}\right), \operatorname{dim} S_{\sigma}^{3} V_{\ell}=(\ell+1)^{2}+\delta_{\ell} \cdot\left(\begin{array}{c}
\frac{\ell}{2}+1 \\
2
\end{array}\right),
$$

where $\delta_{\ell}=0$ if $\ell$ is odd and $\delta_{\ell}=1$ if $\ell$ is even. In particular, $V_{\ell}$ is flat if and only if $\ell \in\{0,1,2\}$.

Based on Theorem 2.35, we propose the following conjectural description of the higher braided symmetric powers of $V_{\lambda}$ for any $\lambda$.

Conjecture 2.37. For any $\ell \geq 0$ and any $n \geq 4$ one has:

$$
S_{\sigma}^{n} V_{\ell} \cong \begin{cases}\bigoplus_{0 \leq i \leq \frac{\ell-1}{2}} V_{(n \ell-2 i, 2 i)} & \text { if } \ell \text { is odd } \\ \bigoplus_{0 \leq i \leq \frac{n \ell}{4}} V_{(n \ell-2 i, 2 i)} & \text { if } \ell \text { is even }\end{cases}
$$

In particular, $S_{\sigma}^{n} V_{\ell}$ is multiplicity-free and we have for $n \geq 4$ :

$$
\operatorname{dim} S_{\sigma}^{n} V_{\ell}= \begin{cases}\frac{(\ell+1)(\ell(n-1)+2)}{2} & \text { if } \ell \text { is odd }, \\ \left(\frac{n \ell}{2}+2\right) & \text { if } \ell \text { is even. }\end{cases}
$$

So far we have collected some partial evidence for Conjecture 2.37, obtained from analyzing the Poisson closure $\overline{S\left(\bar{V}_{\ell}\right)}$ of the bracketed superalgebra $\left(S\left(\bar{V}_{\ell}\right),\{\cdot, \cdot\}\right)$ (see (2.7)) which serves as an upper bound for the braided symmetric algebra $S_{\sigma}\left(V_{\ell}\right)$ - we expect that the upper bound is achieved for all $\ell$. For instance, using the computer algebra system SINGULAR ([15]), we have verified that for $\ell=3,4,5,6$ the dimension of each component $\overline{S\left(\bar{V}_{\ell}\right)}$ equals the right hand side of (2.19). Taken in conjunction with Proposition2.11 and Theorem 2.21(a), this gives a correct upper bound on $\operatorname{dim} S_{\sigma}^{n} V_{\ell}$.

Another piece of evidence comes from Proposition 2.13 which implies that $S_{\sigma}^{n} V_{\ell}$ always contains a unique copy of the simple $g l_{2}(\mathbb{C})$-module $\bar{V}_{n \ell}$. We also verified

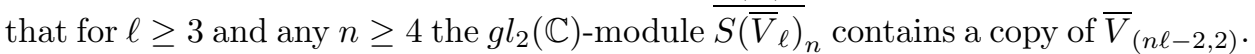

Using (2.17) we now describe all those modules $V_{\ell}$ whose braided symmetric algebra is Koszul.

Corollary 2.38. The braided symmetric algebra $S_{\sigma}\left(V_{\ell}\right)$ is Koszul if and only if $\ell \leq 2$.

Proof. For $\ell \leq 2$ the module $V_{\ell}$ is flat and the assertion follows from Proposition 2.28. Now let $\ell \geq 3$. If $S_{\sigma}\left(V_{\ell}\right)$ was Koszul, then the Hilbert series $h\left(S_{\sigma}\left(V_{\ell}\right), t\right)$ (see Definition 2.24) would satisfy:

$$
h\left(S_{\sigma}\left(V_{\ell}\right), t\right)=\frac{1}{h\left(S_{\sigma}\left(V_{\ell}\right)^{!},-t\right)} .
$$

Since $S_{\sigma}\left(V_{\ell}\right)^{!} \cong \Lambda_{\sigma}\left(\left(V_{\ell}\right)^{*}\right)$ by Proposition 2.11(c), $\Lambda_{\sigma}\left(\left(V_{\ell}\right)^{*}\right)$ and $\left.\Lambda_{\sigma}\left(V_{\ell}\right)\right)$ are isomorphic as graded vector spaces, and the Koszulity of $S_{\sigma}\left(V_{\ell}\right)$ would imply that all the coefficients in the power series expansion for the rational function $\frac{1}{h\left(\Lambda_{\sigma}\left(V_{\ell}\right),-t\right)}$ are non-negative.

According to Corollary 2.36, one has

$$
h\left(\Lambda_{\sigma}\left(V_{\ell}\right),-t\right)=1-(\ell+1) t+\frac{\ell(\ell+1)}{2} t^{2}-\delta_{\ell} \cdot \frac{\ell(\ell+2)}{8} t^{3} .
$$


Therefore,

$$
\frac{1}{h\left(\Lambda_{\sigma}\left(V_{\ell}\right),-t\right)}=1+m t+\frac{m(m+1)}{2} t^{2}+\left(m^{2}+\delta_{m-1} \cdot \frac{m^{2}-1}{8}\right) t^{3}+c t^{4}+O\left(t^{5}\right),
$$

where $m=\ell+1$ and $c=m-\frac{m\left(m^{2}-1\right)\left(m-4-\delta_{m-1}\right)}{4}$. This implies that $c<0$ for all $\ell \geq 4$. In the remaining case $\ell=3$ it is easy to see that:

$$
\frac{1}{h\left(\Lambda_{\sigma}\left(V_{3}\right),-t\right)}=1+4 t+10 t^{2}+16 t^{3}+4 t^{4}-80 t^{5}+O\left(t^{6}\right) .
$$

Therefore, (2.20) makes no sense for $\ell \geq 3$, and $S_{\sigma}\left(V_{\ell}\right)$ is not Koszul. The corollary is proved.

We will conclude the section with a yet conjectural computation of the Hilbert series of (non-Koszul) quadratic algebras $S_{\sigma}\left(V_{\ell}\right)$.

Corollary 2.39 (from Conjecture 2.37). (a) If $\ell$ is odd, then

$$
h\left(S_{\sigma}\left(V_{\ell}\right), t\right)=1+(\ell+1) \frac{t}{1-t}+\left(\begin{array}{c}
\ell+1 \\
2
\end{array}\right) \frac{t^{2}}{(1-t)^{2}} .
$$

(b) If $\ell$ is even, then

$$
h\left(S_{\sigma}\left(V_{\ell}\right), t\right)=1+\ell t+\frac{t}{1-t}+\left(\left(\begin{array}{c}
\frac{\ell}{2} \\
2
\end{array}\right)+\ell\right)\left(\frac{t^{2}}{1-t}+\frac{t^{2}}{(1-t)^{2}}\right)+\frac{\ell^{2}}{4} \frac{t^{2}}{(1-t)^{3}} .
$$

Proof. Prove (a) first. According to (2.19), one has for $n \geq 1$ :

$$
\operatorname{dim}\left(S_{\sigma} V_{\ell}\right)_{n}=\operatorname{dim} S_{\sigma}^{n} V_{\ell}=\frac{(\ell+1)(\ell(n-1)+2)}{2}=\left(\begin{array}{c}
\ell+1 \\
2
\end{array}\right)(n-1)+\ell+1 .
$$

Therefore the Hilbert series of the co-algebra $S_{\sigma} V_{\ell}$ is given by:

$$
h\left(S_{\sigma}\left(V_{\ell}\right), t\right)=1+\sum_{n \geq 1}\left(\begin{array}{c}
\ell+1 \\
2
\end{array}\right)(n-1) t^{n}+(\ell+1) t^{n}=1+(\ell+1) \frac{t}{1-t}+\left(\begin{array}{c}
\ell+1 \\
2
\end{array}\right) \frac{t^{2}}{(1-t)^{2}} .
$$

Part (a) is proved. Prove (b) now. Using (2.19) again, one has for $n \geq 2$ :

$$
\operatorname{dim} S_{\sigma}^{n} V_{\ell}=\left(\begin{array}{c}
\frac{n \ell}{2}+2 \\
2
\end{array}\right)=\frac{\ell^{2}}{4}\left(\begin{array}{l}
n \\
2
\end{array}\right)+\frac{\ell^{2}+6 \ell}{8} n+1 .
$$

Therefore,

$$
\begin{gathered}
h\left(S_{\sigma}\left(V_{\ell}\right), t\right)=1+\left((\ell+1) t-\left(\begin{array}{c}
\frac{\ell}{2}+2 \\
2
\end{array}\right)\right)+\sum_{n \geq 1} \frac{\ell^{2}}{4}\left(\begin{array}{l}
n \\
2
\end{array}\right) t^{n}+\frac{\ell^{2}+6 \ell}{8} n t^{n}+t^{n} \\
=1+\ell t+\frac{t}{1-t}+\left(\left(\begin{array}{c}
\frac{\ell}{2} \\
2
\end{array}\right)+\ell\right)\left(\frac{t^{2}}{1-t}+\frac{t^{2}}{(1-t)^{2}}\right)+\frac{\ell^{2}}{4} \frac{t^{2}}{(1-t)^{3}} .
\end{gathered}
$$

This proves part (b). The corollary is proved.

This result implies, in particular, that the associated scheme $X\left(\bar{V}_{\ell}\right)$ (see Problem 2.23) is a curve if $\ell$ is odd and a surface if $\ell$ is even.

Remark 2.40. It follows from Corollary 2.39 that the Hilbert series for $S_{\sigma}\left(V_{2 k}\right)$ is regular at $\infty$ and $\lim _{t \rightarrow \infty} h\left(S_{\sigma}\left(V_{2 k}\right), t\right)=\left(\begin{array}{c}2 k \\ 2\end{array}\right)$. We expect that this phenomenon is caused by the vanishing of the cubic component in the dual algebra $\Lambda_{\sigma}\left(V_{2 k}^{*}\right)=$ $S_{\sigma}\left(V_{2 k}\right)^{!}$(Theorem 2.35). 


\section{Proofs of MAIN RESUlts}

3.1. Bracketed superalgebras and the proof of Theorem 2.18, We first formulate and prove a number of results about bracketed superalgebras. The following fact is obvious:

Lemma 3.1. The tensor product of bracketed superalgebras is a bracketed superalgebra, and the tensor product of Poisson superalgebras is a Poisson superalgebra.

Definition 3.2. We say that a bracketed superalgebra $A=A_{\overline{0}} \oplus A_{\overline{1}}$ is homogeneous if:

(i) $A_{\overline{0}}=\bigoplus_{n \geq 0} A_{\overline{0}, n}, A_{1}=\bigoplus_{n \geq 0} A_{\overline{1}, n}$ is $\mathbb{Z}_{2} \times \mathbb{Z}_{\geq 0}$-grading of $A$ as algebra, i.e., $A_{\varepsilon, m} \cdot A_{\delta, n} \subset A_{\varepsilon+\delta, m+n}$ for any $\varepsilon, \delta \in \mathbb{Z}_{2}=\mathbb{Z} /(2)=\{\overline{0}, \overline{1}\}$ and any $m, n \in \mathbb{Z}_{\geq 0}$;

(ii) $A$ is generated by $A_{\overline{0}, 1} \oplus A_{\overline{1}, 1}$ as an algebra;

(iii) $\left\{A_{\varepsilon, m}, A_{\delta, n}\right\} \subset A_{\varepsilon+\delta, m+n}$ for any $\varepsilon, \delta \in \mathbb{Z}_{2}$ and any $m, n \in \mathbb{Z}_{\geq 0}$.

The following fact is obvious.

Lemma 3.3. The tensor product of homogeneous bracketed superalgebras is also a homogeneous bracketed superalgebra.

For any homogeneous bracketed superalgebra $A$ define the subspaces $I_{n} \subset A_{n}$, $n=3,4, \ldots$, recursively:

$$
I_{3}(A)=\operatorname{Span}\left\{J(a, b, c) \mid a, b, c \in A_{\overline{0}, 1}+A_{\overline{1}, 1}\right\}
$$

and $I_{n+1}(A)=\left\{A_{\overline{0}, 1}+A_{\overline{1}, 1}, I_{n}(A)\right\}$ for $n \geq 3$. Denote by $I(A)$ the (super)ideal generated by all $I_{n}(A)$. We say that a (super)ideal $J \subset A$ is Poisson if it contains $I_{3}(A)$ and $\{A, J\} \subset J$. Equivalently, $J$ is Poisson if the quotient superalgebra $A / J$ is naturally bracketed and Poisson.

Proposition 3.4. For any homogeneous bracketed superalgebra one has

(a) $I(A)$ is Poisson.

(b) Any Poisson (super)ideal $J$ in A contains $I(A)$.

Proof. We need the following fact.

Lemma 3.5. Let $A$ be a homogeneous bracketed superalgebra. Then $A$ is Poisson if and only if $J(u, v, w)=0$ for all $u, v, w \in A_{\overline{0}, 1}+A_{\overline{1}, 1}$.

Proof. One easily checks that for any $a \in A_{\varepsilon}, b \in A_{\delta}, c \in A_{\gamma}$, and $d \in A_{\theta}$ one has

$$
J(a, b, c \cdot d)=(-1)^{\delta \gamma} c J(a, b, d)+(-1)^{\varepsilon \theta} J(a, b, c) d
$$

and $J(c, a, b)=J(a, b, c)$. An obvious induction by the degree (defined via the direct sum decomposition of $A$ ) completes the proof.

This proves part (a). Part (b) is obvious because by the very definition, $I(A)$ is the smallest Poisson ideal in $A$.

Definition 3.6. We say that a homogeneous bracketed superalgebra is almost Poisson, if the ideal $I(A)$ is generated by its third component $I_{3}(A)$, i.e., the obstruction for the algebra $A$ to be Poisson lies entirely in the 3rd degree.

Lemma 3.7. The tensor product of two almost Poisson superalgebras is almost Poisson. 
Proof. It is easy to see that $I_{3}(A \otimes B)=I_{3}(A) \otimes 1+1 \otimes I_{3}(B)$ for any homogeneous bracketed superalgebras $A$ and $B$. That is,

$$
I_{3}(A \otimes B) \cdot(A \otimes B)=I_{3}(A) \cdot A \otimes B+A \otimes I_{3}(B) \cdot B=I(A) \otimes B+A \otimes I(B)
$$

because $I(A)=I_{3}(A) \cdot A$ and $I(A)=I_{3}(A) \cdot A$. Therefore, by Proposition 3.9.

$$
I_{3}(A \otimes B) \cdot(A \otimes B)=I(A \otimes B) .
$$

The lemma is proved.

For a homogeneous bracketed superalgebra $A$ denote $P(A):=A / I(A)$ and refer to $P(A)$ as the Poisson closure of $A$.

The following result is an immediate corollary of Proposition 3.4 .

Corollary 3.8. The Poisson closure $P(A)$ is characterized by the following universal property: Let $P$ be any Poisson superalgebra. Then any homomorphism of bracketed superalgebras $f: A \rightarrow P$ factors through $P(A)$, i.e., there exists a surjective homomorphism $\pi: A \rightarrow P(A)$ of bracketed algebras and a homomorphism $j: P(A) \rightarrow P$ of Poisson algebras such that $f=j \circ \pi$.

Clearly, the correspondence $A \mapsto P(A)$ is a functor from the category of homogeneous bracketed superalgebras to the category of Poisson superalgebras.

Proposition 3.9. Let $A$ and $B$ be homogeneous bracketed superalgebras. Then one has a canonical isomorphism of Poisson algebras: $P(A \otimes B)=P(A) \otimes P(B)$. Equivalently, $I(A \otimes B)=I(A) \otimes B+A \otimes I(B)$.

Proof. By Lemma 3.1, $P(A) \otimes P(B)$ is Poisson as a product of Poisson superalgebras. One also has a surjective algebra homomorphism $A \otimes B \rightarrow P(A) \otimes P(B)$. Due to the universality of the Poisson closure $P(A \otimes B)$, the latter homomorphism factors through the homomorphism $P(A \otimes B) \rightarrow P(A) \otimes P(B)$ of Poisson algebras. Therefore,

$$
I(A \otimes B) \subseteq I(A) \otimes B+A \otimes I(B) .
$$

On the other hand, $I_{3}(A \otimes B)$ contains both $I_{3}(A \otimes 1)=I_{3}(A) \otimes 1$ and $I_{3}(1 \otimes B)=$ $1 \otimes I_{3}(B)$. This implies that $I_{n}(A \otimes B)$ contains both $I_{n}(A) \otimes 1$ and $1 \otimes I_{n}(B)$. Therefore, $I(A \otimes B)$ contains both $I(A) \otimes 1$ and $1 \otimes I(B)$, and we obtain the opposite inclusion:

$$
I(A \otimes B) \supseteq I(A) \otimes B+A \otimes I(B) .
$$

The obtained double inclusion proves the assertion.

Now we are ready to finish the proof of Theorem 2.18.

Let $\mathbf{U}$ be a coalgebra with the co-product $\Delta: \mathbf{U} \rightarrow \mathbf{U} \otimes \mathbf{U}$ and let $A=\bigoplus_{n \geq 0} A_{n}$

(where $A_{n}=A_{\overline{0}, n} \oplus A_{\overline{1}, n}$ ) be a homogeneous bracketed superalgebra. We say that $\mathbf{U}$ is acting on $A$ if $A$ is a $\mathbf{U}$-module algebra, $\mathbf{U}\left(A_{\varepsilon, n}\right) \subset A_{\varepsilon, n}$ for each $\varepsilon \in \mathbb{Z}_{2}$, $n \geq 0$, and the bracket satisfies:

$$
\{u, v\}=\mathbf{m}_{2}(\mathbf{r}(u \otimes v))
$$

for each $u, v \in A_{1}$, where $\mathbf{m}_{2}: A_{1} \otimes A_{1} \rightarrow A_{2}$ is the multiplication, and $\mathbf{r} \in \mathbf{U} \wedge \mathbf{U}$ is an element such that $(\Delta \otimes i d)(\mathbf{r})=\mathbf{r}_{13}+\mathbf{r}_{23}$. 
Proposition 3.10. Let $\mathbf{U}$ be a co-algebra acting on a homogeneous bracketed superalgebra $A$. Then:

(a) $\{a, b\}=\mathbf{m}(\mathbf{r}(a \otimes b))$ for all $a, b \in A$, where $\mathbf{m}: A \otimes A \rightarrow A$ is the multiplication.

(b) $\{B, C\} \subset B \cdot C$ for any $\mathbf{U}$-submodules $B$ and $C$ of $A$. In particular, $A$ is almost Poisson.

Proof. Prove (a). We will proceed by induction on degree. Indeed, let $a \in A_{\varepsilon, n_{1}}$, $b \in A_{\delta, n_{2}}, c \in A_{n_{3}}$ such that $n_{1} \leq n, n_{2}+n_{3}=n, n_{2}>0, n_{3}>0$. The inductive hypothesis (applied when $n_{2}<n$ ) reads:

$$
\{a, b\}=\mathbf{r}_{(1)}(a) \cdot \mathbf{r}_{(2)}(b),\{a, c\}=\mathbf{r}_{(1)}(a) \cdot \mathbf{r}_{(2)}(c),
$$

where we have used the Sweedler notation $\mathbf{r}=\mathbf{r}_{(1)} \otimes \mathbf{r}_{(2)}$. Then

$$
\begin{gathered}
\{a, b c\}=\{a, b\} c+(-1)^{\varepsilon \delta} b\{a, c\}=\mathbf{r}_{(1)}(a) \cdot \mathbf{r}_{(2)}(b) \cdot c+(-1)^{\varepsilon \delta} b \cdot \mathbf{r}_{(1)}(a) \cdot \mathbf{r}_{(2)}(c) \\
=\mathbf{r}_{(1)}(a)\left(\mathbf{r}_{(2)}(b) \cdot c+b \cdot \mathbf{r}_{(2)}(c)\right)=\mathbf{m}(\mathbf{r}(a \otimes b c))
\end{gathered}
$$

This proves part (a). To prove (b) note that $\{B, C\} \subset \mathbf{r}_{(1)}(B) \cdot \mathbf{r}_{(2)}(C) \subset B$. $C$ because $\mathbf{r}_{(1)}(B) \subset B, \mathbf{r}_{(2)}(C) \subset C$. In particular, taking $B=\bar{V}$ and $C=$ $J(\bar{V}, \bar{V}, \bar{V})$ proves that $A$ is almost Poisson. The proposition is proved.

Now we will set $\mathbf{U}:=U(\mathfrak{g}), \mathbf{r}=r^{-}=r-\tau(r)$. Then setting respectively $A=$ $S(\bar{V})$ and $A=\Lambda(\bar{V})$ with the bracket given by (3.1) and using Proposition 3.10(a) finishes the proof of Theorem 2.18(a). Then Proposition 3.10(b) guarantees that both $S(\bar{V})$ and $\Lambda(\bar{V})$ are almost Poisson in the sense of Definition 3.6. Therefore, $\overline{S(\bar{V})}$ and $\overline{S(\bar{V})}$ are Poisson superalgebras. This finishes the proof of Theorem 2.18 (b).

Theorem 2.18 is proved.

3.2. (k, A)-algebras and the proof of Theorem 2.21, In this section we will develop a general framework of $(\mathbf{k}, \mathbf{A})$-algebras and will prove Theorem 2.21 along with its generalizations in the category of $(\mathbf{k}, \mathbf{A})$-algebras.

Let $\mathbf{k}$ be a field and $\mathbf{A}$ be a local subring of $\mathbf{k}$. Denote by $\mathfrak{m}$ the only maximal ideal in $\mathbf{A}$ and by $\tilde{\mathbf{k}}$ the residue field of $\mathbf{A}$, i.e., $\tilde{\mathbf{k}}:=\mathbf{A} / \mathfrak{m}$.

We say that an $\mathbf{A}$-submodule $L$ of a k-vector space $V$ is an $\mathbf{A}$-lattice of $V$ if $L$ is a free $\mathbf{A}$-module and $\mathbf{k} \otimes_{\mathbf{A}} L=V$, i.e., $L$ spans $V$ as a $\mathbf{k}$-vector space. Note that for any k-vector space $V$ and any $\mathbf{k}$-linear basis $\mathbf{B}$ of $V$ the $\mathbf{A}$-span $L=\mathbf{A} \cdot \mathbf{B}$ is an A-lattice in $V$. Conversely, if $L$ is an $\mathbf{A}$-lattice in $V$, then any $\mathbf{A}$-linear basis $\mathbf{B}$ of $L$ is also a $\mathbf{k}$-linear basis of $V$.

Denote by $(\mathbf{k}, \mathbf{A})-\operatorname{Mod}$ the category whose objects are pairs $\mathcal{V}=(V, L)$ where $V$ is a $\mathbf{k}$-vector space and $L \subset V$ is an A-lattice of $V$; an arrow $(V, L) \rightarrow\left(V^{\prime}, L^{\prime}\right)$ is any k-linear map $f: V \rightarrow V^{\prime}$ such that $f(L) \subset L^{\prime}$.

Clearly, $(\mathbf{k}, \mathbf{A})-\operatorname{Mod}$ is an abelian category. Moreover, $(\mathbf{k}, \mathbf{A})-\operatorname{Mod}$ is $\mathbf{A}$-linear because each $\operatorname{Hom}(\mathcal{U}, \mathcal{V})$ in $(\mathbf{k}, \mathbf{A})-\operatorname{Mod}$ is an A-module.

Definition 3.11. We say that a functor $F: \mathcal{C} \rightarrow \mathcal{D}$ is an almost equivalence of $\mathcal{C}$ and $\mathcal{D}$ if:

(a) for any objects $c, c^{\prime}$ of $\mathcal{C}$ an isomorphism $F(c) \cong F\left(c^{\prime}\right)$ in $\mathcal{D}$ implies that $c \cong c^{\prime}$ in $\mathcal{C}$;

(b) for any object in $d$ there exists an object $c$ in $\mathcal{C}$ such that $F(c) \cong d$ in $\mathcal{D}$. 
Lemma 3.12. The forgetful functor $(\mathbf{k}, \mathbf{A})-M o d \rightarrow \mathbf{k}-M o d$ given by $(V, L) \mapsto V$ is an almost equivalence of categories.

Proof. It suffices to show that any two objects of the form $(V, L)$ and $\left(V, L^{\prime}\right)$ are isomorphic in $(\mathbf{k}, \mathbf{A})-M o d$. This follows from the fact that there exist $\mathbf{k}$-linear bases

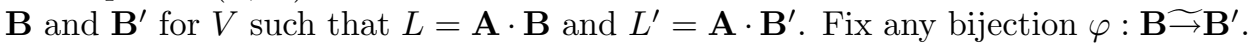
This extends to a k-linear automorphism $f: V \rightarrow V$ such that $f(L)=L^{\prime}$. This $f$ is a desirable isomorphism $(V, L) \widetilde{\rightarrow}\left(V, L^{\prime}\right)$.

The following fact is, apparently, well-known.

Lemma 3.13. (k, A) - Mod is a symmetric tensor category under the operation

$$
(U, L) \otimes\left(V, L^{\prime}\right)=\left(U \otimes_{\mathbf{k}} V, L \otimes_{\mathbf{A}} L^{\prime}\right)
$$

for any objects $(U, L)$ and $\left(V, L^{\prime}\right)$ of $(\mathbf{k}, \mathbf{A})-M o d$.

Proof. Let $(U, L)$ and $\left(V, L^{\prime}\right)$ be objects of $(\mathbf{k}, \mathbf{A})-M o d$. Clearly,

$$
U \otimes_{\mathbf{k}} V=\left(\mathbf{k} \otimes_{\mathbf{A}} L\right) \otimes_{\mathbf{k}}\left(\mathbf{k} \otimes_{\mathbf{A}} L^{\prime}\right) .
$$

This taken together with the following general identity

$$
\left(\mathbf{k} \otimes_{\mathbf{A}} L\right) \otimes_{\mathbf{k}}\left(\mathbf{k} \otimes_{\mathbf{A}} L^{\prime}\right)=\mathbf{k} \otimes_{\mathbf{A}}\left(L \otimes_{\mathbf{A}} L^{\prime}\right)
$$

implies that $L \otimes_{\mathbf{A}} L^{\prime}$ is an A-lattice in $U \otimes_{\mathbf{k}} V$.

The associativity of the product is obvious, and the unit object is $(\mathbf{k}, \mathbf{A})$.

The lemma is proved.

Define a functor $\mathcal{F}:(\mathbf{k}, \mathbf{A})-M o d \rightarrow \tilde{\mathbf{k}}-\operatorname{Mod}$ as follows. For any object $(V, L)$ of $(\mathbf{k}, \mathbf{A})-M o d$ we set $\mathcal{F}(V, L)=L / \mathfrak{m} L$, and for any morphism $f:(V, L) \rightarrow\left(V^{\prime}, L^{\prime}\right)$ we set $\mathcal{F}(f): L / \mathfrak{m} L \rightarrow L^{\prime} / \mathfrak{m} L^{\prime}$ to be a natural $\tilde{\mathbf{k}}$-linear map.

Lemma 3.14. $\mathcal{F}:(\mathbf{k}, \mathbf{A})-\operatorname{Mod} \rightarrow \tilde{\mathbf{k}}-\operatorname{Mod}$ is a tensor functor and an almost equivalence of categories.

Proof. First, let us show that $\mathcal{F}$ is a tensor functor. It follows from the fact that for any A-module $M$ and any A-module $M^{\prime}$ we have a canonical isomorphism (even without any restrictions on a commutative ring $\mathbf{A}$ and an ideal $\mathfrak{m} \subset \mathbf{A}$ ):

$$
(M / \mathfrak{m} M) \bigotimes_{\mathbf{A} / \mathfrak{m}}\left(M^{\prime} / \mathfrak{m} M^{\prime}\right)=\left(M \bigotimes_{\mathbf{A}} M\right) /\left(\mathfrak{m} M \bigotimes_{\mathbf{A}} M^{\prime}\right) .
$$

The fact that $\mathcal{F}$ is an almost equivalence of categories follows from the following two observations:

1. $(V, L) \cong\left(V^{\prime}, L^{\prime}\right)$ in $(\mathbf{k}, \mathbf{A})-M o d$ if and only if $V \cong V^{\prime}$ as $\mathbf{k}$-vector spaces. This is a direct consequence of the proof of Lemma 3.12

2. For any basis $\overline{\mathbf{B}}$ of $\bar{V}$ in $\tilde{\mathbf{k}}-\operatorname{Mod}$ we have that $\left(\operatorname{Span}_{\mathbf{k}}[\overline{\mathbf{B}}], \operatorname{Span}_{\mathbf{A}}[\overline{\mathbf{B}}]\right)$ is an object of $(\mathbf{k}, \mathbf{A})-\operatorname{Mod}$ and $\mathcal{F}\left(\operatorname{Span}_{\mathbf{k}}[\overline{\mathbf{B}}], \operatorname{Span}_{\mathbf{A}}[\overline{\mathbf{B}}]\right)=\operatorname{Span}_{\tilde{\mathbf{k}}}\{\overline{\mathbf{B}}\} \cong \bar{V}$.

The lemma is proved.

In what follows we will sometimes abbreviate $\bar{V}=\mathcal{F}(V, L)$ and $\bar{f}=\mathcal{F}(f)$. Similarly, for an algebra $\mathcal{A}=\left(A, L_{A}\right)$ in the $(\mathbf{k}, \mathbf{A})-M o d$ we will sometimes abbreviate $\overline{\mathcal{A}}=\mathcal{F} \mathcal{A}$, the algebra over the residue field $\tilde{\mathbf{k}}=\mathbf{A} / \mathfrak{m}$.

Let $U$ be a k-Hopf algebra and let $U_{\mathbf{A}}$ be a Hopf A-subalgebra of $U$. This means that $\Delta\left(U_{\mathbf{A}}\right) \subset U_{\mathbf{A}} \otimes_{\mathbf{A}} U_{\mathbf{A}}$ (where $U_{\mathbf{A}} \otimes_{\mathbf{A}} U_{\mathbf{A}}$ is naturally an $\mathbf{A}$-subalgebra of $U \otimes_{\mathbf{k}} U$ ), $\varepsilon\left(U_{\mathbf{A}}\right) \subset \mathbf{A}$, and $S\left(U_{\mathbf{A}}\right) \subset U_{\mathbf{A}}$. We will refer to the above pair $\mathcal{U}=\left(U, U_{\mathbf{A}}\right)$ as 
$(\mathbf{k}, \mathbf{A})$-Hopf algebra (please note that $U_{\mathbf{A}}$ is not necessarily a free $\mathbf{A}$-module, that is, $\mathcal{U}$ is not necessarily a $(\mathbf{k}, \mathbf{A})$-module).

Given a $(\mathbf{k}, \mathbf{A})$-Hopf algebra $\mathcal{U}=\left(U, U_{\mathbf{A}}\right)$, we say that an object $\mathcal{V}=(V, L)$ of $(\mathbf{k}, \mathbf{A})-M o d$ is a $\mathcal{U}$-module if $V$ is a $U$-module and $L$ is a $U_{\mathbf{A}}$-module.

Denote by $\mathcal{U}-\operatorname{Mod}$ the category to which objects are $\mathcal{U}$-modules and arrows are those morphisms of $(\mathbf{k}, \mathbf{A})$-modules which commute with the $\mathcal{U}$-action.

Clearly, for $(\mathbf{k}, \mathbf{A})$-Hopf algebra $\mathcal{U}=\left(U, U_{\mathbf{A}}\right)$ the category $\mathcal{U}-M o d$ is a tensor (but not necessarily symmetric) category.

For each $(\mathbf{k}, \mathbf{A})$-Hopf algebra $\mathcal{U}=\left(U, U_{\mathbf{A}}\right)$ we define $\overline{\mathcal{U}}:=U_{\mathbf{A}} / \mathfrak{m} U_{\mathbf{A}}$. Clearly, $\overline{\mathcal{U}}$ is a Hopf algebra over $\tilde{\mathbf{k}}=\mathbf{A} / \mathfrak{m}$.

The following fact is obvious.

Lemma 3.15. In the notation of Lemma 3.14, for any $(\mathbf{k}, \mathbf{A})$-Hopf algebra $\mathcal{U}$ the functor $\mathcal{F}$ naturally extends to a tensor functor

$$
\mathcal{U}-\operatorname{Mod} \rightarrow \overline{\mathcal{U}}-\operatorname{Mod} .
$$

Now let $\mathbf{k}=\mathbb{C}(q)$, and $\mathbf{A}$ is the ring of all those rational functions in $q$ which are defined at $q=1$. Clearly, $\mathbf{A}$ is a local PID with maximal ideal $\mathfrak{m}=(q-1) \mathbf{A}$ (and, moreover, each ideal in $\mathbf{A}$ is of the form $\left.\mathfrak{m}^{n}=(q-1)^{n} \mathbf{A}\right)$. Therefore, $\tilde{\mathbf{k}}:=\mathbf{A} / \mathfrak{m}=\mathbb{C}$.

Recall from Section 1 that $U_{q}(\mathfrak{g})$ is the quantized universal enveloping algebra (of a reductive Lie algebra Let $\mathfrak{g}$ ) generated (over $\mathbf{k}=\mathbb{C}(q)$ ) by $E_{i}, F_{i}, i=1,2, \ldots, r$, and all $K_{\lambda}, \lambda \in P$. Denote $h_{\lambda}=\frac{K_{\lambda}-1}{q-1}$ and let $U_{\mathbf{A}}(\mathfrak{g})$ be the A-algebra generated by all $h_{\lambda}, \lambda \in P$ and all $E_{i}, F_{i}$.

Denote by $\mathcal{U}_{q}(\mathfrak{g})$ the pair $\left(U_{q}(\mathfrak{g}), U_{\mathbf{A}}(\mathfrak{g})\right)$.

Lemma 3.16. The pair $\mathcal{U}_{q}(\mathfrak{g})=\left(U_{q}(\mathfrak{g}), U_{\mathbf{A}}(\mathfrak{g})\right)$ is a $(\mathbf{k}, \mathbf{A})$-Hopf algebra.

Proof. All we have to show is that $U_{\mathbf{A}}(\mathfrak{g})$ is a Hopf A-subalgebra of $U_{q}(\mathfrak{g})$. That is, we have to show that $U_{\mathbf{A}}(\mathfrak{g})$ is closed under the coproduct, the counit and the antipode. Clearly, $\Delta\left(E_{i}\right), \Delta\left(F_{i}\right) \in U_{\mathbf{A}}(\mathfrak{g}) \otimes_{\mathbf{A}} U_{\mathbf{A}}(\mathfrak{g}), \varepsilon\left(E_{i}\right), \varepsilon\left(F_{i}\right) \in \mathbf{A}$, and $S\left(E_{i}\right), S\left(F_{i}\right) \in U_{\mathbf{A}}(\mathfrak{g})$ for all $\lambda \in P$ and $i=1,2, \ldots, r$. Therefore, it remains to show that $\Delta\left(h_{\lambda}\right) \in U_{\mathbf{A}}(\mathfrak{g}) \otimes_{\mathbf{A}} U_{\mathbf{A}}(\mathfrak{g}), \epsilon\left(h_{\lambda}\right) \in \mathbf{A}$, and $S\left(h_{\lambda}\right) \in U_{\mathbf{A}}(\mathfrak{g})$. It is easy to see that

$$
\begin{aligned}
& \Delta\left(h_{\lambda}\right)=\frac{K_{\lambda} \otimes K_{\lambda}-1 \otimes 1}{q-1}=h_{\lambda} \otimes K_{\lambda}+1 \otimes h_{\lambda}, \\
& \epsilon\left(h_{\lambda}\right)=0, S\left(h_{\lambda}\right)=-h_{\lambda} K_{-\lambda} .
\end{aligned}
$$

This proves the lemma because $K_{\lambda}=(q-1) h_{\lambda}+1$, e.g., all $K_{\lambda} \in U_{\mathbf{A}}(\mathfrak{g})$.

Lemma 3.17. We have $\overline{\mathcal{U}_{q}(\mathfrak{g})}=U(\mathfrak{g})$.

Proof. The Hopf algebra $\overline{\mathcal{U}_{q}(\mathfrak{g})}$ is generated (over $\tilde{\mathbf{k}}=\mathbb{C}$ ) by

$$
\bar{h}_{\lambda}=h_{\lambda} \bmod (q-1) U_{\mathbf{A}}(\mathfrak{g}), \bar{K}_{\lambda}=K_{\lambda} \bmod (q-1) U_{\mathbf{A}}(\mathfrak{g})
$$

for $\lambda \in P$ and by

$$
\bar{E}_{i}=E_{i} \quad \bmod (q-1) U_{\mathbf{A}}(\mathfrak{g}), \bar{F}_{i}=F_{i} \quad \bmod (q-1) U_{\mathbf{A}}(\mathfrak{g})
$$

for $i=1, \ldots, r$. Note that $\bar{K}_{\lambda}=1$ for all $\lambda$ because $K_{\lambda}=(q-1) h_{\lambda}+1$. Furthermore, the relations

$$
h_{\lambda+\mu}=(q-1) h_{\lambda} h_{\mu}+h_{\lambda}+h_{\mu}
$$


imply that $\bar{h}_{\lambda+\mu}=\bar{h}_{\lambda}+\bar{h}_{\mu}$ for all $\lambda, \mu \in P$. That is, the $\mathbb{C}$-span of all $\bar{h}_{\lambda}$ is the Cartan subalgebra of $\mathfrak{g}$. Next, the relations in $\mathcal{U}_{q}(\mathfrak{g})$ :

$$
h_{\lambda} E_{i}-E_{i} h_{\lambda}=\frac{q^{\left(\lambda \mid \alpha_{i}\right)}-1}{q-1} E_{i} K_{\lambda}, h_{\lambda} F_{i}-F_{i} h_{\lambda}=\frac{q^{-\left(\lambda \mid \alpha_{i}\right)}-1}{q-1} F_{i} K_{\lambda}
$$

yield

$$
\bar{h}_{\lambda} \bar{E}_{i}-\bar{E}_{i} \bar{h}_{\lambda}=\left(\lambda \mid \alpha_{i}\right) \bar{E}_{i}, \bar{h}_{\lambda} \bar{F}_{i}-\bar{F}_{i} \bar{h}_{\lambda}=-\left(\lambda \mid \alpha_{i}\right) \bar{F}_{i} .
$$

Furthermore, the relation (1.1) in $\mathcal{U}_{\mathbf{A}}(\mathfrak{g})$ :

$$
E_{i} F_{j}-F_{j} E_{i}=\delta_{i, j} \frac{K_{\alpha_{i}}-K_{-\alpha_{i}}}{q^{d_{i}}-q^{-d_{i}}}=\delta_{i, j} h_{\alpha_{i}} \frac{1+K_{-\alpha_{i}}}{1+q^{-d_{i}}} \cdot \frac{q-1}{q^{d_{i}}-1}
$$

becomes $\bar{E}_{i} \bar{F}_{j}-\bar{F}_{j} \bar{E}_{i}=\delta_{i, j} \frac{1}{d_{i}} \bar{h}_{\alpha_{i}}$.

Also the quantum Serre relations (1.2) in $\mathcal{U}_{\mathbf{A}}(\mathfrak{g})$ turn into the classical Serre relations in $\mathfrak{g}$. Therefore, if we denote $\bar{H}_{i}=\frac{1}{d_{i}} \bar{h}_{\alpha_{i}}$, then the elements $\bar{E}_{i}, \bar{F}_{i}, \bar{H}_{i}$ become the standard Chevalley generators of the semisimple part of $\mathfrak{g}$.

This proves that the Hopf algebra $\overline{\mathcal{U}_{q}(\mathfrak{g})}$ is naturally isomorphic to the universal enveloping algebra $U(\mathfrak{g})$. The lemma is proved.

Therefore, based on Lemmas 3.15 3.16, and 3.17 we define the "dequantization" functor $\mathcal{U}_{q}(\mathfrak{g})-\operatorname{Mod} \rightarrow U(\mathfrak{g})-\operatorname{Mod}$.

Denote by $\mathcal{O}_{f}\left(\mathcal{U}_{q}(\mathfrak{g})\right)$ the full sub-category of $\mathcal{U}_{q}(\mathfrak{g})-\operatorname{Mod}$ whose objects $(V, L)$ are such that $V$ is an object of $\mathcal{O}_{f}$ and $L$ is an A-lattice in $V$ compatible with the weight decomposition $V=\bigoplus_{\mu \in P} V(\mu)$, i.e., each $L(\mu):=V(\mu) \cap L$ is an A-lattice in $V(\mu)$.

Let $V_{\lambda} \in O b\left(\mathcal{O}_{f}\right)$ be an irreducible $U_{q}(\mathfrak{g})$-module with highest weight $\lambda \in P^{+}$ and let $v_{\lambda} \in V_{\lambda}$ be a highest weight vector. Define $L_{v_{\lambda}}=U_{\mathbf{A}}(\mathfrak{g}) \cdot v_{\lambda}$.

Lemma 3.18. $\left(V_{\lambda}, L_{v_{\lambda}}\right) \in \mathcal{O}_{f}\left(\mathcal{U}_{q}(\mathfrak{g})\right)$.

Proof. Clearly, $\mathbf{k} \otimes_{\mathbf{A}} L_{v_{\lambda}}=V_{\lambda}$. It remains to show that $L_{v_{\lambda}}$ is a free A-module. It is easy to see that $U_{\mathbf{A}}(\mathfrak{g})\left(v_{\lambda}\right)=U_{\mathbf{A}}^{-}\left(v_{\lambda}\right)$, where $U_{\mathbf{A}}^{-}$is the A-subalgebra of $U_{\mathbf{A}}(\mathfrak{g})$ generated by $F_{1}, F_{2}, \ldots, F_{r}$. Clearly, $U_{\mathbf{A}}^{-}$is graded via $\operatorname{deg}\left(F_{i}\right)=1: U_{\mathbf{A}}^{-}=$ $\bigoplus_{n \geq 0}\left(U_{\mathbf{A}}^{-}\right)_{n}$, where each $\left(U_{\mathbf{A}}^{-}\right)_{n}$ is a finitely generated A-module. Clearly, $\left(U_{\mathbf{A}}^{-}\right)_{n}\left(v_{\lambda}\right)=0$ for $n>>0$. Therefore, $L_{v_{\lambda}}$ is a finitely generated A-module of finite rank. Finally, since $\mathbf{A}$ is a PID, this implies that $L_{v_{\lambda}}$ is a free $\mathbf{A}$-module. The lemma is proved.

The following fact is obvious and, apparently, well-known.

Lemma 3.19. (a) Each object $\left(V_{\lambda}, L_{v_{\lambda}}\right)$ is irreducible in $\mathcal{O}_{f}\left(\mathcal{U}_{q}(\mathfrak{g})\right)$, and each irreducible object of $\mathcal{O}_{f}\left(\mathcal{U}_{q}(\mathfrak{g})\right)$ is isomorphic to one of $\left(V_{\lambda}, L_{v_{\lambda}}\right)$.

(b) The category $\mathcal{O}_{f}\left(\mathcal{U}_{q}(\mathfrak{g})\right)$ is semisimple.

(c) The forgetful functor $(V, L) \mapsto V$ is an almost equivalence of tensor categories $\mathcal{O}_{f}\left(\mathcal{U}_{q}(\mathfrak{g})\right) \rightarrow \mathcal{O}_{f}$

The following fact shows that the category $\mathcal{O}_{f}\left(\mathcal{U}_{q}(\mathfrak{g})\right)$ is naturally braided.

Lemma 3.20. Let $\mathcal{V}=(V, L)$ and $\mathcal{V}^{\prime}=\left(V^{\prime}, L^{\prime}\right)$ be objects of $\mathcal{O}_{f}\left(\mathcal{U}_{q}(\mathfrak{g})\right)$. Then:

(a) The braiding operator $\mathcal{R}_{V, V^{\prime}}: V \otimes_{\mathbf{k}} V^{\prime} \rightarrow V^{\prime} \otimes_{\mathbf{k}} V$ satisfies:

$$
\mathcal{R}_{V, V^{\prime}}\left(L \otimes_{\mathbf{A}} L^{\prime}\right)=L^{\prime} \otimes_{\mathbf{A}} L .
$$


(b) The operator $\mathcal{R}_{V, V^{\prime}}$ extends to an operator:

$$
\mathcal{R}_{\mathcal{V}, \mathcal{V}^{\prime}}: \mathcal{V} \otimes \mathcal{V}^{\prime} \rightarrow \mathcal{V}^{\prime} \otimes \mathcal{V}
$$

and the collection of all $\mathcal{R}_{\mathcal{V}, \mathcal{V}^{\prime}}$ constitutes a braiding on $\mathcal{O}_{f}\left(\mathcal{U}_{q}(\mathfrak{g})\right)$.

(c) The forgetful functor $(V, L) \mapsto V$ is an almost equivalence of braided tensor categories $\mathcal{O}_{f}\left(\mathcal{U}_{q}(\mathfrak{g})\right) \rightarrow \mathcal{O}_{f}$.

Proof. Note that the universal $R$-matrix $R \in U_{q}(\mathfrak{g}) \widehat{\otimes}_{\mathbf{k}} U_{q}(\mathfrak{g})$ belongs, in fact, to $U_{\mathbf{A}}(\mathfrak{g}) \widehat{\otimes}_{\mathbf{A}} U_{\mathbf{A}}(\mathfrak{g})$. Recall also that $R$ acts on $V \otimes_{\mathbf{k}} V^{\prime}$. Therefore, $R\left(L \otimes_{\mathbf{A}} L^{\prime}\right)=$ $L \otimes_{\mathbf{A}} L^{\prime}$ and $\mathcal{R}_{V, V^{\prime}}\left(L \otimes_{\mathbf{A}} L^{\prime}\right)=\tau R\left(L \otimes_{\mathbf{A}} L^{\prime}\right)=\tau\left(L \otimes_{\mathbf{A}} L^{\prime}\right)=L^{\prime} \otimes_{\mathbf{A}} L$. This proves (a).

Parts (b) and (c) also follow.

Denote by $\overline{\mathcal{O}}_{f}$ the full sub-category of $U(\mathfrak{g})-\operatorname{Mod}$, whose objects $\bar{V}$ are finitedimensional $U(\mathfrak{g})$-modules having a weight decomposition $V=\bigoplus_{\mu \in P} V(\mu)$.

Lemma 3.21. (a) The restriction of the functor $\mathcal{U}_{q}(\mathfrak{g})-\operatorname{Mod} \rightarrow U(\mathfrak{g})-\operatorname{Mod}$ defined by (3.3) to the sub-category $\mathcal{O}_{f}\left(\mathcal{U}_{q}(\mathfrak{g})\right)$ is a tensor functor

$$
\mathcal{O}_{f}\left(\mathcal{U}_{q}(\mathfrak{g})\right) \rightarrow \overline{\mathcal{O}}_{f}
$$

(b) The functor (3.4) is an almost equivalence of categories.

Proof. Prove (a). It suffices to show that for each object $(V, L)$ in $\mathcal{O}_{f}\left(\mathcal{U}_{q}(\mathfrak{g})\right)$ the corresponding $U(\mathfrak{g})$-module $\bar{V}=L / \mathfrak{m} L$ has a weight decomposition. By definition of $\mathcal{O}_{f}\left(\mathcal{U}_{q}(\mathfrak{g})\right)$, the A-lattice $L$ has a weight decomposition $L=\bigoplus_{\mu \in P} L(\mu)$, and for any $\mu, \nu \in P$ and any weight vector $v \in L(\mu)$ one has

$$
h_{\nu} v=\frac{q^{(\mu, \nu)}-1}{q-1} v_{\lambda} .
$$

This implies that passing to the quotient by $(q-1) L$, we obtain

$$
\bar{h}_{\nu}(\bar{v})=(\mu, \nu) \bar{v} .
$$

That is, the $U(\mathfrak{g})$-module $\bar{V}$ has a weight decomposition $\bar{V}=\bigoplus_{\mu \in P} \bar{V}(\mu)$. This proves (a). Prove (b) now. Since both categories $\mathcal{O}_{f}\left(\mathcal{U}_{q}(\mathfrak{g})\right)$ and $\overline{\mathcal{O}}_{f}$ are semisimple, it suffices to compute the functor (3.4) on the irreducible objects. In fact, it suffices to prove that $\bar{V}_{\lambda}:=L_{v_{\lambda}} /(q-1) L_{v_{\lambda}}$ is isomorphic to the corresponding irreducible $U(\mathfrak{g})$-module with the highest weight $\lambda$.

Furthermore, if we denote $\bar{v}_{\lambda}=v_{\lambda}+(q-1) L_{v_{\lambda}}$, and for any $u \in U_{\mathbf{A}}(\mathfrak{g})$ we denote $\bar{u}=u+(q-1) U_{\mathbf{A}}(\mathfrak{g})$, then we obtain

$$
\bar{u}\left(\bar{v}_{\lambda}\right)=\left(u+(q-1) U_{\mathbf{A}}(\mathfrak{g})\right)\left(v_{\lambda}+(q-1) L_{v_{\lambda}}\right)=u\left(v_{\lambda}\right)+(q-1) L_{v_{\lambda}} .
$$

This proves that $\bar{V}_{\lambda}$ is a cyclic finite-dimensional $U(\mathfrak{g})$-module with the highest weight $\lambda$. Therefore, $\bar{V}_{\lambda}$ is irreducible. The lemma is proved.

Combining Lemmas 3.19 and 3.21, we obtain an obvious (and well-known) corollary.

Corollary 3.22. The categories $\mathcal{O}_{f}$ and $\overline{\mathcal{O}}_{f}$ are almost equivalent. 
For any object $V$ of $\mathcal{O}_{f}$ written as $V=\bigoplus_{i=1}^{n} V_{\lambda_{i}}$ we will refer to the object $\bar{V}=\bigoplus_{i=1}^{n} \bar{V}_{\lambda_{i}}$ in $\overline{\mathcal{O}}_{f}$ as the classical limit of $V$. Denote by $\mathcal{O}_{g r, f}\left(\mathcal{U}_{q}(\mathfrak{g})\right)$ the sub-category of $\mathcal{U}_{q}(\mathfrak{g})-M o d$ whose objects are $\mathbb{Z}_{\geq 0}$-graded:

$$
V=\bigoplus_{n \in \mathbb{Z}_{\geq 0}} V_{n}
$$

where each $V_{n}$ is an object of $\mathcal{O}_{f}$; and morphisms are those homomorphisms of $\mathcal{U}_{q}(\mathfrak{g})$-modules which preserve the $\mathbb{Z}_{\geq 0}$-grading. Similarly, denote by $\overline{\mathcal{O}}_{g r, f}$ the sub-category of $U(\mathfrak{g})-M o d$ whose objects are $\mathbb{Z}_{\geq 0}$-graded:

$$
\bar{V}=\bigoplus_{n \in \mathbb{Z}_{\geq 0}} \bar{V}_{n}
$$

where each $\bar{V}_{n}$ is an object of $\mathcal{O}_{f}$; and morphisms are those homomorphisms of $U(\mathfrak{g})$-modules which preserve the $\mathbb{Z}_{\geq 0}$-grading.

Lemma 3.23. The functor (3.4) extends to an almost equivalence of tensor categories $\mathcal{O}_{g r, f}\left(\mathcal{U}_{q}(\mathfrak{g})\right) \rightarrow \overline{\mathcal{O}}_{g r, f}$ via

$$
V=\bigoplus_{n \in \mathbb{Z}_{\geq 0}} V_{n} \mapsto \bar{V}=\bigoplus_{n \in \mathbb{Z}_{\geq 0}} \bar{V}_{n}
$$

We will sometimes call $\bar{V}=\bigoplus_{n \in \mathbb{Z} \geq 0} \bar{V}_{n}$ the classical limit of $V=\bigoplus_{n \in \mathbb{Z} \geq 0} V_{n}$.

Let $A=A_{\overline{0}} \oplus A_{\overline{1}}$ be a $\mathbb{Z}_{2}$-graded $\mathbf{k}$-algebra. Define the super-commutator $[\cdot, \cdot \cdot]$ on $A$ by

$$
[a, b]=a b-(-1)^{\varepsilon \delta} b a
$$

for any $a \in A_{\varepsilon}, b \in A_{\delta}$. It is well-known that the super-commutator satisfies the super-Leibniz rule and super-Jacobi identity:

$$
\begin{gathered}
{[a, b c]=[a, b] c+(-1)^{\varepsilon \cdot \delta} b[a, c],} \\
(-1)^{\varepsilon \gamma}[a,[b, c]]+(-1)^{\gamma \delta}[c,[a, b]]+(-1)^{\delta \varepsilon}[b,[c, a]]=0
\end{gathered}
$$

for any $a \in A_{\varepsilon}, b \in A_{\delta}, c \in A_{\gamma}$.

Now let $L$ be an A-lattice in $A$ such that $L$ is closed under multiplication and compatible with the $\mathbb{Z}_{2}$-grading (i.e., the pair $\mathcal{A}=(A, L)$ is a $\mathbb{Z}_{2}$-graded (k, $\mathbf{A}$ )algebra).

Definition 3.24. We say that a $\mathbb{Z}_{2}$-graded $(\mathbf{k}, \mathbf{A})$-algebra $\mathcal{A}=(A, L)$ is an almost commutative superalgebra if $[L, L] \subset \mathfrak{m} L$.

Let $\mathcal{V}=\mathcal{V}_{0} \oplus \mathcal{V}_{1}$ be a $\mathbb{Z}_{2}$-graded $(\mathbf{k}, \mathbf{A})$-module. Let $\mathcal{A}$ be a $\mathbb{Z}_{2}$-graded $(\mathbf{k}, \mathbf{A})$ algebra generated by $\mathcal{V}$ (i.e., there is a surjective homomorphism $T(\mathcal{V}) \rightarrow \mathcal{A}$ of $(\mathbf{k}, \mathbf{A})$-algebras, where $T(\mathcal{V})$ is the tensor algebra of $\mathcal{V})$. For any map $f: L \times L \rightarrow$ $L \cdot L$ we define the $\mathbf{A}$-submodule $I_{f}$ of $L \cdot L$ by

$$
I_{f}=\operatorname{Span}_{\mathbf{A}}\left\{\left[l, l^{\prime}\right]+\mathfrak{m} f\left(l, l^{\prime}\right) \mid l, l^{\prime} \in L\right\}
$$

and then denote $\mathcal{I}_{f}=\left(\mathbf{k} \otimes_{\mathbf{A}} I_{f}, I_{f}\right)$. The following fact is obvious.

Proposition 3.25. Let $\mathcal{A}$ be a $\mathbb{Z}_{2}$-graded $(\mathbf{k}, \mathbf{A})$-algebra generated by a $\mathbb{Z}_{2}$-graded $(\mathbf{k}, \mathbf{A})$-module $\mathcal{V}=(V, L)$ and let $\mathcal{J}$ be any $\mathbb{Z}_{2}$-homogeneous ideal containing $\mathcal{I}_{f}$. Then the quotient superalgebra $\mathcal{A} / \mathcal{J}$ is almost commutative. 
Proof. Let $L^{k}=L \cdot L \cdots L$, where the product is taken $k$ times. We will proceed by induction in $k$. If $k=1$, then

$$
[a, b] \in \mathcal{I}_{f}+\mathfrak{m} L^{2}
$$

for $a, b \in L^{1}=L$, which implies that $[\bar{a}, \bar{b}] \equiv 0 \bmod \mathfrak{m}$ in the quotient algebra $\mathcal{A} / \mathcal{J}$. The inductive step follows directly from the super-Leibniz rule (3.5). This proves the proposition.

Note that the functor $\mathcal{F}$ from Lemma 3.14 takes a $\mathbb{Z}_{2}$-graded $(\mathbf{k}, \mathbf{A})$-superalgebra $\mathcal{A}=\left(A, L_{A}\right)$ into the superalgebra $\mathcal{F}(\mathcal{A})=L_{A} / \mathfrak{m} L_{A}$ over the residue field $\tilde{\mathbf{k}}=\mathbf{k} / \mathfrak{m}$. The following fact is obvious.

Lemma 3.26. For any almost commutative $(\mathbf{k}, \mathbf{A})$-algebra $\mathcal{A}=\left(A, L_{A}\right)$ the $\tilde{\mathbf{k}}$ superalgebra $\mathcal{F}(\mathcal{A})$ is a commutative superalgebra (see Definitions 2.14 and 3.24 ).

In what follows we assume additionally that $\mathbf{A}$ is PID, and we fix an element $h \in \mathbf{A}$ such that $\mathfrak{m}=h \mathbf{A}$.

Let $\mathcal{A}=\left(A, L_{A}\right)$ be an almost commutative $(\mathbf{k}, \mathbf{A})$-superalgebra. The identity $\left[L_{A}, L_{A}\right] \subset h L_{A}$ allows for defining a bilinear map $\{\cdot, \cdot \cdot\}: \mathcal{F}(\mathcal{A}) \times \mathcal{F}(\mathcal{A}) \rightarrow \mathcal{F}(\mathcal{A})$ via:

$$
\left\{l+h L_{A}, l^{\prime}+h L_{A}\right\}:=\frac{1}{h}\left[l, l^{\prime}\right]+h L_{A}
$$

for $l, l^{\prime} \in L_{A}$.

Lemma 3.27. For any almost commutative $(\mathbf{k}, \mathbf{A})$-algebra $\mathcal{A}$ the operation (3.7) is a Poisson bracket on the commutative $\tilde{\mathbf{k}}$-superalgebra $\mathcal{F}(\mathcal{A})$. In the other words, $\mathcal{F}(\mathcal{A})$ is a Poisson superalgebra.

Proof. It suffices to verify the super-Leibniz rule (2.4) and super-Jacobi identity (2.6). For any $a \in A_{\varepsilon}, b \in A_{\delta}, c \in A_{\gamma}$ we abbreviate $\bar{a}=a+\mathfrak{m} L_{A}, \bar{b}=b+\mathfrak{m} L_{A}, \bar{c}=$ $c+\mathfrak{m} L_{A}$. Then

$\{\bar{a}, \bar{b} \bar{c}\}-\{\bar{a}, \bar{b}\} \bar{c}-(-1)^{\varepsilon \delta} \bar{b}\{\bar{a}, \bar{c}\}=\frac{1}{h}\left([a, b c]-[a, b] c-(-1)^{\varepsilon \cdot \delta} b[a, c]\right)+\mathfrak{m} L_{A}=\mathfrak{m} L_{A}$

by (3.5), and

$$
\begin{aligned}
& J(\bar{a}, \bar{b}, \bar{c})=(-1)^{\varepsilon \gamma}\{\bar{a},\{\bar{b}, \bar{c}\}\}+(-1)^{\gamma \delta}\{\bar{c},\{\bar{a}, \bar{b}\}\}+(-1)^{\delta \varepsilon}\{\bar{b},\{\bar{c}, \bar{a}\}\} \\
= & \frac{1}{h^{2}}\left((-1)^{\varepsilon \gamma}[a,[b, c]]+(-1)^{\gamma \delta}[c,[a, b]]+(-1)^{\delta \varepsilon}[b,[c, a]]\right)+\mathfrak{m} L_{A}=\mathfrak{m} L_{A}
\end{aligned}
$$

by (3.6). The lemma is proved.

Let $\mathcal{V}=(V, L)$ be an object in $(\mathbf{k}, \mathbf{A})-\operatorname{Mod}$ and let $\sigma: \mathcal{V} \otimes \mathcal{V} \rightarrow \mathcal{V} \otimes \mathcal{V}$ be an involution in this category. Similar to Definition 2.1 above we define the symmetric and exterior squares of $\mathcal{V}=(V, L)$ by

$$
S_{\sigma}^{2} \mathcal{V}=\operatorname{Ker}(\sigma-i d)=\operatorname{Im}(\sigma+i d), \Lambda^{2} \mathcal{V}=\operatorname{Ker}(\sigma+i d)=\operatorname{Im}(\sigma-i d) .
$$

Then we define the braided symmetric and exterior algebras:

$$
S_{\sigma}(\mathcal{V})=T(\mathcal{V}) /\left\langle\Lambda_{\sigma}^{2} \mathcal{V}\right\rangle, \Lambda_{\sigma}(\mathcal{V})=T(\mathcal{V}) /\left\langle S_{\sigma}^{2} \mathcal{V}\right\rangle
$$

Clearly, both $(\mathbf{k}, \mathbf{A})$-algebras $S_{\sigma}(\mathcal{V})$ and $\Lambda_{\sigma}(\mathcal{V})$ are naturally $\mathbb{Z}_{2}$-graded via:

$$
S_{\sigma}(\mathcal{V})_{\overline{0}}=S_{\sigma}(\mathcal{V}), S_{\sigma}(\mathcal{V})_{\overline{1}}=\{0\}, \Lambda_{\sigma}(\mathcal{V})_{\overline{0}}=\bigoplus_{n \geq 0} \Lambda_{\sigma}(\mathcal{V})_{2 n}, \Lambda_{\sigma}(\mathcal{V})_{\overline{1}}=\bigoplus_{n \geq 0} \Lambda_{\sigma}(\mathcal{V})_{2 n+1}
$$


Next, we will describe all involutions $\sigma$ for which both algebras $S_{\sigma}(\mathcal{V})$ and $\Lambda_{\sigma}(\mathcal{V})$ are almost commutative.

Definition 3.28. Given an object $\mathcal{V}=(V, L)$ in $(\mathbf{k}, \mathbf{A})-\operatorname{Mod}$ and an involution $\sigma: \mathcal{V} \otimes \mathcal{V} \rightarrow \mathcal{V} \otimes \mathcal{V}$. We say that $\sigma$ is almost permutation if $(\sigma-\tau)\left(L \otimes_{\mathbf{A}} L\right) \subset \mathfrak{m} L \otimes_{\mathbf{A}} L$, where $\tau: V \otimes V \rightarrow V \otimes V$ is the permutation of factors.

Lemma 3.29. Let $\mathcal{V}$ be an object of $(\mathbf{k}, \mathbf{A})-\operatorname{Mod}$ and let $\sigma: \mathcal{V} \otimes \mathcal{V} \rightarrow \mathcal{V} \otimes \mathcal{V}$ be an almost permutation. Then:

(a) The $(\mathbf{k}, \mathbf{A})$-algebras $S_{\sigma}(\mathcal{V})$ and $\Lambda_{\sigma}(\mathcal{V})$ are almost commutative.

(b) The $\tilde{\mathbf{k}}$-algebras $\mathcal{F}\left(S_{\sigma}(\mathcal{V})\right)$ and $\mathcal{F}\left(\Lambda_{\sigma}(\mathcal{V})\right)$ are homogeneous super-Poisson.

Proof. Prove (a). First, show that $S_{\sigma}(\mathcal{V})$ is almost commutative. Let us introduce a $\mathbb{Z}_{2}$-grading on the tensor algebra $T(\mathcal{V})$ by setting $T(\mathcal{V})_{\overline{0}}=T(\mathcal{V})$ and $T(\mathcal{V})_{\overline{1}}=\{0\}$. Clearly, the ideal $\left\langle\Lambda_{\sigma}^{2} \mathcal{V}\right\rangle$ is $\mathbb{Z}_{2}$-graded. Moreover, this ideal satisfies the assumptions of Proposition 3.25 with $f\left(l, l^{\prime}\right)=\frac{1}{h}(\tau-\sigma)\left(l \otimes l^{\prime}\right)$ because

$$
l \otimes l^{\prime}-\sigma\left(l \otimes l^{\prime}\right)=\left[l, l^{\prime}\right]+h f\left(l, l^{\prime}\right)
$$

for each $l, l^{\prime} \in L$. Therefore, Proposition 3.25 guarantees that the quotient algebra of $\mathcal{A}=T(\mathcal{V})$ by the ideal $\mathcal{J}=\left\langle\Lambda_{\sigma}^{2} \mathcal{V}\right\rangle$ is almost commutative.

Next, we show that $\Lambda_{\sigma}(\mathcal{V})$ is almost commutative. Let us introduce a $\mathbb{Z}_{2}$-grading on the tensor algebra $T(\mathcal{V})$ by first turning $\mathcal{V}$ into $\mathbb{Z}_{2}$-graded space via $\mathcal{V}_{\overline{0}}=\{0\}$, $\mathcal{V}_{\overline{1}}=\mathcal{V}$, and extending this naturally to each tensor power of $\mathcal{V}$. Clearly, the ideal $\left\langle S_{\sigma}^{2} \mathcal{V}\right\rangle$ is $\mathbb{Z}_{2}$-graded. Moreover, this ideal satisfies the assumptions of Proposition 3.25 with $f\left(l, l^{\prime}\right)=\frac{1}{h}(\sigma-\tau)\left(l \otimes l^{\prime}\right)$ because

$$
l \otimes l^{\prime}+\sigma\left(l \otimes l^{\prime}\right)=\left[l, l^{\prime}\right]+h f\left(l, l^{\prime}\right)
$$

for each $l, l^{\prime} \in L$. Therefore, Proposition 3.25 guarantees that the quotient algebra of $\mathcal{A}=T(\mathcal{V})$ by the ideal $\mathcal{J}=\left\langle S_{\sigma}^{2} \mathcal{V}\right\rangle$ is almost commutative. Part (a) is proved.

Part (b) follows directly from (a) and Lemma 3.27. The lemma is proved.

Let $\mathcal{V}=(V, L)$ be an object of $(\mathbf{k}, \mathbf{A})-\operatorname{Mod}$ and let $\sigma: \mathcal{V} \otimes \mathcal{V} \rightarrow \mathcal{V} \otimes \mathcal{V}$ be an almost permutation. Denote $\bar{V}=\mathcal{F}(\mathcal{V})=L / h L$ and define the map $\mathbf{r}$ by the formula: $\mathbf{r}=\mathcal{F}\left(\frac{1}{h}(\sigma-\tau)\right)$, that is,

$$
\mathbf{r}\left((l+h L) \otimes\left(l^{\prime}+h L^{\prime}\right)\right)=\frac{1}{h}(\tau \circ \sigma-i d)\left(l \otimes l^{\prime}\right)+h L \otimes_{\mathbf{A}} L
$$

for $l, l^{\prime} \in L$. Clearly, $\tau \circ \mathbf{r}=-\mathbf{r} \circ \tau$. Therefore, we can define homogeneous brackets $\{\cdot, \cdot\}_{+}=\{\cdot, \cdot\}_{+, \mathbf{r}}$ on $S(\bar{V})$ and $\{\cdot, \cdot\}_{-}=\{\cdot, \cdot\}_{-, \mathbf{r}}$ on $\Lambda(\bar{V})$ by the formula:

$$
\begin{aligned}
& \left\{\bar{v}, \bar{v}^{\prime}\right\}_{+}=\frac{1}{2}(i d+\tau) \mathbf{r}\left(\bar{v} \otimes \bar{v}^{\prime}\right)=\frac{1}{2} \mathbf{r}\left(\bar{v} \otimes \bar{v}^{\prime}-\bar{v}^{\prime} \otimes \bar{v}\right), \\
& \left\{\bar{v}, \bar{v}^{\prime}\right\}_{-}=\frac{1}{2}(i d-\tau) \mathbf{r}\left(\bar{v} \otimes \bar{v}^{\prime}\right)=\frac{1}{2} \mathbf{r}\left(\bar{v} \otimes \bar{v}^{\prime}+\bar{v}^{\prime} \otimes \bar{v}\right)
\end{aligned}
$$

for $v, v^{\prime} \in \bar{V}$.

Clearly, $\left\{\bar{l}, \bar{l}^{\prime}\right\}_{+}=-\left\{\bar{l}^{\prime}, \bar{l}\right\}_{+}$and $\left\{\bar{l}, \bar{l}^{\prime}\right\}_{-}=\left\{\bar{l}^{\prime}, \bar{l}\right\}_{-}$for any $v, v^{\prime} \in \bar{V}$, and the brackets naturally extend to $S(\bar{V})$ on $\Lambda(\bar{V})$ by the (super)Leibniz rule (see e.g., Definition 2.15).

Denote by $P_{\mathbf{r}}(S(\bar{V}))$ and $P_{\mathbf{r}}(\Lambda(\bar{V}))$ the Poisson closures of the bracketed superalgebras $\left(S(\bar{V}),\{\cdot, \cdot\}_{+}\right)$and $\left(\Lambda(\bar{V}),\{\cdot, \cdot\}_{-}\right)$, respectively (along the lines of Proposition 3.4). 
Recall that the functor $\mathcal{F}:(\mathbf{k}, \mathbf{A})-\operatorname{Mod} \rightarrow \tilde{\mathbf{k}}-\operatorname{Mod}$ defined above is an almost equivalence of tensor categories and for any $(\mathbf{k}, \mathbf{A})$-algebra $\mathcal{A}$ the image $\mathcal{F}(\mathcal{A})$ is a k-algebra.

Theorem 3.30. For every object $\mathcal{V}=(V, L)$ of $(\mathbf{k}, \mathbf{A})-$ Mod and any almost permutation $\sigma: \mathcal{V} \otimes \mathcal{V} \rightarrow \mathcal{V} \otimes \mathcal{V}$ one has a surjective homomorphism of homogeneous super-Poisson $\tilde{\mathbf{k}}$-algebras

$$
P_{\mathbf{r}}(S(\bar{V})) \rightarrow \mathcal{F}\left(S_{\sigma}(\mathcal{V})\right), P_{\mathbf{r}}(\Lambda(\bar{V})) \rightarrow \mathcal{F}\left(\Lambda_{\sigma}(\mathcal{V})\right),
$$

where $\bar{V}=\mathcal{F}(\mathcal{V})=L / h L$.

Proof. We need the following obvious fact.

Lemma 3.31. Let $\mathcal{A}$ be a $(\mathbf{k}, \mathbf{A})$-algebra generated by $\mathcal{V}=(V, L)$ (i.e., there is a surjective homomorphism $T(\mathcal{V}) \rightarrow \mathcal{A})$. Then $\mathcal{F}(\mathcal{A})$ is a $\tilde{\mathbf{k}}$-algebra generated by $\bar{V}=\mathcal{F}(\mathcal{V})=L / h L$.

By Lemma $3.29($ a $), S_{\sigma}(\mathcal{V})$ is an evenly $\mathbb{Z}_{2}$-graded almost commutative $(\mathbf{k}, \mathbf{A})$ algebra generated by $\mathcal{V}=(V, L)$. Hence, by Lemmas 3.29(b) and 3.31, $\mathcal{F}\left(S_{\sigma}(\mathcal{V})\right)$ is an evenly $\mathbb{Z}_{2}$-graded commutative $\tilde{\mathbf{k}}$-superalgebra generated by $\bar{V}$. Therefore, there exists a surjective homomorphism

$$
\varphi_{+}: S(\bar{V}) \rightarrow \mathcal{F}\left(S_{\sigma}(\mathcal{V})\right) .
$$

Note that the restriction of $\varphi_{+}$to $S^{2}(\bar{V})$ is an isomorphism $S^{2}(\bar{V}) \widetilde{\rightarrow} \mathcal{F}\left(S_{\sigma}(\mathcal{V})\right)_{2}$ because

$$
\mathcal{F}\left(S_{\sigma}(\mathcal{V})\right)_{2}=\mathcal{F}\left((\mathcal{V} \otimes \mathcal{V}) / \Lambda_{\sigma}^{2} \mathcal{V}\right)=(\bar{V} \otimes \bar{V}) / \Lambda^{2}(\bar{V})
$$

Similarly, by Lemma $3.29(\mathrm{a}), \Lambda_{\sigma}(\mathcal{V})$ is a $\mathbb{Z}_{2}$-graded almost commutative (k, A)superalgebra generated by $\mathcal{V}=(V, L)$. Hence, by Lemmas 3.29(b) and 3.31, $\mathcal{F}\left(\Lambda_{\sigma}(\mathcal{V})\right)$ is a $\mathbb{Z}_{2}$-graded commutative $\tilde{\mathbf{k}}$-superalgebra generated by $\bar{V}$. Therefore, there exists a surjective homomorphism

$$
\varphi_{-}: \Lambda(\bar{V}) \rightarrow \mathcal{F}\left(\Lambda_{\sigma}(\mathcal{V})\right) .
$$

Note that the restriction of $\varphi_{+}$to $\Lambda^{2}(\bar{V})$ is an isomorphism $\Lambda^{2}(\bar{V}) \widetilde{\rightarrow} \mathcal{F}\left(S_{\sigma}(\mathcal{V})\right)_{2}$ because

$$
\mathcal{F}\left(\Lambda_{\sigma}(\mathcal{V})\right)_{2}=\mathcal{F}\left((\mathcal{V} \otimes \mathcal{V}) / \Lambda_{\sigma}^{2} \mathcal{V}\right)=(\bar{V} \otimes \bar{V}) / \Lambda^{2}(\bar{V})
$$

Clearly, both $\varphi_{+}$and $\varphi_{-}$preserve the even $\mathbb{Z}_{2}$-grading. We need the following obvious fact.

Lemma 3.32. Let $A=\bigoplus_{n>0} A_{n}$ and $B=\bigoplus_{n>0} B_{n}$ be homogeneous bracketed superalgebras (see Definition [3.2). Also, let $\varphi: \bar{A} \rightarrow B$ be an algebra homomorphism preserving the $\mathbb{Z}_{\geq 0}$-grading and such that $\varphi\left(\left\{a, a^{\prime}\right\}\right)=\left\{\varphi(a), \varphi\left(a^{\prime}\right)\right\}$ for all $a, a^{\prime} \in A_{1}$.

Then $\varphi$ is a homomorphism of bracketed superalgebras.

It is easy to see that the Poisson bracket on $\mathcal{F}\left(S_{\sigma}(\mathcal{V})\right)$ given by (3.7) satisfies:

$$
\left\{l+h L, l^{\prime}+h L\right\}:=\frac{1}{h}\left(l \cdot l^{\prime}-l^{\prime} \cdot l\right)+h L \cdot L
$$

for any $l, l^{\prime} \in L$. Note that $l \cdot l^{\prime}=l \otimes l^{\prime}+\Lambda_{\sigma}^{2} L=\sigma\left(l \otimes l^{\prime}\right)+\Lambda_{\sigma}^{2} L$ and $l^{\prime} \cdot l=$ $l^{\prime} \otimes l+\Lambda_{\sigma}^{2} L=\tau\left(l \otimes l^{\prime}\right)+\Lambda_{\sigma}^{2} L$. Therefore,

$$
\left\{l+h L, l^{\prime}+h L\right\}=\mathbf{r}\left((l+h L) \otimes\left(l^{\prime}+h L\right)\right)+\mathcal{F}\left(\Lambda_{\sigma}^{2}(V, L)\right) .
$$


Note that $\mathcal{F}\left(\Lambda_{\sigma}^{2}(V, L)\right)=\Lambda^{2}(\bar{V})$ because $\mathcal{F}(\sigma)=\tau$. On the other hand,

$$
\bar{v} \otimes \bar{v}^{\prime}+\Lambda^{2}(\bar{V})=\frac{1}{2}(i d+\tau)\left(\bar{v} \otimes \bar{v}^{\prime}\right)+\Lambda^{2}(\bar{V})
$$

for any $\bar{v}, \bar{v}^{\prime} \in \bar{V}$. Therefore,

$$
\left\{\bar{v}, \bar{v}^{\prime}\right\}+\Lambda^{2}(\bar{V})=\frac{1}{2}(i d+\tau) \mathbf{r}\left(\bar{v} \otimes \bar{v}^{\prime}\right)+\Lambda^{2}(\bar{V})=\varphi_{+}\left(\left\{\bar{v}, \bar{v}^{\prime}\right\}_{+}\right),
$$

where $\left\{\bar{v}, \bar{v}^{\prime}\right\}_{+}$is given by (3.12). Similarly, in $\mathcal{F}\left(\Lambda_{\sigma}(\mathcal{V})\right)_{2}$ one has:

$$
\left\{\bar{v}, \bar{v}^{\prime}\right\}+S^{2}(\bar{V})=\frac{1}{2}(i d-\tau) \mathbf{r}\left(\bar{v} \otimes \bar{v}^{\prime}\right)+\Lambda^{2}(\bar{V})=\varphi_{-}\left(\left\{\bar{v}, \bar{v}^{\prime}\right\}_{-}\right),
$$

where $\left\{\bar{v}, \bar{v}^{\prime}\right\}_{-}$is given by (3.13).

Hence $\varphi_{+}$and $\varphi_{-}$are homomorphisms of bracketed superalgebras by Lemma 3.32. Hence Proposition 3.4 implies that the homomorphisms of bracketed algebras $\varphi_{+}$and $\varphi_{-}$factor through the respective Poisson closures $P_{\sigma}(S(\bar{V})), P_{\sigma}(\Lambda(\bar{V}))$, and we obtain (3.14). Therefore, Theorem 3.30 is proved.

Now let $\mathbf{k}=\mathbb{C}(q)$ and $\mathbf{A}$ be the algebra of rational the functions in $q$ regular at $q=1$ so that $\tilde{\mathbf{k}}=\mathbb{C}$. As we argued above, $A$ is a local PID, with maximal ideal $\mathfrak{m}=h \mathbf{A}$, where $h=q-1$. Recall that for any objects $U$ and $V$ of $\mathcal{O}_{f}$ we defined in (1.11) the normalized braiding $\sigma_{U, V}: U \otimes V \rightarrow V \otimes U$. In particular, each $\sigma_{V, V}$ is an involution.

Recall that $\mathcal{U}_{q}(\mathfrak{g})=\left(U_{q}(\mathfrak{g}), U_{\mathbf{A}}(\mathfrak{g})\right)$ is a $\operatorname{Hopf}(\mathbf{k}, \mathbf{A})$-algebra as above.

Lemma 3.33. Let $V \in \mathcal{O}_{f}$ and let $L$ be a $\mathbf{A}$-lattice in $V$ such that $L$ is also a $U_{\mathbf{A}}(\mathfrak{g})$-submodule of $V$, i.e., $\mathcal{V}=(V, L)$ is an object of $\mathcal{O}_{f}\left(\mathcal{U}_{q}(\mathfrak{g})\right)$. Then

(a) $\sigma_{V, V}\left(L \otimes_{\mathbf{A}} L\right)=L \otimes_{\mathbf{A}} L$. Therefore, $\sigma_{V, V}$ extends to an involutive $\mathcal{U}_{q}(\mathfrak{g})$ module homomorphism $\sigma_{\mathcal{V}, \mathcal{V}}: \mathcal{V} \otimes \mathcal{V} \rightarrow \mathcal{V} \otimes \mathcal{V}$

(b) $\sigma_{\mathcal{V}, \mathcal{V}}$ is an almost permutation (see Definition 3.28).

(c) In the notation (3.11) we have $\mathbf{r}=r^{-}$, where $r^{-}=\frac{1}{2}(r-\tau(r))$ is the skew-symmetrized classical $r$-matrix defined in (2.5).

Proof. Prove (a). It suffices to show that $\sigma_{V, V}$ preserves $L \otimes_{\mathbf{A}} L$. Indeed, it follows from the definition (1.8) that $D \in U_{\mathbf{A}}(\mathfrak{g}) \widehat{\otimes}_{\mathbf{A}} U_{\mathbf{A}}(\mathfrak{g})$ and $u \otimes v \mapsto D(u \otimes v)$ is a well-defined invertible map $V \otimes_{\mathbf{k}} V \rightarrow V \otimes_{\mathbf{k}} V$. Therefore, $D\left(L \otimes_{\mathbf{A}} L\right)=L \otimes_{\mathbf{A}} L$. We obtain by (1.12):

$$
\sigma_{V, V}\left(L \otimes_{\mathbf{A}} L\right)=\left(\mathcal{R}_{V, V} D^{-1}\right)\left(L \otimes_{\mathbf{A}} L\right)=\mathcal{R}_{V, V}\left(L \otimes_{\mathbf{A}} L\right)=L \otimes_{\mathbf{A}} L
$$

by Lemma 3.19 (c). This proves (a).

Prove (b). Indeed, by (1.11), $\sigma_{V, V}=\left.\tau R D^{-1}\right|_{V \otimes V}$. But, taking into account (1.7) and (1.9), we see that the operator $R D^{-1}-i d$ acts on each $z \in L \otimes_{\mathbf{A}} L$ as an element of $(q-1) U_{\mathbf{A}}(\mathfrak{g})$. This implies that $\left(\sigma_{V, V}-\tau\right)\left(L \otimes_{\mathbf{A}} L\right) \subset(q-1) L \otimes_{\mathbf{A}} L$. Part (b) is proved.

Prove (c). In order to compute $\mathbf{r}=\frac{1}{h} \mathcal{F}(\tau \circ \sigma-i d)$, where $h=q-1, \sigma=\sigma \mathcal{V}, \mathcal{V}$, recall that $\sigma=\tau R D^{-1}$ in the notation of Section 1. Clearly,

$$
\begin{gathered}
\mathcal{F}\left(\frac{1}{h}(R-i d)\right)=r, \mathcal{F}\left(\frac{1}{h}\left(R^{o p}-i d\right)\right)=\tau(r), \\
2 \mathcal{F}\left(\frac{1}{h}(D-i d)\right)=\mathcal{F}\left(\frac{1}{h}\left(D^{2}-i d\right)\right)=\mathcal{F}\left(\frac{1}{h}\left(R^{o p} R-i d\right)\right)=\tau(r)+r .
\end{gathered}
$$


Therefore,

$$
\mathcal{F}\left(\frac{1}{h}(D-i d)\right)=\frac{1}{2}(r+\tau(r))
$$

Finally,

$$
\mathbf{r}=\mathcal{F}\left(\frac{1}{h}(\tau \circ \sigma-i d)\right)=\mathcal{F}\left(\frac{1}{h}\left(R D^{-1}-i d\right)\right)=r-\frac{1}{2}(r+\tau(r))=r^{-} .
$$

The lemma is proved.

Therefore, Theorem 3.30 and Lemma 3.33 guarantee that for $\sigma=\sigma \mathcal{V}, \mathcal{V}$ both algebras $\mathcal{F}\left(S_{\sigma}(\mathcal{V})\right)$ and $\mathcal{F}\left(\Lambda_{\sigma}(\mathcal{V})\right)$ are super-Poisson and we have surjective homomorphisms (3.14). Moreover, Lemma 3.33(c) implies in the brackets (3.12) and (3.13) that the operator $\mathbf{r}$ is now equal to $r^{-}$. This proves that in the notation of Theorem 2.21 and 3.30 we see that $P_{\mathbf{r}}\left(S_{\sigma}(\bar{V})\right)=\overline{S(\bar{V})}, P_{\mathbf{r}}\left(\Lambda_{\sigma}(\bar{V})\right)=\overline{\Lambda(\bar{V})}$ and, therefore, obtain the structural surjective homomorphisms of Poisson superalgebras

$$
\overline{S(\bar{V})} \rightarrow \mathcal{F}\left(S_{\sigma}(\mathcal{V})\right), \overline{\Lambda(\bar{V})} \rightarrow \mathcal{F}\left(\Lambda_{\sigma}(\mathcal{V})\right) .
$$

This proves Theorem 2.21 .

Corollary 3.34 (from the proof of Theorem 2.21). In the notation as above, for each $\mathcal{V}=(V, L)$ in $\mathcal{O}_{f}\left(\mathcal{U}_{q}(\mathfrak{g})\right)$ both the algebras $\mathcal{F}\left(S_{\sigma}(\mathcal{V})\right), \mathcal{F}\left(\Lambda_{\sigma}(\mathcal{V})\right)$ and the structure homomorphisms (3.15) depend only on $\bar{V}$ (but they do not depend on the choice of $V$ in $\mathcal{O}_{f}$ or of the lattice $L$ in $V$ ).

Proof. It follows from Lemma 3.19 that both algebras $\mathcal{F}\left(S_{\sigma}(\mathcal{V})\right)$ and $\mathcal{F}\left(\Lambda_{\sigma}(\mathcal{V})\right)$ depend only on $V$, or, more precisely, on the isomorphism class of $V$ in $\mathcal{O}_{f}$. Therefore, by Corollary 3.22, the above algebras depend (up to an isomorphism) only on the choice of $\bar{V}$ in $\overline{\mathcal{O}}$. By the construction, the structure homomorphisms (3.14) also depend only on $\bar{V}$. Corollary 3.34 is proved.

3.3. Exterior valuations and the proof of Theorem 2.34. Due to Proposition 2.11(a), the assertion $\Lambda_{\sigma}^{n} V_{\ell}=0$ for $n \geq 4$ is equivalent to $\Lambda_{\sigma}\left(V_{\ell}\right)_{n}=0$ for $n \geq 4$ which, in its turn, is equivalent to $\Lambda_{\sigma}\left(V_{\ell}\right)_{4}=0$. By Theorem 2.21] it suffices to prove that ${\overline{\Lambda\left(\bar{V}_{\ell}\right)_{4}}}_{4}=0$, where $\bar{V}_{\ell}$ is the corresponding $\ell+1$-dimensional $g l_{2}(\mathbb{C})$-module, i.e., $\bar{V}_{\ell}$ is the $\ell$-th symmetric power of the standard $g l_{2}(\mathbb{C})$-module $\bar{V}_{1}=\mathbb{C}^{2}$. In its turn, by definition (2.7) of $\overline{\Lambda\left(\bar{V}_{\ell}\right)}$, the latter statement is equivalent to the following one.

Proposition 3.35. For each $\ell \geq 0$ we have

$$
J_{-}\left(\bar{V}_{\ell} \times \bar{V}_{\ell} \times \bar{V}_{\ell}\right) \wedge \bar{V}_{\ell}=\Lambda^{4} \bar{V}_{\ell},
$$

where $J_{-}: \bar{V}_{\ell} \times \bar{V}_{\ell} \times \bar{V}_{\ell} \rightarrow \Lambda^{3}\left(\bar{V}_{\ell}\right)$ is the super-Jacobian map defined in (2.6).

Proof. Recall from Example 2.19 that the super-Poisson bracket on $\Lambda\left(\bar{V}_{\ell}\right)$ is given by:

$$
\{a, b\}_{-}=E(a) \wedge F(b)-F(a) \wedge E(b),
$$

for any $a, b \in \Lambda\left(\bar{V}_{\ell}\right)$.

Recall that the $g l_{2}(\mathbb{C})$-module $\bar{V}_{\ell}$ has a weight basis $\left\{v_{i}, i=0, \ldots, \ell\right\}$ such that

$$
E\left(v_{i}\right)=i v_{i-1}, F\left(v_{i}\right)=(\ell-i) v_{i+1} .
$$


This implies that

$$
\left\{v_{i}, v_{j}\right\}_{-}=i(\ell-j) v_{i-1} \wedge v_{j+1}-j(\ell-i) v_{i+1} \wedge v_{j-1} .
$$

We need the following fact.

Lemma 3.36. For any basis vectors $v_{i}, v_{j}, v_{k} \in V_{\ell}$ one has

$$
\begin{gathered}
J_{-}\left(v_{i}, v_{j}, v_{k}\right)=i(2 j-\ell)(\ell-k) v_{i-1} \wedge v_{j} \wedge v_{k+1}-i(\ell-j)(2 k-\ell) v_{i-1} \wedge v_{j+1} \wedge v_{k} \\
-(2 i-\ell) j(\ell-k) v_{i} \wedge v_{j-1} \wedge v_{k+1}+(2 i-\ell)(\ell-j) k v_{i} \wedge v_{j+1} \wedge v_{k-1} \\
+(\ell-i) j(2 k-\ell) v_{i+1} \wedge v_{j-1} \wedge v_{k}-(\ell-i)(2 j-\ell) k v_{i+1} \wedge v_{j} \wedge v_{k-1} .
\end{gathered}
$$

For each subset $I$ of $[0, \ell]=\{0,1, \ldots, \ell\}$ of the form $I=\left\{i_{1}<i_{2}<\cdots<i_{n}\right\}$ denote by $v_{I} \in \Lambda^{n} \bar{V}_{\ell}$ the monomial $v_{I}:=v_{i_{1}} \wedge v_{i_{2}} \wedge \cdots \wedge v_{i_{n}}$. Clearly for each $n$ the monomials $v_{I},|I|=n$ form a $\mathbb{C}$-linear basis in $\Lambda^{n} \bar{V}_{\ell}$.

Denote by $\mathcal{V}_{n}$ the set of all $n$-element subsets in $[0, \ell]$. We define a total ordering on $\mathcal{V}_{n}$ to be the restriction of the lexicographic one from the set $[0, \ell]^{n}$. For each $n \in[0, \ell]$ define a map $\nu=\nu_{n}: \Lambda^{n} \bar{V}_{\ell} \backslash\{0\} \rightarrow \mathcal{V}_{n}$ uniquely by the formula:

$$
\nu\left(v_{I}\right)=I
$$

for any $0 \leq i_{1}<i_{2}<\cdots<i_{n} \leq \ell$ and $\nu\left(c_{1} u+c_{2} v\right)=\min (\nu(u), \nu(v))$ for any $c_{1}, c_{2} \in \mathbb{C}^{\times}$and $u, v \in \Lambda^{n} \bar{V}_{\ell} \backslash\{0\}$ such that $\nu(u) \neq \nu(v)$ (the minimum is taken in the above ordering of $\mathcal{V}_{n}$ ). We will refer to $\nu$ as a valuation on $\Lambda^{n} \bar{V}_{\ell}$. By definition, $\mathcal{V}_{n}=\nu\left(\Lambda^{n} \bar{V}_{\ell} \backslash\{0\}\right)$.

The following facts are obvious.

Lemma 3.37. If $x \in \Lambda^{n} \bar{V}_{\ell}$ and $y \in \Lambda^{m} \bar{V}_{\ell}$ are such that $\nu(x) \cap \nu(y)=\emptyset$, then $x \wedge y \neq 0$ and $\nu(x \wedge y)=\nu(x) \cup \nu(y)$.

Lemma 3.38. For any $X \subset \Lambda^{n} \bar{V}_{\ell} \backslash\{0\}$ such that $\nu(X)=\mathcal{V}_{n}$ the set $X$ spans $\Lambda^{n} \bar{V}_{\ell}$ as a $\mathbb{C}$-vector space.

Therefore, in order to finish the proof of Proposition 3.35 it suffices to construct a subset $X$ of $V_{\ell} \wedge J_{-}\left(\bar{V}_{\ell} \times \bar{V}_{\ell} \times \bar{V}_{\ell}\right) \backslash\{0\}$ inside $\Lambda^{4} \bar{V}_{\ell} \backslash\{0\}$ such that $\nu(X)=\mathcal{V}_{4}$. We set $X:=X_{1} \cup X_{y} \cup X_{z}$, where $X_{1}, X_{y}$, and $X_{z}$ are defined as follows:

- $X_{1}=\left\{x_{i, j, k, m}, 0 \leq i<j<k \leq \ell: \nu\left(x_{i, j, k, m}\right)=\{i-1, j, k+1, m\}\right\}$, where

$$
x_{i, j, k, m}:=J_{-}\left(v_{i}, v_{j}, v_{k}\right) \wedge v_{m} .
$$

- $X_{y}=\left\{y_{i, j, k}: 0 \leq i<j<k \leq \ell\right\}$, where

$$
y_{i, j, k}=i(2 j-2-\ell) x_{i, j, k, i-1}-j(2 i-\ell) x_{i, j-1, k, i} .
$$

- $X_{z}=\left\{z_{i, j, k}: 0 \leq i<j<k \leq \ell\right\}$, where

$$
z_{i, j, k}=(2 j+2-\ell)(\ell-k) x_{i, j, k, k+1}-(\ell-j)(2 k-\ell) x_{i, j+1, k, k} .
$$

Now we obtain the desirable fact.

Lemma 3.39. In the notation as above we have $\nu(X)=\mathcal{V}_{4}$.

Proof. Clearly, $\nu(X) \supseteq \nu\left(X_{1}\right) \cup \nu\left(X_{y}\right) \cup \nu\left(X_{z}\right)$. Note that for $i^{\prime}<j^{\prime}<k^{\prime}<m^{\prime}$ one has:

$$
\nu\left(x_{i^{\prime}+1, j^{\prime}, m^{\prime}+1, k^{\prime}}\right)=\left\{i^{\prime}, j^{\prime}, k^{\prime}, m^{\prime}\right\}
$$

if $i^{\prime}+1<j^{\prime}, 2 j \neq \ell$, and

$$
\nu\left(x_{i^{\prime}+1, k^{\prime}, m^{\prime}-1, j^{\prime}}\right)=\left\{i^{\prime}, j^{\prime}, k^{\prime}, m^{\prime}\right\}
$$

if $k^{\prime}+1<m^{\prime}, 2 k \neq \ell$. 
Therefore, $\nu\left(X_{1}\right)$ contains the following two subsets:

$$
\left\{\left\{i^{\prime}, j^{\prime}, k^{\prime}, m^{\prime}\right\}: i^{\prime}<j^{\prime}<k^{\prime}<m^{\prime}, 2 j^{\prime} \neq \ell, i^{\prime}+1<j^{\prime}\right\}
$$

and

$$
\left\{\left\{i^{\prime}, j^{\prime}, k^{\prime}, m^{\prime}\right\}: i^{\prime}<j^{\prime}<k^{\prime}<m^{\prime}, 2 k^{\prime} \neq \ell, k^{\prime}+1<m^{\prime}\right\} .
$$

Furthermore, $x_{i, j, k, i-1}=(2 i-\ell) j(\ell-k) v_{i-1} \wedge v_{i} \wedge v_{j-1} \wedge v_{k+1}+$ lower terms and $y_{i, j, k}= \begin{cases}(2 i-\ell) j i(\ell-j)(2 k-\ell) v_{i-1} \wedge v_{i} \wedge v_{j} \wedge v_{k}+\text { lower terms } & \text { if } j<k-1 \\ \delta_{i, j, k} v_{i-1} \wedge v_{i} \wedge v_{k-1} \wedge v_{k}+\text { lower terms } & \text { if } j=k-1\end{cases}$

where $\delta_{i, j, k}=-i(2 i-\ell)(\ell-k+1)((2 k-4-\ell) k+(k-1)(2 k-\ell))$. It is easy to see that $\delta_{i, j, k}=0$ if and only if $2 i=\ell$ because the equation

$$
(2 k-4-\ell) k+(k-1)(2 k-\ell)=(2 k-1)(2 k-\ell-2)-2=0
$$

has no integer solutions $k$ for $\ell>0$. Therefore,

$$
\nu\left(X_{y}\right) \supset \mathcal{V}_{4}^{\prime \prime}=\left\{\left\{i^{\prime}, j^{\prime}, k^{\prime}, m^{\prime}\right\}: i^{\prime}+1=j^{\prime}, 2 j^{\prime}, 2 m^{\prime} \neq \ell\right\} .
$$

Finally, $x_{i, j, k, k+1}=-i(\ell-j)(2 k-\ell) v_{i-1} \wedge v_{j+1} \wedge v_{k} \wedge v_{k+1}+$ lower terms and $z_{i, j, k}=-(\ell-j)(2 k-\ell)(2 i-\ell)(j+1)(\ell-k) v_{i} \wedge v_{j} \wedge v_{k} \wedge v_{k+1}+$ lower terms. Therefore, $X_{z} \supset \mathcal{V}_{4}^{\prime \prime \prime}=\left\{\left\{i^{\prime}, j^{\prime}, k^{\prime}, m^{\prime}\right\}: 2 k^{\prime}, 2 i^{\prime} \neq \ell, m^{\prime}=k^{\prime}+1\right\}$.

This proves the lemma because $\mathcal{V}_{4}^{\prime} \cup \mathcal{V}_{4}^{\prime \prime} \cup \mathcal{V}_{4}^{\prime \prime \prime}=\mathcal{V}_{4}$.

Therefore, Proposition 3.35 is proved.

Therefore, Theorem 2.34 is proved.

3.4. Braided triple products and the proof of Theorem 2.35 . The proof will be rather long and technical. The strategy is as follows. First, we will generalize the relevant submodules of $V_{\ell} \otimes V_{\ell} \otimes V_{\ell}$ to braided triple products inside triple tensor products $V_{\beta_{1}} \otimes V_{\beta_{2}} \otimes V_{\beta_{3}}$ of $U_{q}\left(g l_{2}(\mathbb{C})\right)$-modules and will formulate the appropriate generalization (Theorem 3.40) of Theorem 2.35. The key ingredient in the proof of Theorem 3.40 is Howe duality (cited below in Proposition 4.1) between the highest weight vectors in $V_{\beta_{1}} \otimes V_{\beta_{2}} \otimes V_{\beta_{3}}$ and weight spaces in $U_{q}\left(g l_{3}(\mathbb{C})\right)$-modules. Using the Howe duality, we describe the highest weight vectors of braided triple products as certain subspaces inside these $U_{q}\left(g l_{3}(\mathbb{C})\right)$-modules and then, in Theorems 3.42 and 3.43. establish transversality of these subspaces. Later on, in Proposition 3.51, we establish the real reason for this transversality - the presence of the dual canonical basis in the $U_{q}\left(g l_{3}(\mathbb{C})\right)$-modules. The most technical part of the section is the proof of Theorem 3.42, which is based on a surprising result in combinatorial optimization (Proposition 3.44) that computes the absolute maximum of a certain linear functional on a discrete convex set.

In order to define braided triple products, let us write the decomposition (4.6) from the Appendix for $U_{q}\left(g l_{2}(\mathbb{C})\right)$-modules $V_{\ell_{1}}, V_{\ell_{2}}$ :

$$
V_{\ell_{1}} \otimes V_{\ell_{2}}=\bigoplus_{0 \leq m \leq \min \left(\ell_{1}, \ell_{2}\right)} J_{\ell_{1}, \ell_{2}}^{m}
$$


where $J_{\ell_{1}, \ell_{2}}^{m} \cong V_{\left(\ell_{1}+\ell_{2}-m, m\right)}$. For $\varepsilon \in\{-,+\}$ we define the submodule $V_{\ell_{1}} \bullet_{\varepsilon} V_{\ell_{2}}$ of $V_{\ell_{1}} \otimes V_{\ell_{2}}$ by:

$$
V_{\ell_{1}} \bullet V_{\ell_{2}}:= \begin{cases}\bigoplus_{0 \leq m \leq \min \left(\ell_{1}, \ell_{2}\right), m \in 2 \mathbb{Z}} J_{\ell_{1}, \ell_{2}}^{m} & \text { if } \varepsilon=+, \\ \bigoplus_{0 \leq m \leq \min \left(\ell_{1}, \ell_{2}\right), m \in 2 \mathbb{Z}+1} J_{\ell_{1}, \ell_{2}}^{m} & \text { if } \varepsilon=-.\end{cases}
$$

Clearly, $V_{\ell} \bullet+V_{\ell}=S_{\sigma}^{2} V_{\ell}$ and $V_{\ell} \bullet V_{\ell}=\Lambda_{\sigma}^{2} V_{\ell}$.

Then for any $\beta_{1}, \beta_{2}, \beta_{3} \in \mathbb{Z}_{\geq 0}$ and $\varepsilon_{1}, \varepsilon_{2} \in\{-,+\}$ define the braided triple product $V_{\beta_{1}} \bullet_{\varepsilon_{1}} V_{\beta_{2}} \bullet_{\varepsilon_{2}} V_{\beta_{3}} \subset V_{\beta_{1}} \otimes V_{\beta_{2}} \otimes V_{\beta_{3}}$ by

$$
V_{\beta_{1}} \bullet_{\varepsilon_{1}} V_{\beta_{2}} \bullet_{\varepsilon_{2}} V_{\beta_{3}}:=V_{\beta_{1}} \bullet_{\varepsilon_{1}} V_{\beta_{2}} \otimes V_{\beta_{3}} \cap V_{\beta_{1}} \otimes V_{\beta_{2}} \bullet_{\varepsilon_{2}} V_{\beta_{3}} \text {. }
$$

Clearly, $V_{\beta} \bullet+V_{\beta} \bullet+V_{\beta}=S_{\sigma}^{3} V_{\beta}$ and $V_{\beta} \bullet V_{\beta} \bullet V_{\beta}=\Lambda_{\sigma}^{3} V_{\beta}$.

Theorem 3.40. For any $\beta_{1}, \beta_{2}, \beta_{3} \in \mathbb{Z}_{\geq 0}$ and $\varepsilon \in\{-,+\}$ we have an isomorphism of $U_{q}\left(g l_{2}(\mathbb{C})\right)$-modules:

$$
V_{\beta_{1}} \bullet V_{\beta_{2}} \bullet \varepsilon V_{\beta_{3}} \cong \bigoplus_{\lambda} V_{\lambda}
$$

where the summation is over all $\lambda=\left(\lambda_{1} \geq \lambda_{2}\right)$ such that:

- $\lambda_{1}+\lambda_{2}=\beta_{1}+\beta_{2}+\beta_{3}, \min \left(\lambda_{2}, \beta_{2}\right) \geq\left(\lambda_{2}-\beta_{1}\right)_{+}+\left(\lambda_{2}-\beta_{3}\right)_{+}$,

- $\min \left(\lambda_{2}, \beta_{2}\right)$ is even and $(-1)^{\left(\lambda_{2}-\beta_{1}\right)_{+}}=(-1)^{\left(\lambda_{2}-\beta_{3}\right)_{+}}=\varepsilon$

(with the convention $(a)_{+}:=\max (a, 0)$ for $\left.a \in \mathbb{Z}\right)$.

Proof. The following fact is well-known.

Lemma 3.41. For any $\lambda=\left(\lambda_{1} \geq \lambda_{2} \geq \lambda_{3}\right)$ the restriction of each $U_{q}\left(g l_{3}(\mathbb{C})\right)$ module $V_{\lambda}$ to $U_{q}\left(g l_{2}(\mathbb{C})\right)_{i}, i=1,2$, is multiplicity-free:

$$
\left.V_{\lambda}\right|_{U_{q}\left(g l_{2}(\mathbb{C})\right)_{i}} \cong \bigoplus_{\mu} V_{\mu}^{i}
$$

where each $V_{\mu}^{i}$ is the irreducible $U_{q}\left(g l_{2}(\mathbb{C})_{i}\right.$-submodule the of the highest weigh $\mu=$ $\left(\mu_{1}, \mu_{2}\right)$, and the summation is over all $\mu=\left(\mu_{1}, \mu_{2}\right) \in \mathbb{Z}^{2}$ such that

$$
\lambda_{1} \geq \mu_{1} \geq \lambda_{2} \geq \mu_{2} \geq \lambda_{3} .
$$

Therefore, for each $i \in\{1,2\}$ there exists a unique (up to scalar multiplication) basis $\mathcal{B}_{\lambda}^{i}$ for $V_{\lambda}$ such that the intersection $\mathcal{B}_{\lambda}^{i} \cap V_{\mu}^{i}$ is the (canonical) basis for $V_{\mu}$. Each $b^{i} \in \mathcal{B}_{\lambda}^{i}$ is labeled by a unique Gelfand-Tsetlin pattern $(\mu, \nu)$ where $\mu=\left(\mu_{1}, \mu_{2}\right)$ is such that $b \in V_{\mu}^{i}$ and $\nu \in\left[\mu_{2}, \mu_{1}\right]$ is such that

$$
b_{(\mu, \nu)}^{i}= \begin{cases}F_{1}^{\mu_{1}-\nu}\left(v_{\mu}^{1}\right) & \text { if } i=1, \\ F_{2}^{\nu-\mu_{2}}\left(v_{\mu}^{2}\right) & \text { if } i=2,\end{cases}
$$

where $v_{\mu}^{i}$ is the highest weight vector of $V_{\mu}^{i}$.

We will refer to each $\mathcal{B}_{\lambda}^{i}$ as a Gelfand-Tsetlin basis for $V_{\lambda}$. For $\varepsilon \in\{-,+\}$ and $i \in\{1,2\}$ denote by $\mathcal{B}_{\lambda}^{i}(\beta)^{\varepsilon}$ the set of all $b_{(\mu, \nu)}^{i} \in \mathcal{B}_{\lambda}^{i}(\beta)$ such that $(-1)^{\mu_{2}}=\varepsilon$.

By the construction, $b_{(\mu, \nu)}^{1}$ belongs to $V_{\lambda}(\beta)$, where $\beta_{1}+\beta_{2}+\beta_{3}=\lambda_{1}+\lambda_{2}+\lambda_{3}$, $\mu_{1}+\mu_{2}=\beta_{1}+\beta_{2}, \nu=\beta_{1}$, and $b_{(\mu, \nu)}^{2}$ belongs to $V_{\lambda}(\beta)$, where $\beta_{1}+\beta_{2}+\beta_{3}=$ $\lambda_{1}+\lambda_{2}+\lambda_{3}, \mu_{1}+\mu_{2}=\beta_{2}+\beta_{3}, \nu=\beta_{3}$.

We say that a subset $\mathcal{B}$ of an $m$-dimensional vector space $V$ are in general position if any $m$-element subset of $\mathcal{B}$ is linearly independent. Clearly, if $\mathcal{B} \subset V$ is generic, 
then for any linearly independent subsets $S, S^{\prime} \subset \mathcal{B}$ we have (with the convention that $\langle S\rangle$ is the linear span of $S$ in $V$ )

$$
\operatorname{dim}\left(\langle S\rangle \cap\left\langle S^{\prime}\right\rangle\right)=\max \left(0,|S|+\left|S^{\prime}\right|-m\right) .
$$

Theorem 3.42. For any $\lambda, \beta \in \mathbb{Z}^{3}$ we have: $\mathcal{B}_{\lambda}^{1}(\beta) \cap \mathcal{B}_{\lambda}^{2}(\beta)=\emptyset$, and the union $\mathcal{B}_{\lambda}^{1}(\beta) \cup \mathcal{B}_{\lambda}^{2}(\beta)$ is a generic subset of $V_{\lambda}(\beta)$.

Proof. We will construct a third basis $\mathcal{B}_{\lambda}$ for $V_{\lambda}(\beta)$ with certain convexity properties of the coefficients of the transition matrix between the bases. First we will address a general situation.

For each Laurent polynomial $p \in \mathbb{C}\left[q, q^{-1}\right]$ denote by $\operatorname{deg}(p)$ the maximal degree of $p$. We also denote $\operatorname{deg}(0)=-\infty$.

Theorem 3.43. Let $\mathcal{B}^{i}=\left\{b_{1}^{i}, \ldots, b_{m}^{i}\right\}, i=1,2$, be bases in an m-dimensional vector space $V$ over $\mathbb{C}(q)$ and $\mathcal{B}=\left\{b_{1}, \ldots, b_{m}\right\}$ be another basis in $V$ such that

$$
b_{\ell}^{1}=\sum_{k=1}^{\ell} c_{k \ell}^{1} b_{k}, b_{\ell}^{2}=\sum_{k=1}^{\ell} c_{k \ell}^{2} b_{m+1-k},
$$

where all the coefficients $c_{k \ell}^{i}$ belong to $\mathbb{C}\left[q, q^{-1}\right] \backslash\{0\}$ and satisfy:

- $\operatorname{deg}\left(c_{k+1, \ell}^{i}\right)>\operatorname{deg}\left(c_{k, \ell}^{i}\right)$ for all $1 \leq k<\ell \leq m$.

- $\operatorname{deg}\left(c_{k, \ell}^{i}\right)+\operatorname{deg}\left(c_{k+1, \ell+1}^{i}\right)>\operatorname{deg}\left(c_{k+1, \ell}^{i}\right)+\operatorname{deg}\left(c_{k, \ell+1}^{i}\right)$ for all $1 \leq k<\ell<m$.

Then $\mathcal{B}^{1} \cap \mathcal{B}^{2}=\emptyset$ and the union $\mathcal{B}^{1} \cup \mathcal{B}^{2}$ is a generic subset of $V$.

Proof. First, we will prove a general combinatorial result.

Let $\lambda=\left(0 \leq \lambda_{1} \leq \lambda_{2} \leq \cdots \leq \lambda_{m} \leq n\right) \in \mathbb{Z}^{m}$ be a reversed partition. Denote by $S^{-}=S_{\lambda}^{-}$the set of all $(i, j) \in[1, m] \rightarrow[1, n]$ such that $j \leq \lambda_{i}$ and let $S^{+}=S_{\lambda}^{+}$be the complement of $S^{-}$, i.e., $S^{+}=[1, m] \rightarrow[1, n] \backslash S^{-}$. That is, $S^{+}$is the set of all $(i, j) \in[1, m] \rightarrow[1, n]$ such that $j \geq \lambda_{i}+1$.

We say that an $m \times n$-matrix $A=\left(a_{i j}\right) \in \operatorname{Mat}_{m \times n}(\mathbb{Z})$ is $\lambda$-convex if the matrix coefficients $a_{i, j}$ satisfy

$$
a_{i, j}+a_{i^{\prime}, j^{\prime}}>a_{i^{\prime}, j}+a_{i, j^{\prime}}
$$

for any $i<i^{\prime}$ in $[1, m], j<j^{\prime}$ in $[1, n]$ such that: either $\left\{i, i^{\prime}\right\} \times\left\{j, j^{\prime}\right\} \subset S^{-}$, or $\{i\} \times\left\{j, j^{\prime}\right\} \subset S^{-},\left\{i^{\prime}\right\} \times\left\{j, j^{\prime}\right\} \subset S^{+}$, or $\left\{i, i^{\prime}\right\} \times\left\{j, j^{\prime}\right\} \subset S^{+}$.

Clearly, the set of all $\lambda$-convex matrices is not empty and forms a semigroup (but not a monoid) under the matrix addition.

For any map $\kappa:[1, m] \rightarrow[1, n]$ define the $\kappa$-weight of each $m \times n$-matrix $A$ by

$$
w_{\kappa}(A)=\sum_{i=1}^{m} a_{i, \kappa(i)} .
$$

For each $\kappa:[1, m] \rightarrow[1, n]$ define two multiplicity functions $\mu_{\kappa}^{\varepsilon}:[1, n] \rightarrow \mathbb{Z}_{\geq 0}$, $\varepsilon \in\{-,+\}$ by

$$
\mu_{\kappa}^{\varepsilon}(j)=\left|\left\{i:(i, j) \in S^{\varepsilon}, \kappa(i)=j\right\}\right| .
$$

For any given functions $K^{-}, K^{+}:[1, n] \rightarrow \mathbb{Z}_{\geq 0}$ denote by $\mathcal{F}\left(K^{-}, K^{+}\right)$the set of all maps $\kappa:[1, n] \rightarrow[0, m]$ such that $\mu_{\kappa}^{\varepsilon}=K^{\varepsilon}$ for $\varepsilon \in\{-,+\}$. 
We say that a pair $\left(i, i^{\prime}\right)$ is an inversion of $\kappa:[1, m] \rightarrow[1, n]$ if $1 \leq i<i^{\prime} \leq m$, $\kappa(i)>\kappa\left(i^{\prime}\right)$, and

$$
\mu_{\kappa \circ\left(i, i^{\prime}\right)}^{\varepsilon}=\mu_{\kappa}^{\varepsilon}
$$

for all $\varepsilon \in\{-,+\}$, where $\left(i, i^{\prime}\right):[1, m] \rightarrow[1, m]$ is the transposition interchanging only $i$ and $i^{\prime}$.

Denote by $\operatorname{In}(\kappa) \subset[1, m] \times[1, m]$ the set of all inversions of $\kappa$.

Proposition 3.44. For any $K^{-}, K^{+}:[1, n] \rightarrow \mathbb{Z}_{\geq 0}$ such that $\mathcal{F}\left(K^{-}, K^{+}\right) \neq \emptyset$ we have:

(a) There exists a unique map $\kappa_{\left(K^{-}, K^{+}\right)} \in \mathcal{F}\left(K^{-}, K^{+}\right)$such that $\operatorname{In}\left(\kappa_{\left(K^{-}, K^{+}\right)}\right)$ $=\emptyset$.

(b) For any $\kappa \in \mathcal{F}\left(K^{-}, K^{+}\right) \backslash\left\{\kappa_{\left(K^{-}, K^{+}\right)}\right\}$and any $\lambda$-convex matrix $A$ one has:

$$
w_{\kappa_{\left(K^{-}, K^{+}\right)}}(A)>w_{\kappa}(A) \text {. }
$$

Proof. Prove (a). We need the following result.

Lemma 3.45. Let $\kappa:[1, m] \rightarrow[1, n]$ and $i, i^{\prime} \in[1, m]$. Then:

(a) $\mu_{\kappa \circ\left(i, i^{\prime}\right)}^{\varepsilon}=\mu_{\kappa}^{\varepsilon}$ for all $\varepsilon \in\{-,+\}$ if and only if: either $\left\{i, i^{\prime}\right\} \times\left\{\kappa(i), \kappa\left(i^{\prime}\right)\right\} \subset$ $S^{-}$, or $\{i\} \times\left\{\kappa(i), \kappa\left(i^{\prime}\right)\right\} \subset S^{-},\left\{i^{\prime}\right\} \times\left\{\kappa(i), \kappa\left(i^{\prime}\right)\right\} \subset S^{+}$, or $\left\{i, i^{\prime}\right\} \times\left\{\kappa(i), \kappa\left(i^{\prime}\right)\right\} \subset$ $S^{+}$.

(b) If $\left(i, i^{\prime}\right) \in \operatorname{In}(\kappa)$, then

$$
w_{\kappa \circ\left(i, i^{\prime}\right)}(A)>w_{\kappa}(A)
$$

for any $\lambda$-convex matrix $A$.

Proof. Part (a) is obvious. Prove (b). Recall that $\left(i, i^{\prime}\right) \in \operatorname{In}(\kappa)$ if and only if $\mu_{\kappa \circ\left(i, i^{\prime}\right)}^{\varepsilon}=\mu_{\kappa}^{\varepsilon}$ for all $\varepsilon \in\{-,+\}, i<i^{\prime}, j<j^{\prime}$, where $j=\kappa\left(i^{\prime}\right), i^{\prime}=\kappa(i)$. By the assumption, $i<i^{\prime}, j<j^{\prime}$. Then

$$
w_{\kappa \circ\left(i, i^{\prime}\right)}(A)-w_{\kappa}(A)=a_{i^{\prime}, j^{\prime}}+a_{i, j}-a_{i^{\prime}, j}-a_{i, j^{\prime}}>0
$$

by (a) and the $\lambda$-convexity of $A$. The lemma is proved.

Now let us prove the existence of $\kappa_{0} \in \mathcal{F}\left(K^{-}, K^{+}\right)$with no inversions.

Lemma 3.46. If $\kappa_{0} \in \mathcal{F}\left(K^{-}, K^{+}\right)$satisfies

$$
w_{\kappa_{0}}(A) \geq w_{\kappa}(A)
$$

for all $\kappa \in \mathcal{F}\left(K^{-}, K^{+}\right)$and some $\lambda$-convex matrix $A$, then $\kappa_{0}$ has no inversions. Moreover, if $\kappa \in \mathcal{F}\left(K^{-}, K^{+}\right)$is such that $\operatorname{In}(\kappa) \neq \emptyset$, then there exists $\kappa_{0} \in \mathcal{F}\left(K^{-}, K^{+}\right)$with no inversions such that $w_{\kappa_{0}}(A)>w_{\kappa}(A)$.

Proof. Indeed, let $\kappa_{0} \in \mathcal{F}\left(K^{-}, K^{+}\right)$satisfy (3.26). Then $\kappa_{0}$ has no inversions, because if $\left(i, i^{\prime}\right) \in \operatorname{In}\left(\kappa_{0}\right)$, then Lemma 3.45 would imply that $\kappa_{0} \circ\left(i, i^{\prime}\right) \in \mathcal{F}\left(K^{-}, K^{+}\right)$ and $w_{\kappa_{0} \circ\left(i, i^{\prime}\right)}(A)>w_{\kappa_{0}}(A)$, which contradicts (3.26). Finally, if $\kappa \in \mathcal{F}\left(K^{-}, K^{+}\right)$ and $\operatorname{In}(\kappa) \neq \emptyset$, then Lemma 3.45 implies that there exists $\kappa^{\prime}$ such that $w_{\kappa^{\prime}}(A)>$ $w_{\kappa}(A)$ and then choose $\kappa_{0} \in \mathcal{F}\left(K^{-}, K^{+}\right)$in such a way that $\kappa_{0}$ brings the maximum of the weight $w_{\kappa^{\prime}}(A)$ (such "maximal" $\kappa_{0}$ always exists because the set $\mathcal{F}\left(K^{-}, K^{+}\right)$ is finite). Also, (3.26) implies that $\operatorname{In}\left(\kappa_{0}\right)=\emptyset$ and $w_{\kappa_{0}}(A)>w_{\kappa}(A)$.

Now we prove the uniqueness of such $\kappa_{0}$. We need the following result. 
Lemma 3.47. Assume that $\kappa_{0} \in \mathcal{F}\left(K^{-}, K^{+}\right)$has no inversions. Then $\kappa_{0}(m)$ is uniquely determined by $\left(K^{-}, K^{+}\right)$, or, more precisely,

$$
\kappa_{0}(m)= \begin{cases}k^{-} & \text {if } \exists i_{0} \in[1, m-1]: \sum_{i=\lambda_{i_{0}}+1}^{m} K^{-}(i)=m-i_{0}, \\ k^{+} & \text {otherwise },\end{cases}
$$

where $k^{\varepsilon}=\max \left\{j: K^{\varepsilon}(j) \neq 0\right\}$ for $\varepsilon \in\{+,-\}$.

Proof. Suppose $\kappa_{0} \in \mathcal{F}\left(K^{-}, K^{+}\right)$has no inversion and let us fix any $i^{+} \in[1, m]$ such that $\kappa_{0}\left(i^{+}\right)=k^{+}$and let us fix any $i^{-} \in[1, m]$ such that $\kappa_{0}\left(i^{-}\right)=k^{-}$.

Suppose, by contradiction, that $\kappa_{0}(m) \notin\left\{k^{+}, k^{-}\right\}$, i.e., $i^{-}<m$ and $i^{+}<m$. Then we construct an inversion of $\kappa_{0}$ as follows. If $\left(m, \kappa_{0}(m)\right) \in S^{+}$, then, clearly, $\left(i^{+}, m\right)$ is an inversion of $\kappa_{0}$, and if $\left(m, \kappa_{0}(m)\right) \in S^{-}$, then, clearly, $\left(i^{-}, m\right)$ is an inversion of $\kappa_{0}$. The obtained contradiction proves that $\kappa_{0}(m) \in\left\{k^{+}, k^{-}\right\}$.

Also denote $i_{0, \varepsilon}=\max \left\{i: \kappa_{0}(i)=k^{\varepsilon}\right\}$.

Clearly, $i_{0, \varepsilon}<i_{1, \varepsilon}$ for some $\varepsilon \in\{+,-\}$. Then Lemma 3.45(a) implies that the pair $\left(i_{0, \varepsilon}, i_{1, \varepsilon}\right)$ is an inversion of $\kappa_{0}$.

Therefore $\kappa_{0}(m)$ equals either $k^{+}$or $k^{-}$. Now assume that $\kappa_{0}(m)=k^{-}$. Let $i_{0}<m$ be the largest index such that $\kappa\left(i_{0}\right)=k^{+}$. Since $\kappa_{0}$ has no inversions, we immediately see that $\left(i, \kappa_{0}(i)\right) \in S^{-}$for each $i \in\left[i_{0}, m\right]$. In its turn this implies that $\sum_{i>\lambda_{i_{0}}} K^{-}(i)=m-i_{0}$.

In order to finish the proof we need to show the opposite implication. Now assume that $\sum_{i>\lambda_{i_{0}}} K^{-}(i)=m-i_{0}$ for some $i_{0}<m$. This implies that $\left(i, \kappa_{0}(i)\right) \in S^{-}$ for each $i \in\left[i_{0}, m\right]$. In particular, $\left(m, \kappa_{0}(m)\right) \in S^{-}$, therefore, $\kappa_{0}(m) \neq k^{+}$and, by the above, one must have $\kappa_{0}(m)=k^{-}$. The lemma is proved.

We finish the proof of the uniqueness of $\kappa_{0}$ by induction in $m$. According to Lemma 3.47 if $\kappa_{0} \in \mathcal{F}\left(K^{-}, K^{+}\right)$has no inversions, then $\kappa_{0}(m)$ is uniquely determined by $\left(K^{-}, K^{+}\right)$. It is easy to see that the restriction $\left.\kappa_{0}\right|_{[1, m-1]}$ has no inversions and belongs to $\mathcal{F}\left(L^{-}, L^{+}\right)$, where

$$
L^{\varepsilon}(i)= \begin{cases}K^{\varepsilon}(i) & \text { if } i \neq k^{\varepsilon}, \\ K^{\varepsilon}\left(k^{\varepsilon}\right)-1 & \text { if } i=k^{\varepsilon}, \kappa_{0}(m)=k^{\varepsilon}, \\ K^{\varepsilon}\left(k^{\varepsilon}\right) & \text { if } i=k^{\varepsilon}, \kappa_{0}(m) \neq k^{\varepsilon} .\end{cases}
$$

Since $\left.\kappa_{0}\right|_{[1, m-1]}$ has no inversions and the pair $\left(L^{-}, L^{+}\right)$is uniquely determined by $\left(K^{-}, K^{+}\right)$, it follows that $\left.\kappa_{0}\right|_{[1, m-1]}$ is (by the inductive hypothesis) uniquely determined by $\left(K^{-}, K^{+}\right)$in $\mathcal{F}\left(L^{-}, L^{+}\right)$. This proves (a).

Part (b) follows from (3.26) for $\kappa_{0}=\kappa_{\left(K^{-}, K^{+}\right)}$, which is unique according to the already proved part (a). Therefore, the inequality (3.26) is strict and holds for all $\lambda$-convex matrices $A$.

This proves part (b). Therefore, Proposition 3.44 is proved.

Now we are ready to finish the proof of Theorem 3.43 . For simplicity, we identify $V$ with $\mathbb{C}(q)^{m}$ by means of the basis $\mathcal{B}$. Let $U$ be the $m \times 2 m$ matrix whose columns are $b_{m}^{2}, b_{m-1}^{2}, \ldots, b_{1}^{2} ; b_{1}^{1}, \ldots, b_{m}^{1}$. For each embedding $\tau:[1, m] \hookrightarrow[1,2 m]$ denote $u_{\tau}=u_{1, \tau(1)} \cdots u_{m, \tau(m)}$; and for each $m$-element subset $J \subset[1,2 m]$ denote by $T_{J}$ the set of all bijections $\tau:[1, m] \rightarrow J$ such that $u_{r} \neq 0$. Since each minor is an alternating sum of $u_{\tau}, \tau \in T_{J}$, it suffices to prove that for each $J \subset[1,2 m]$ there 
exists a unique $\tau_{J}$ such that $\operatorname{deg}\left(u_{\tau_{J}}\right)>\operatorname{deg}\left(u_{\tau}\right)$ for any $\tau \in T_{J} \backslash\left\{\tau_{J}\right\}$. Indeed, we will prove it using Proposition 3.44.

First, define an $m \times(m+1)$ integer matrix $A$ by:

$$
a_{k, \ell}=\left\{\begin{array}{ll}
\operatorname{deg}\left(u_{k, \ell}\right) & \text { if } k \geq \ell \\
\operatorname{deg}\left(u_{k, \ell+m-1}\right) & \text { if } k<\ell
\end{array}= \begin{cases}\operatorname{deg}\left(c_{m+1-k, m+1-\ell}^{2}\right) & \text { if } k \geq \ell, \\
\operatorname{deg}\left(c_{k, \ell-1}^{1}\right) & \text { if } k<\ell .\end{cases}\right.
$$

Clearly, $a_{k, \ell}+a_{k+1, \ell+1}>a_{k+1, \ell}+a_{k, \ell+1}$ for all $k, \ell \in[1, m-1]$ such that $\ell-k \notin\{0,1\} ; a_{k, \ell}>a_{k, \ell+1}$ if $k \geq \ell$ and $a_{k, \ell}<a_{k+1, \ell}$ if $\ell>k+1$.

Therefore, if we set $\lambda=(1,2, \ldots, n)$, then $A$ is $\lambda$-convex in the notation (3.22).

Furthermore, given an $m$-element subset $J \subset[1,2 m]$, denote $K^{-}=J \cap[1, m]$ and $K^{+}=\{j-m: j \in J, j>m\}$. We will view these subsets of $[1, m]$ as functions $K^{-}, K^{+}:[1, m] \rightarrow\{0,1\}$. For any embedding $\tau:[1, m] \rightarrow[1,2 m]$ such that $u_{\tau} \neq 0$ we define a map $\varphi(\tau):[1, m] \rightarrow[1, m+1]$ by

$$
\varphi(\tau)(k)= \begin{cases}\tau(k) & \text { if } \tau(k) \leq k \\ \tau(k)+1-m & \text { if } \tau(k) \geq k+m\end{cases}
$$

Clearly, $\varphi(\tau)$ is well-defined. It is easy to see that if $\tau \in T_{J}$, then $\varphi(\tau) \in \mathcal{F}\left(K^{-}, K^{+}\right)$ in the notation of Proposition 3.44 and, moreover, $\varphi: T_{J} \rightarrow \mathcal{F}\left(K^{-}, K^{+}\right)$is a bijection. It is also easy to see that under this bijection we have

$$
\operatorname{deg}\left(u_{\tau}\right)=w_{\varphi(\tau)}(A)
$$

Therefore, by Proposition 3.44, there exists a unique $\tau_{J} \in T_{J}$ such that $\varphi\left(\tau_{J}\right)=$ $\kappa_{\left(K^{-}, K^{+}\right)}$and $\operatorname{deg}\left(u_{\tau_{J}}\right)>\operatorname{deg}\left(u_{\tau}\right)$ for any $\tau \in T_{J} \backslash\left\{\tau_{J}\right\}$. Theorem 3.43 is proved.

Now we will finish the proof of Theorem 3.42. In what follows we fix $\lambda, \beta \in \mathbb{Z}^{3}$ such that $V_{\lambda}(\beta) \neq 0$.

Denote by $\mathcal{B}_{\lambda}$ the dual canonical basis for $V_{\lambda}$ (see e.g., 7, 8, ). Recall from [7, 8] that each element $b \in \mathcal{B}_{\lambda}$ is naturally labeled by a quadruple $\mathbf{m}=\left(m_{1}, m_{2}, m_{12}, m_{21}\right)$ of non-negative integers such that $m_{1} m_{2}=0$, such that $m_{i}+m_{i j} \leq \ell_{i}$ for $\{i, j\}=$ $\{1,2\}$ (here we abbreviated $\ell_{1}=\lambda_{1}-\lambda_{2}, \ell_{2}=\lambda_{2}-\lambda_{3}$ ). For any $\beta \in \mathbb{Z}^{3}$ the intersection $\mathcal{B}_{\lambda}(\beta)=\mathcal{B}_{\lambda} \cap V_{\lambda}(\beta)$ is a basis in $V_{\lambda}(\beta)$ and $b_{\mathbf{m}} \in \mathcal{B}_{\lambda}(\beta)$ if and only if $m_{1} \alpha_{1}+m_{2} \alpha_{2}+\left(m_{12}+m_{21}\right)\left(\alpha_{1}+\alpha_{2}\right)=\lambda-\beta$.

The basis $\mathcal{B}_{\lambda}$ is determined (up to a scalar multiple) by the property that for each $d \geq 0, \beta \in \mathbb{Z}^{3}$, and $i \in\{1,2\}$ a part of $\mathcal{B}_{\lambda}$ spans the subspace $\operatorname{ker} F_{i}^{d+1} \cap V_{\lambda}(\beta)$ and (another) part of $\mathcal{B}_{\lambda}$ spans ker $E_{i}^{d+1} \cap V_{\lambda}(\beta)$.

Lemma $3.48([7,8])$. The action of $E_{i}, F_{i} \in U_{q}\left(g l_{3}\right), i \in\{1,2\}$, on $\mathcal{B}_{\lambda}$ is given by:

$$
E_{i}\left(b_{\mathbf{m}}\right)=\left(m_{i}+m_{j i}\right)_{q} b_{\mathbf{m}-e_{i}}+\left(m_{j i}\right)_{q} b_{\mathbf{m}-e_{i}^{\prime}}+\left(m_{j i}\right)_{q} b_{\mathbf{m}-e_{i}^{\prime \prime}}
$$

for $\{i, j\}=\{1,2\}$, where $e_{1}=(1,0,0,0), e_{1}^{\prime}=(1,0,-1,1), e_{1}^{\prime \prime}=(0,-1,0,1)$, and $e_{2}=(0,1,0,0), e_{2}^{\prime}=(0,1,1,-1), e_{2}^{\prime \prime}=(-1,0,1,0)$. 


$$
F_{i}\left(b_{\mathbf{m}}\right)=\left(m_{j}+m_{i j}^{\prime}\right)_{q} b_{\mathbf{m}+e_{i}^{\prime \prime}}+\left(m_{i j}^{\prime}\right)_{q} b_{\mathbf{m}-f_{i}^{\prime}}+\left(m_{i j}^{\prime}\right)_{q} b_{\mathbf{m}+e_{i}}
$$

for $\{i, j\}=\{1,2\}$, where $f_{1}^{\prime}=(-1,0,0,1), f_{2}^{\prime}=(0,-1,1,0), m_{i j}^{\prime}=\ell_{i}-m_{i}-m_{i j}$.

For $v \in V_{\lambda} \backslash\{0\}$ and $i \in\{1,2\}$ denote

$$
\ell_{i}^{+}(v):=\max \left(k: E_{i}^{k}(v) \neq 0\right\}, \ell_{i}^{-}(v):=\max \left(k: F_{i}^{k}(v) \neq 0\right\} .
$$

It follows from Lemma 3.27 that $\ell_{i}^{+}\left(b_{\mathbf{m}}\right)=m_{i}+m_{j i}$ and $\ell_{i}^{-}\left(b_{\mathbf{m}}\right)=m_{j}+m_{i j}^{\prime}$. Also denote

$$
d_{i}^{-}=d_{i}^{-}(\lambda, \beta)=\min _{v \in V_{\lambda}(\beta) \backslash\{0\}} \ell_{i}^{-}(v), d_{i}^{+}=d_{i}^{+}(\lambda, \beta)=\min _{v \in V_{\lambda}(\beta) \backslash\{0\}} \ell_{i}^{+}(v) .
$$

The properties of $\mathcal{B}_{\lambda}$ established above imply the following simple result.

Lemma 3.49. We have $d_{1}^{+}=d_{1}^{-}+\beta_{2}-\beta_{1}, d_{2}^{+}=d_{2}^{-}+\beta_{3}-\beta_{2}$, and

$$
d_{1}^{-}=\left(\beta_{1}-\lambda_{2}\right)_{+}+\left(\lambda_{2}-\beta_{2}\right)_{+}, d_{2}^{-}=\left(\lambda_{2}-\beta_{3}\right)_{+}+\left(\beta_{2}-\lambda_{2}\right)_{+}
$$

where $(a)_{+}:=\max (a, 0)$ for $a \in \mathbb{Z}$.

Furthermore, we will label the Gelfand-Tsetlin bases $\mathcal{B}_{\lambda}^{i}(\beta)=\mathcal{B}_{\lambda}^{i} \cap V_{\lambda}(\beta), i \in$ $\{1,2\}$, and the dual canonical basis $\mathcal{B}_{\lambda}(\beta):=\mathcal{B}_{\lambda} \cap V_{\lambda}(\beta)$ as follows (with the convention that $\left.m=m_{\lambda, \beta}=\operatorname{dim} V_{\lambda}(\beta)\right)$ :

$$
\mathcal{B}_{\lambda}^{i}(\beta)=\left\{b_{1}^{i}, b_{2}^{i}, \ldots, b_{m}^{i}\right\}, \mathcal{B}_{\lambda}(\beta)=\left\{b_{1}, b_{2}, \ldots, b_{m}\right\},
$$

where $b_{k}^{i}$ (for $k=1,2, \ldots, m, i \in\{1,2\}$ ) is the only element of $\mathcal{B}_{\lambda}^{i}(\beta)$ such that $\ell_{i}^{-}\left(b_{k}^{1}\right)=d_{i}^{-}+k-1$; and $b_{k}$ is the only element of $\mathcal{B}_{\lambda}(\beta)$ such that $\ell_{1}^{-}\left(b_{k}\right)=d_{1}^{-}+k-1$.

Lemma 3.50. The above labeling satisfies for all $\ell \in[1, m]$ :

$$
b_{\ell}^{1} \in \operatorname{Span}\left\{b_{1}, \ldots, b_{\ell}\right\}, b_{\ell}^{2} \in \operatorname{Span}\left\{b_{m-\ell+1}, \ldots, b_{m}\right\} .
$$

Proof. Indeed, by definition, $b_{\ell}^{1} \in \operatorname{Ker} F_{1}^{d_{1}^{-}+\ell} \cap V_{\lambda}(\beta)$. But according to the basic property of $\mathcal{B}_{\lambda}$, the vectors $b_{1}, b_{2}, \ldots, b_{\ell}$ form a basis in $\operatorname{Ker} F_{1}^{d_{1}^{-}+\ell} \cap V_{\lambda}(\beta)$. This proves the first inclusion. To prove the second, it suffices to show that for $d=\ell_{2}^{-}\left(b_{\ell}^{2}\right)$ the intersection $\operatorname{Ker} F_{1}^{d+1} \cap V_{\lambda}(\beta)$ is spanned by $\left\{b_{m-\ell+1}, \ldots, b_{m}\right\}$. That is, we have to show that $\ell_{2}^{-}\left(b_{\ell}^{1}\right) \geq \ell_{2}^{-}\left(b_{k}\right)$ if and only if $k+\ell \geq m+1$.

It is easy to see that for $b_{k} \in V_{\lambda}(\beta)$, we have

$$
b_{k}=b_{\left(m_{1}, m_{2}, m_{12}, m_{21}\right)}
$$

where $m_{1}=\left(\beta_{2}-\lambda_{2}\right)_{+}, m_{2}=\left(\lambda_{2}-\beta_{2}\right)_{+}, m_{12}=\lambda_{1}-\beta_{2}-k-d_{1}^{-}(\lambda, \beta)+1$, $m_{21}=\lambda_{1}-\beta_{1}-m_{1}-m_{12}$. Therefore,

$\ell_{2}^{+}\left(b_{k}\right)=m_{2}+m_{12}=\lambda_{1}-\beta_{2}-k-d_{1}^{-}(\lambda, \beta)+1+\left(\lambda_{2}-\beta_{2}\right)_{+}=d_{2}^{+}(\lambda, \beta)+m-k$ because $m=1+\lambda_{1}-\left(\beta_{1}-\lambda_{2}\right)_{+}-\left(\beta_{3}-\lambda_{2}\right)_{+}-\max \left(\lambda_{2}, \beta_{2}\right)$. Therefore, $\ell_{2}^{-}\left(b_{k}\right)=$ $d_{2}^{-}(\lambda, \beta)+m-k$ and the inequality $\ell_{2}^{-}\left(b_{\ell}^{2}\right) \geq \ell_{2}^{-}\left(b_{k}\right)$ is equivalent to $d_{2}^{-}(\lambda, \beta)+\ell-1 \geq$ $d_{2}^{-}(\lambda, \beta)+m-k$, i.e., $k+\ell \geq m+1$. This proves the second inclusion. The lemma is proved.

The above result implies that $b_{1}^{1}$ is proportional to $b_{1}$ and $b_{1}^{2}$ is proportional to $b_{m}$. In what follows we set $b_{1}^{1}=b_{1}$ and $b_{1}^{2}=b_{m}$ for each $\beta$ such that $d_{1}^{-}(\lambda, \beta)=0$. It is easy to see that this setting agrees with the definition (3.19) of both $\mathcal{B}_{\lambda}^{1}$ and $\mathcal{B}_{\lambda}^{2}$, and, moreover, determines them uniquely.

The following result which completely describes the expansion of Gelfand-Tsetlin bases along the dual canonical basis of $V_{\lambda}(\beta)$ will finish the proof of Theorem 3.42 . 
Proposition 3.51. The bases $\mathcal{B}^{1}:=\mathcal{B}_{\lambda}^{1}(\beta), \mathcal{B}^{2}:=\mathcal{B}_{\lambda}^{2}(\beta)$, and $\mathcal{B}:=\mathcal{B}_{\lambda}(\beta)$ labeled by (3.29) satisfy the hypotheses of Theorem 3.43. More precisely,

$$
b_{\ell}^{1}=\sum_{k=1}^{\ell} c_{k \ell}^{1} b_{k}, b_{\ell}^{2}=\sum_{k=1}^{\ell} c_{k \ell}^{2} b_{m+1-k}
$$

where all the coefficients $c_{k \ell}^{1}, c_{k \ell}^{2}$ belong to $\mathbb{C}\left[q, q^{-1}\right] \backslash\{0\}$ and satisfy the recursion

$$
\operatorname{deg}\left(c_{k, \ell}^{i}\right)-\operatorname{deg}\left(c_{k-1, \ell}^{i}\right)=\left|\beta_{2}-\lambda_{2}\right|+2(\ell-k)+1
$$

for all $1<k \leq \ell \leq m, i \in\{1,2\}$.

Proof. First, the triangularity (3.31) follows from Lemma 3.50. Furthermore, we will establish some symmetries which take Gelfand-Tsetlin bases into each other and preserve the dual canonical basis. The following well-known fact is a particular case of a more general result (see e.g., [8]).

Lemma 3.52. For each $\lambda=\left(\lambda_{1} \geq \lambda_{2} \geq \lambda_{3}\right)$ we have:

(a) There is a unique (up to a scalar multiple) linear isomorphism $\psi_{\lambda}: V_{\lambda} \rightarrow V_{\lambda^{*}}$, where $\lambda^{*}=\left(-\lambda_{3},-\lambda_{2},-\lambda_{1}\right)$ such that

$$
\psi_{\lambda}\left(E_{i}(v)\right)=F_{i}\left(\psi_{\lambda}(v)\right), \psi_{\lambda}\left(F_{i}(v)\right)=E_{i}\left(\psi_{\lambda}(v)\right), \psi_{\lambda}\left(K_{\beta}(v)\right)=K_{-\beta}\left(\psi_{\lambda}(v)\right)
$$

for any $i \in\{1,2\}, v \in V_{\lambda}, \beta \in \mathbb{Z}^{3}$.

(b) there is a unique (up to a scalar multiple) isomorphism $\eta_{\lambda}: V_{\lambda} \rightarrow V_{\lambda}$ such that

$$
\eta_{\lambda}\left(E_{i}(v)\right)=F_{3-i}\left(\eta_{\lambda}(v)\right), \eta_{\lambda}\left(F_{i}(v)\right)=E_{3-i}\left(\eta_{\lambda}(v)\right), \eta_{\lambda}\left(K_{\beta}(v)\right)=K_{\beta^{o p}}\left(\eta_{\lambda}(v)\right)
$$

for any $i \in\{1,2\}, v \in V_{\lambda}, \beta \in \mathbb{Z}^{3}$, where $\left(\beta_{1}, \beta_{2}, \beta_{3}\right)^{o p}=\left(\beta_{3}, \beta_{2}, \beta_{1}\right)$.

(c) $\psi_{\lambda} \circ \eta_{\lambda}=\eta_{\lambda^{*}} \circ \psi_{\lambda^{*}}$.

Clearly, in terms of the labeling (3.29), $\psi_{\lambda}\left(b_{k}^{i}\right)=b_{k}^{i}$ for $i \in\{1,2\}$ for all $k \in[1, m]$ and $\eta_{\lambda}\left(b_{k}^{1}\right)=b_{k}^{2}, \eta_{\lambda}\left(b_{k}^{2}\right)=b_{k}^{1}$ for all $k \in[1, m]$.

The results of [8] imply that the symmetries $\psi_{\lambda}$ and $\eta_{\lambda}$ preserve the dual canonical basis: $\psi_{\lambda}\left(\mathcal{B}_{\lambda}(\beta)\right)=\mathcal{B}_{\lambda^{*}}(-\beta)$ and $\eta_{\lambda}\left(\mathcal{B}_{\lambda}(\beta)\right)=\mathcal{B}_{\lambda}\left(\beta^{o p}\right)$. We can refine this fact as follows.

Lemma 3.53. We have for $k=1,2, \ldots, m$ :

(a) $\psi\left(b_{k}\right)=b_{k}$ for $k=1,2, \ldots, m$.

(b) $\eta_{\lambda}\left(b_{k}\right)=b_{m+1-k}$.

Proof. Prove (a). Using the fact that $\ell_{1}^{-}\left(\psi_{\lambda}(v)\right)=\ell_{1}^{+}(v)$ and $d_{1}^{+}(\lambda, \beta)=d_{1}^{-}\left(\lambda^{*},-\beta\right)$, we see that $\ell_{1}^{-}\left(\psi_{\lambda}\left(b_{k}\right)\right)=d_{1}^{-}\left(\lambda^{*},-\beta\right)+k-1=\ell_{1}^{-}\left(b_{k}\right)$. Therefore, $\psi_{\lambda}\left(b_{k}\right)=b_{k}$.

Part (b) follows follows directly from Lemma 3.50 .

Using the symmetry $\psi_{\lambda}$ we see that it suffices to prove Proposition 3.51 only in the case when $\lambda_{2} \leq \beta_{2}$. On the other hand, using the symmetry $\eta_{\lambda}$ and the property (3.35) for $i=1$ we see that we see that the coefficients $c_{k \ell}^{2}$ also satisfy (3.35).

Therefore, all we have to prove is that in the case when $\beta_{2} \geq \lambda_{2}$ all the coefficients of the first expansion (3.31) satisfy the property (3.35) with $i=1$. In order to achieve this goal, it is convenient to re-label the bases $\mathcal{B}_{\lambda}^{1}(\beta)$ and $\mathcal{B}_{\lambda}(\beta)$ as follows:

$$
\tilde{b}_{\ell}^{1}:=b_{\ell-d_{1}^{-}+1}^{1}, \tilde{b}_{\ell}:=b_{\ell-d_{1}^{-}+1}
$$


for $\ell=d_{1}^{-}, d_{1}^{-}+1, \ldots, D_{1}^{-}$, where $d_{1}^{-}=d_{1}^{-}(\lambda, \beta)=\left(\beta_{1}-\lambda_{2}\right)_{+}$and $D_{1}^{-}=$ $D_{1}^{-}(\lambda, \beta)=\lambda_{1}-\beta_{2}-\left(\beta_{3}-\lambda_{2}\right)_{+} ;$also denote $\tilde{c}_{k, \ell}:=c_{k-d_{1}^{-}+1, \ell-d_{1}^{-}+1}^{1}$.

Then in terms of the this re-labeling it suffices to prove that if $\beta_{2} \geq \lambda_{2}$, then:

$$
\tilde{b}_{\ell}^{1}=\sum_{k=d_{1}^{-}}^{\ell} \tilde{c}_{k \ell} \tilde{b}_{k}
$$

for all $\ell \in\left[d_{1}^{-}, D_{1}^{-}\right]$, where each $\tilde{c}_{k \ell}=\tilde{c}_{k \ell}^{\beta} \in \mathbb{C}\left[q, q^{-1}\right] \backslash\{0\}$ and

$$
\operatorname{deg}\left(\tilde{c}_{k, \ell}^{\beta}\right)-\operatorname{deg}\left(\tilde{c}_{k-1, \ell}^{\beta}\right)=\beta_{2}-\lambda_{2}+2(\ell-k)+1
$$

for all $d_{1}^{-}(\lambda, \beta)<k \leq \ell \leq D_{1}^{-}(\lambda, \beta)$.

Furthermore, we need the following reformulation of Lemma 3.48 .

Lemma 3.54. Let $\beta^{\prime} \in \mathbb{Z}^{3}$ be such that $\lambda_{2} \leq \beta_{2}^{\prime}-1$. Then the action of the operator $E_{1}: V_{\lambda}\left(\beta^{\prime}\right) \rightarrow V_{\lambda}\left(\beta^{\prime}+\alpha_{1}\right)$ relative to the bases $\mathcal{B}_{\lambda}\left(\beta^{\prime}\right)$ and $\mathcal{B}_{\lambda}\left(\beta^{\prime}+\alpha_{1}\right)$ is given by

$$
E_{1}\left(\tilde{b}_{k}\right)=\left(\beta_{2}^{\prime}-\beta_{1}^{\prime}+k\right)_{q} \tilde{b}_{k+1}+\left(\lambda_{2}-\beta_{1}^{\prime}+k\right)_{q} \tilde{b}_{k}
$$

for $k \in\left[d_{1}^{-}\left(\lambda, \beta^{\prime}\right), D_{1}^{-}\left(\lambda, \beta^{\prime}\right)\right]$ (where $\tilde{b}_{k}=0$ in $V_{\lambda}\left(\beta^{\prime}+\alpha_{1}\right)$ if $\left.k=\beta_{1}^{\prime}-\lambda_{2}\right)$.

Proof. First of all (in the notation of Lemma 3.48) we have $m_{2}=0$ and $m_{1}=\beta_{2}^{\prime}-\lambda_{2}$ because $m_{1}-m_{2}=\beta_{2}^{\prime}-\lambda_{2}>0$ and $m_{1} m_{2}=0$.

Therefore, we can rewrite (3.27) as follows:

$E_{1}\left(b_{\left(m_{1}, 0, m_{12}, m_{21}\right)}\right)=\left(m_{1}+m_{21}\right)_{q} b_{\left(m_{1}-1,0, m_{12}, m_{21}\right)}+\left(m_{21}\right)_{q} b_{\left(m_{1}-1,0, m_{12}+1, m_{21}-1\right)}$.

Then, after "translating" the equation $b_{\mathbf{m}}=\tilde{b}_{k}$ in $V_{\lambda}\left(\beta^{\prime}\right)$ (based on (3.30) into

$$
b_{\left(\beta_{2}^{\prime}-\lambda_{2}, 0, \lambda_{1}-\beta_{2}^{\prime}-k, \lambda_{2}-\beta_{1}^{\prime}+k\right)}=\tilde{b}_{k},
$$

we immediately obtain (3.36). The lemma is proved.

We proceed by induction in $\beta$ with respect to the following partial order on $\mathbb{Z}^{3}$ : $\beta \geq \beta^{\prime}$ if $\beta=\beta^{\prime}+n \alpha_{1}$ for some $n \geq 0$. The base of induction consists of all those $\beta$ which satisfy: $V_{\lambda}(\beta) \neq 0$ but $V_{\lambda}\left(\beta-\alpha_{1}\right)=0$. It is easy to see that for such $\beta$ one has $\operatorname{dim} V_{\lambda}(\beta)=1$ and $\beta_{2} \geq \lambda_{2}$ and, therefore, we have nothing to prove. Now we assume that both weight spaces $V_{\lambda}(\beta)$ and $V_{\lambda}\left(\beta-\alpha_{1}\right)$ are non-zero and $\beta_{2} \geq \lambda_{2}$.

Note that if $b_{\ell-1}^{1} \in V_{\lambda}\left(\beta-\alpha_{1}\right)$ and $\ell>\left(\beta_{1}-\lambda_{2}\right)_{+}$, then $b_{\ell}^{1}=E_{1}\left(b_{\ell-1}^{1}\right)$. Therefore, applying (3.36) to (3.34) with $\beta-\alpha_{1}$ and $\ell-1$, we obtain an expansion in $V_{\lambda}(\beta)$ :

$$
\tilde{b}_{\ell}^{1}=\sum_{k<\ell} \tilde{c}_{k, \ell-1}^{\beta^{\prime}} E_{1}\left(\tilde{b}_{k}\right)=\sum_{k<\ell} \tilde{c}_{k, \ell-1}^{\beta^{\prime}}\left(\left(\beta_{2}^{\prime}-\beta_{1}^{\prime}+k\right)_{q} \tilde{b}_{k+1}+\left(\lambda_{2}-\beta_{1}^{\prime}+k\right)_{q} \tilde{b}_{k}\right),
$$

where $\beta^{\prime}=\left(\beta_{1}-1, \beta_{2}+1, \beta_{3}\right)=\beta-\alpha_{1}$. This implies that

$$
\tilde{c}_{k, \ell}^{\beta}=\tilde{c}_{k-1, \ell-1}^{\beta^{\prime}}\left(\beta_{2}-\beta_{1}+k+1\right)_{q}+\tilde{c}_{k, \ell-1}^{\beta^{\prime}}\left(\lambda_{2}-\beta_{1}+k+1\right)_{q}
$$

with the convention that $c_{k, \ell}^{\beta^{\prime}}=0$ for $k=\ell+1$. In particular, this proves that each $c_{k, \ell}^{\beta}$ is a Laurent polynomial in $q$. Then the inductive hypothesis (3.35) taken for $\beta^{\prime}$ and $\ell-1$ implies that for $k<\ell$ the degree of the second summand minus the degree of the first summand equals $2(\ell-k)-1>0$. Therefore,

$$
\operatorname{deg}\left(\tilde{c}_{k, \ell}^{\beta}\right)= \begin{cases}\operatorname{deg}\left(\tilde{c}_{k, \ell-1}^{\beta^{\prime}}\right)+\lambda_{2}-\beta_{1}+k+1 & \text { if } k<\ell, \\ \operatorname{deg}\left(\tilde{c}_{\ell-1, \ell-1}^{\beta^{\prime}}\right)+\beta_{2}-\beta_{1}+\ell+1 & \text { if } k=\ell .\end{cases}
$$


This implies that for $k<\ell$ we have

$$
\begin{gathered}
\operatorname{deg}\left(\tilde{c}_{k, \ell}^{\beta}\right)-\operatorname{deg}\left(\tilde{c}_{k-1, \ell}^{\beta}\right)=\operatorname{deg}\left(\tilde{c}_{k, \ell-1}^{\beta^{\prime}}\right)+\lambda_{2}-\beta_{1}+k+1-\left(\operatorname{deg}\left(\tilde{c}_{k-1, \ell-1}^{\beta^{\prime}}\right)+\lambda_{2}-\beta_{1}+k\right) \\
=1+\operatorname{deg}\left(\tilde{c}_{k, \ell-1}^{\beta^{\prime}}\right)-\operatorname{deg}\left(\tilde{c}_{k-1, \ell-1}^{\beta^{\prime}}\right)=\beta_{2}-\lambda_{2}+2(\ell-k)+1
\end{gathered}
$$

by the same inductive hypothesis. Similarly, for $k=\ell$ we have

$$
\begin{gathered}
\operatorname{deg}\left(\tilde{c}_{\ell, \ell}^{\beta}\right)-\operatorname{deg}\left(\tilde{c}_{\ell-1, \ell}^{\beta}\right)=\operatorname{deg}\left(\tilde{c}_{\ell-1, \ell-1}^{\beta^{\prime}}\right)+\beta_{2}-\beta_{1}+\ell+1-\left(\operatorname{deg}\left(\tilde{c}_{\ell-1, \ell-1}^{\beta^{\prime}}\right)+\lambda_{2}-\beta_{1}+\ell\right) \\
=\lambda_{2}-\beta_{2}+1 .
\end{gathered}
$$

This finishes the induction. The recursion (3.35) is proved. Therefore, the recursion (3.32) with $i=1$ and $\beta_{2} \geq \lambda_{2}$ is proved. Proposition 3.51 is proved.

Therefore, Theorem 3.42 is proved.

Now we will finish the proof of Theorem 3.40

Lemma 3.55. Under the identification $\left(V_{\beta_{1}} \otimes V_{\beta_{2}} \otimes V_{\beta_{3}}\right)^{\lambda}=V_{\lambda}(\beta)$ (in the notation of (2.11)) we have for $\lambda, \beta \in \mathbb{Z}_{\geq 0}^{3}$, and $\varepsilon \in\{-,+\}$ :

$$
\left(\left(V_{\beta_{1}} \bullet V_{\beta_{2}}\right) \otimes V_{\beta_{3}}\right)^{\lambda}=\operatorname{Span}\left\{\mathcal{B}_{\lambda}^{1}(\beta)^{\varepsilon}\right\},\left(V_{\beta_{1}} \otimes\left(V_{\beta_{2}} \bullet V_{\beta_{3}}\right)\right)^{\lambda}=\operatorname{Span}\left\{\mathcal{B}_{\lambda}^{2}(\beta)^{\varepsilon}\right\} .
$$

Proof. In the notation (4.6) each subspace $\left(J_{\beta_{1}, \beta_{2}}^{m} \otimes V_{\beta_{3}}\right)^{\lambda}\left(\operatorname{resp.}\left(V_{\beta_{1}} \otimes J_{\beta_{2}, \beta_{3}}^{m}\right)^{\lambda}\right)$ is spanned by all those $b_{(\mu, \nu)}^{1} \in V_{\lambda}(\beta)$ (resp. $b_{(\mu, \nu)}^{2} \in V_{\lambda}(\beta)$ ) which satisfy $\mu_{2}=m$.

Using the definition (3.16):

$$
V_{\beta_{1}} \bullet+V_{\beta_{2}}=\bigoplus_{m \in 2 \mathbb{Z}} J_{\beta_{1}, \beta_{2}}^{m}, V_{\beta_{1}} \bullet-V_{\beta_{2}}=\bigoplus_{m \in 2 \mathbb{Z}+1} J_{\beta_{1}, \beta_{2}}^{m}
$$

and Lemma 4.3 from Appendix, we finish the proof of Lemma 3.55 .

Furthermore, using Theorem 3.42, Lemma 3.55, and (3.20) with $S=\mathcal{B}_{\lambda}^{1}(\beta)^{\varepsilon}$, $S^{\prime}=\mathcal{B}_{\lambda}^{2}(\beta)^{\varepsilon}$, we obtain

$$
\begin{gathered}
\operatorname{dim}\left(V_{\beta_{1}} \bullet_{\varepsilon} V_{\beta_{2}} \bullet \bullet_{\varepsilon} V_{\beta_{3}}\right)^{\lambda}=\delta_{\lambda_{3}, 0} \cdot \max \left(0,\left|\mathcal{B}_{\lambda}^{1}(\beta)^{\varepsilon}\right|+\left|\mathcal{B}_{\lambda}^{2}(\beta)^{\varepsilon}\right|-\operatorname{dim} V_{\lambda}(\beta)\right) \\
=\delta_{\lambda_{3}, 0} \cdot \max \left(0,\left|\mathcal{B}_{\lambda}^{1}(\beta)^{\varepsilon}\right|-\left|\mathcal{B}_{\lambda}^{2}(\beta)^{-\varepsilon}\right|\right) .
\end{gathered}
$$

Now define a bijection $S_{\lambda}: \mathcal{B}_{\lambda}^{1} \rightarrow \mathcal{B}_{\lambda}^{2}$ by $b_{(\mu, \nu)}^{1} \mapsto b_{\left(\mu^{\prime}, \nu^{\prime}\right)}^{1}$, where

$\mu_{1}^{\prime}=\lambda_{1}-\min \left(\mu_{1}, \nu-\mu_{2}\right), \mu_{2}^{\prime}=\min \left(\lambda_{2}, \mu_{1}+\mu_{2}-\nu\right)-\mu_{2}, \nu^{\prime}=\lambda_{1}+\lambda_{2}+\lambda_{3}-\mu_{1}-\mu_{2}-\mu_{3}$. Clearly, the inverse of $S_{\lambda}$ is given by the same formula and $S_{\lambda}\left(\mathcal{B}_{\lambda}^{1}(\beta)^{\varepsilon}\right)=\mathcal{B}_{\lambda}^{2}(\beta)^{\delta}$, where $\delta=\varepsilon \cdot(-1)^{\min \left(\lambda_{2}, \beta_{2}\right)}$. Therefore, if $\min \left(\lambda_{2}, \beta_{2}\right)$ is odd, then $\left|\mathcal{B}_{\lambda}^{1}(\beta)^{\varepsilon}\right|=$ $\left|\mathcal{B}_{\lambda}^{2}(\beta)^{-\varepsilon}\right|$ and $\left(V_{\beta_{1}} \bullet \varepsilon V_{\beta_{2}} \bullet V_{\beta_{3}}\right)^{\lambda}=0$ for $\varepsilon \in\{-,+\}$. Now assume that $\min \left(\lambda_{2}, \beta_{2}\right)$ is even, in particular, $\left|\mathcal{B}_{\lambda}^{1}(\beta)^{\varepsilon}\right|=\left|\mathcal{B}_{\lambda}^{2}(\beta)^{\varepsilon}\right|$ for $\varepsilon \in\{-,+\}$, and $\lambda_{3}=0$. Therefore,

$$
\operatorname{dim}\left(V_{\beta_{1}} \bullet V_{\beta_{2}} \bullet V_{\beta_{3}}\right)^{\lambda}=\max \left(0,\left|\mathcal{B}_{\lambda}^{1}(\beta)^{\varepsilon}\right|-\left|\mathcal{B}_{\lambda}^{1}(\beta)^{-\varepsilon}\right|\right) .
$$

It is easy to see that $\left|\mathcal{B}_{\lambda}^{1}(\beta)^{\varepsilon}\right|=\left|\left\{m \in \mathbb{Z}: c(\lambda, \beta) \leq m \leq d(\lambda, \beta),(-1)^{m}=\varepsilon\right\}\right|$, where

$$
c(\lambda, \beta)=\max \left(0, \lambda_{2}-\beta_{3}\right), d(\lambda, \beta)=\min \left(\lambda_{2}, \beta_{2}\right)-\max \left(0, \lambda_{2}-\beta_{1}\right) .
$$

Therefore, $\operatorname{dim}\left(V_{\beta_{1}} \bullet \varepsilon V_{\beta_{2}} \bullet V_{\beta_{3}}\right)^{\lambda}=1$ if and only if

$$
(-1)^{\max \left(0, \lambda_{2}-\beta_{3}\right)}=(-1)^{\max \left(0, \lambda_{2}-\beta_{3}\right)}=\varepsilon,
$$

and $\operatorname{dim}\left(V_{\beta_{1}} \bullet V_{\beta_{2}} \bullet V_{\beta_{3}}\right)^{\lambda}=0$ otherwise. Theorem 3.40 is proved. 
To finish the proof of Theorem 2.35 note that in the notation of Theorem 3.40

$$
V_{\ell} \bullet_{\varepsilon} V_{\ell} \bullet_{\varepsilon} V_{\ell} \cong \bigoplus_{\lambda} V_{\lambda}
$$

where the summation is over all $\lambda=\left(\lambda_{1} \geq \lambda_{2} \geq 0\right)$ such that: $\lambda_{1}+\lambda_{2}=3 \ell$ and $(-1)^{\lambda_{2}}=(-1)^{\left(\lambda_{2}-\ell\right)_{+}}=\varepsilon$. Therefore, Theorem 2.35 is proved.

\section{Appendix: Quantum matrices}

The algebra $\mathbb{C}_{q}\left[M_{2 \times 2}\right]$ of quantum $2 \times 2$-matrices is the $Q(q)$-algebra generated by $a, b, c, d$ subject to the relations

$$
c a=q a c, b a=q a b, d c=q c d, d b=q b d, c b=b c, d a-a d=\left(q-q^{-1}\right) b c .
$$

Let $\mathbb{C}_{q}\left[M_{d \times k}\right]$ be the algebra of quantum $m \times n$-matrices, i.e., the $Q(q)$-algebra generated by $x_{i j}, 1 \leq i \leq d, 1 \leq j \leq k$, with the following defining relations: for each $1 \leq i<i^{\prime} \leq d, 1 \leq j<j^{\prime} \leq k$ the subalgebra of $\mathbb{C}_{q}\left[M_{m \times n}\right]$ generated by the four elements $a=x_{i, j}, b=x_{i, j^{\prime}}, c=x_{i^{\prime}, j}, d=x_{i^{\prime}, j^{\prime}}$ is isomorphic to $\mathbb{C}_{q}\left[M_{2 \times 2}\right]$.

The algebra $\mathbb{C}_{q}\left[M_{d \times k}\right]$ is graded by $\mathbb{Z}_{\geq 0}^{d} \times \mathbb{Z}_{\geq 0}^{k}$ via $\operatorname{deg}\left(x_{i j}\right)=e_{i}^{\prime}+e_{j}$, where $e_{1}^{\prime}, \ldots, e_{d}^{\prime}$ (resp. $\left.e_{1}, \ldots, e_{k}\right)$ is the standard basis in $\mathbb{Z}^{d}$ (resp. in $\mathbb{Z}^{k}$ ). That is,

$$
\mathbb{C}_{q}\left[M_{d \times k}\right]=\underset{(\gamma ; \delta) \in \mathbb{Z}_{\geq 0}^{d} \times \mathbb{Z}_{\geq 0}^{k}}{ } \mathbb{C}_{q}\left[M_{d \times k}\right]_{\gamma ; \delta},
$$

where $\mathbb{C}_{q}\left[M_{d \times k}\right]_{\gamma ; \delta}$ is the set of all $x \in \mathbb{C}_{q}\left[M_{d \times k}\right]$ such that $\operatorname{deg}(x)=(\gamma ; \delta)$.

There is a natural action of $U_{q}\left(g l_{d}(\mathbb{C}) \times g l_{k}(\mathbb{C})\right)$ on $\mathbb{C}_{q}\left[M_{d \times k}\right]$ via

$$
E_{i^{\prime}}^{\prime}\left(x_{i, j}\right)=\delta_{i, i^{\prime}} x_{i+1, j}, F_{i^{\prime}}^{\prime}\left(x_{i, j}\right)=\delta_{i-1, i^{\prime}} x_{i-1, j}, K_{\lambda}^{\prime}\left(x_{i, j}\right)=q^{-\lambda_{i}} \cdot x_{i j}
$$

for $i^{\prime}=1,2, \ldots, d-1, \lambda \in \mathbb{Z}_{\geq 0}^{d}$;

$$
E_{j^{\prime}}\left(x_{i j}\right)=\delta_{j^{\prime}, j-1} x_{i, j-1}, F_{j^{\prime}}\left(x_{i j}\right)=\delta_{j, j^{\prime}} x_{i, j+1}, K_{\mu}\left(x_{i, j}\right)=q^{\mu_{j}} x_{i, j}
$$

for $i, i^{\prime}=1,2, \ldots, d-1, j, j^{\prime}=1,2, \ldots, k-1, \lambda \in \mathbb{Z}_{\geq 0}^{d} ;$ and $u(x y)=\mathbf{m}(\Delta(u)(x \otimes y))$ for any $u \in U_{q}\left(g l_{d}(\mathbb{C}) \times g l_{k}(\mathbb{C})\right)$ and $x, y \in \mathbb{C}_{q}\left[M_{d \times k}\right]$, where $\mathbf{m}$ is the multiplication $\mathbb{C}_{q}\left[M_{d \times k}\right] \otimes \mathbb{C}_{q}\left[M_{d \times k}\right] \rightarrow \mathbb{C}_{q}\left[M_{d \times k}\right]$

This definition implies that $K_{\lambda}^{\prime} K_{\mu}(x)=q^{(\mu, \delta)-(\lambda, \gamma)} \cdot x$ for $x \in \mathbb{C}_{q}\left[M_{d \times k}\right]_{\gamma ; \delta}$ and

$$
E_{j}(x y)=E_{j}(x) K_{-\alpha_{j}}(y)+x E_{j}(y), F_{j}(x y)=F_{j}(x) y+K_{\alpha_{j}} x F_{j}(y)
$$

for $j=1,2, \ldots, k-1$.

Simple objects of $\mathcal{O}_{f}$ for $U_{q}\left(g l_{d}(\mathbb{C}) \times g l_{k}(\mathbb{C})\right)$ are labeled by pairs $(\lambda ; \mu)$, where $\lambda$ is a dominant integral $U_{q}\left(g l_{d}(\mathbb{C})\right)$-weight and $\mu$ is a dominant integral $U_{q}\left(g l_{k}(\mathbb{C})\right)$ weight. Each irreducible $U_{q}\left(g l_{d}(\mathbb{C}) \times g l_{k}(\mathbb{C})\right)$-module of the highest weight $(\lambda ; \mu)$ is isomorphic to $V_{\lambda}^{\prime} \otimes V_{\mu}$, where $V_{\lambda}^{\prime}$ is an irreducible $U_{q}\left(g l_{d}(\mathbb{C}) \times g l_{k}(\mathbb{C})\right)$-module trivially extended from that for $U_{q}\left(g l_{d}(\mathbb{C})\right)$, and $V_{\mu}$ is an irreducible $U_{q}\left(g l_{d}(\mathbb{C}) \times g l_{k}(\mathbb{C})\right)$-module trivially extended from that for $U_{q}\left(g l_{k}(\mathbb{C})\right)$.

The decomposition of $\mathbb{C}_{q}\left[M_{d \times k}\right]$ into simple submodules is given by the following

Proposition 4.1 ([21, Theorem 1.1]). If $d \leq k$, then for any $n \geq 0$ one has an isomorphism of $U_{q}\left(g l_{d}(\mathbb{C}) \times g l_{k}(\mathbb{C})\right)$-modules:

$$
\mathbb{C}_{q}\left[M_{d \times k}\right]_{n} \cong \bigoplus_{\lambda} V_{\lambda}^{\prime} \otimes V_{\tilde{\lambda}}
$$


where the summation is over all d-tuples of integers $\lambda=\left(\lambda_{1} \geq \lambda_{2} \geq \cdots \geq \lambda_{d} \geq 0\right)$ such that $\lambda_{1}+\lambda_{2}+\cdots+\lambda_{d}=n$; and $\tilde{\lambda} \in \mathbb{Z}_{\geq 0}^{k}$ stands for the $\lambda$ extended with $k-d$ zeros.

Recall that the braided tensor product $A \otimes_{\mathcal{R}} B$ of algebras $A$ and $B$ in $\mathcal{O}_{g r, f}$ is defined as follows:

As a $U_{q}(\mathfrak{g})$-module, $A \otimes_{\mathcal{R}} B \cong A \otimes B$, and the multiplication is twisted by $\mathcal{R}$ :

$$
(a \otimes b) \cdot\left(a^{\prime} \otimes b^{\prime}\right)=a \mathcal{R}\left(b \otimes a^{\prime}\right) b
$$

for any $a, a^{\prime} \in A, b, b^{\prime} \in B$. Associativity follows from the fact that $\mathcal{R}$ is a braiding.

The following result is an extension of [21, Theorem 1.1].

Proposition 4.2. As a $U_{q}\left(g l_{d}(\mathbb{C})\right)$-module algebra, $\mathbb{C}_{q}\left[M_{d \times k}\right]$ is isomorphic to the braided tensor $k$-th power of the q-polynomial algebra $S_{q}\left[x_{1}, \ldots, x_{d}\right]$ in the braided monoidal category $\mathcal{O}_{g r, f}$ for $U_{q}\left(g l_{d}(\mathbb{C})\right)$. More precisely, the correspondence:

$$
x_{i j} \mapsto 1 \otimes 1 \otimes \cdots \otimes x_{i} \otimes \cdots \otimes 1 \otimes 1,
$$

where $x_{i}$ occurs only in the $j$-th place, $i=1, \ldots, d, j=1, \ldots, k$, defines an isomorphism of algebras in $\mathcal{O}_{g r, f}$ for $U_{q}\left(g l_{d}(\mathbb{C})\right)$ :

$$
\mathbb{C}_{q}\left[M_{d \times k}\right] \widetilde{\rightarrow} \mathbb{C}_{q}\left[x_{1}, \ldots, x_{d}\right] \otimes_{\mathcal{R}} \mathbb{C}_{q}\left[x_{1}, \ldots, x_{d}\right] \otimes_{\mathcal{R}} \cdots \otimes_{\mathcal{R}} \mathbb{C}_{q}\left[x_{1}, \ldots, x_{d}\right] .
$$

Proof. Since both algebras are quadratic, it suffices to show that they have the same quadratic relations. For each $x \in \mathbb{C}_{q}\left[x_{1}, \ldots, x_{d}\right]$ denote $[x]_{j}$ the $k$-tensor of the form $1^{\otimes j-1} \otimes x \otimes 1^{\otimes k-j}$. In particular, the right hand side of (4.2) is $\left[x_{i}\right]_{j}$. Also for $x, y \in \mathbb{C}_{q}\left[x_{1}, \ldots, x_{d}\right]$ and for $1 \leq i<j \leq k$ denote by $[x \otimes y]_{i j}$ the tensor $1^{\otimes i-1} \otimes x \otimes 1^{\otimes j-i-1} \otimes y \otimes 1^{\otimes k-j}$ and extend it by linearity: $\left[\sum_{k} x^{(k)} \otimes y^{(k)}\right]_{i j}=$ $\sum_{k}\left[x^{(k)} \otimes y^{(k)}\right]_{i j}$

Clearly, $\left[x_{j}\right]_{k}\left[x_{i}\right]_{k}=\left[x_{j} x_{i}\right]_{k}=\left[q x_{i} x_{j}\right]_{k}=q\left[x_{i}\right]_{k}\left[x_{j}\right]_{k}$ for any $i<j$ and $\left[x_{i}\right]_{k}\left[x_{j}\right]_{\ell}$ $=\left[\mathcal{R}\left(x_{i} \otimes x_{j}\right)\right]_{k, \ell}$ if $k>\ell$. Taking into account a well-known computation:

$$
\mathcal{R}\left(x_{i} \otimes x_{j}\right)= \begin{cases}x_{j} \otimes x_{i} & \text { if } i<j, \\ q x_{i} \otimes x_{i} & \text { if } i=j, \\ x_{j} \otimes x_{i}+\left(q-q^{-1}\right) x_{i} \otimes x_{j} & \text { if } i>j\end{cases}
$$

we obtain for $k>\ell$

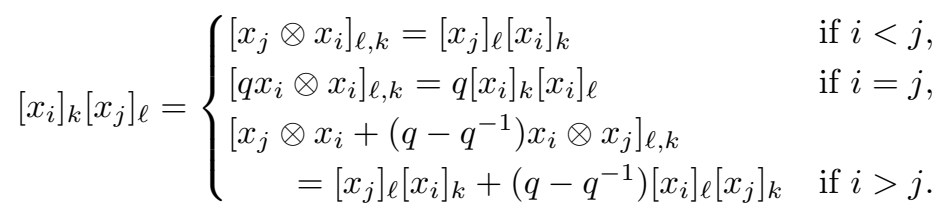

Therefore, the association $x_{i j} \mapsto\left[x_{i}\right]_{j}$ is a surjective homomorphism of algebras. The fact that it is injective follows from the fact that both algebras are flat, i.e., both algebras are isomorphic to $S\left(\mathbb{C}(q)^{d k}\right)$ as graded vector spaces.

According to Proposition 4.1, we have

$$
\left(V_{\beta_{1} \omega_{1}} \otimes \cdots \otimes V_{\beta_{k} \omega_{1}}\right)^{\lambda}=V_{\lambda}(\beta)
$$

for any $\beta=\left(\beta_{1}, \ldots, \beta_{k}\right) \in \mathbb{Z}_{\geq 0}^{k}$, where $V_{\lambda}(\beta)$ is the weight space of the weight $(\beta)$ in the irreducible $U_{q}\left(g l_{k}(\mathbb{C})\right.$ )-module $V_{\lambda}$ in $\mathcal{O}_{f}$, and each $V_{n \omega_{1}} \cong S_{\sigma}^{n} V_{\omega_{1}}$ is a simple $U_{q}\left(g l_{d}(\mathbb{C})\right)$-module. 
By definition, $V_{\ell_{1} \omega_{1}} \otimes V_{\ell_{2} \omega_{1}}$ decomposes into the sum of its isotypic components:

$$
V_{\ell_{1} \omega_{1}} \otimes V_{\ell_{2} \omega_{1}}=\bigoplus_{m=0}^{\min \left(\ell_{1}, \ell_{2}\right)} J_{\ell_{1}, \ell_{2}}^{m},
$$

where $J_{\ell_{1}, \ell_{2}}^{m} \cong V_{\left(\ell_{1}+\ell_{2}-m, m, 0, \ldots, 0\right)}$ as a $U_{q}\left(g l_{k}(\mathbb{C})\right)$-module (with the convention that $J_{\ell_{1}, \ell_{2}}^{m}$ if $\left.m \notin\left[0, \min \left(\ell_{1}, \ell_{2}\right)\right]\right)$.

Lemma 4.3. Under the identification (4.5) we have for each $i \in[1, n-1], \beta=$ $\left(\beta_{1}, \ldots, \beta_{k}\right) \in \mathbb{Z}_{\geq 0}^{k}$, and $m \geq 0$ :

$$
\left(V_{\beta_{1} \omega_{1}} \otimes \cdots \otimes V_{\beta_{i-1} \omega_{1}} \otimes J_{\beta_{i}, \beta_{i+1}}^{m} \otimes \cdots \otimes V_{\beta_{k} \omega_{1}}\right)^{\lambda}=E_{i}^{m}\left(V_{\lambda}\left(\beta-m \alpha_{i}\right) \cap K e r F_{i}\right) .
$$

Proof. We will prove (4.7) by induction in $\beta_{i} \geq 0$. If $\beta_{i}=0$, the statement is obvious because $V_{0} \otimes V_{\beta_{i+1} \omega_{1}}=J_{0, \beta_{i+1}}^{0} \cong V_{\beta_{i+1} \omega_{1}}$ and $V_{\lambda}(\beta) \subset K e r F_{i}$.

It is easy to see that under the identification $\underset{\ell_{1}, \ell_{2} \geq 0}{\bigoplus} V_{\ell_{1} \omega_{1}} \otimes V_{\ell_{2} \omega_{1}}=\mathbb{C}_{q}\left[M_{d \times 2}\right]$, we have $E_{1}\left(J_{\ell_{1}, \ell_{2}}^{m}\right)=J_{\ell_{1}+1, \ell_{2}-1}^{m}$ for all $\ell_{1}, \ell_{2}, m \geq 0$. Therefore, applying $F_{i}$ to (4.7) with $\left(\beta_{1}, \ldots, \beta_{i}-1, \beta_{i+1}+1, \ldots, \beta_{k}\right)$, we obtain (4.7) with $\left(\beta_{1}, \ldots, \beta_{i}, \beta_{i+1}, \ldots, \beta_{k}\right)$.

\section{ACKNOWLEDGMENTS}

We express our gratitude to Joel Kamnitzer and Alexander Polishchuk for very stimulating discussions during the work on the paper.

\section{REFERENCES}

[1] P. Akueson, Geometry of the tangent space on a quantum hyperboloid (French), Cahiers Topologie Géom. Différentielle Catég. 42 (2001), no. 1, 2-50. MR1820764 (2002c:17019)

[2] N. Andruskiewitsch, H.-J. Schneider, Finite Quantum Groups and Cartan Matrices. Adv. Math. 154 (2000), 1-45. MR 1780094 (2001g:16070)

[3] A. Berenstein and D. Kazhdan, Geometric and unipotent crystals, Geom. Funct. Anal. 2000, Special Volume, Part I, 188-236. MR1826254 (2003b:17013)

[4] A. Berenstein and D. Kazhdan, Geometric and unipotent crystals II: from geometric crystals to crystal bases, submitted to Contemporary Mathematics.

[5] A. Berenstein, D. Kazhdan, Lecture notes on geometric crystals and their combinatorial analogues, to appear in Proceedings of the workshop on Combinatorial Aspect of Integrable Systems, RIMS August 2004.

[6] A. Berenstein, D. Kazhdan, Algebro-geometric distributions on reductive groups and geometric crystals, in preparation.

[7] A. Berenstein, A. Zelevinsky, String Bases for Quantum Groups of Type $A_{r}$, Advances in Soviet Math., 16, Part 1 (1993), 51-89. MR1237826 (94g:17019)

[8] A. Berenstein, A. Zelevinsky, Canonical bases for the quantum group of type $A_{r}$, and piecewise-linear combinatorics, Duke Math. J. 82 (1996), no. 3, 473-502. MR.1387682 (97g:17007)

[9] A. Berenstein, A. Zelevinsky, Quantum cluster algebras, Advances in Mathematics, vol. 195, 2 (2005), pp. 405-455. MR2146350 (2006a:20092)

[10] K. Brown and K. Goodearl, Lectures on algebraic quantum groups, Birkhäuser, 2002. MR:1898492 (2003f:16067)

[11] J. Donin, Double quantization on the coadjoint representation of $\mathrm{sl}(n)$, Quantum groups and integrable systems, Part I (Prague, 1997). Czechoslovak J. Phys. 47 (1997), no. 11, 1115-1122. MR1615893 (99h:17019)

[12] M. Durdevic, Z. Oziewicz, Clifford Algebras and Spinors for Arbitrary Braids, Differential geometric methods in theoretical physics (Ixtapa-Zihuatanejo, 1993). Adv. Appl. Clifford Algebras 4 (1994), Suppl. 1, 461-467. MR1337730 (96f:15024) 
[13] V. Drinfel'd, Quasi-Hopf Algebras, Leningrad Math. Journal, 1 (1990), no. 6. MR1047964 (91b:17016)

[14] V. Drinfel'd, Commutation Relations in the Quasi-Classical Case, Selecta Mathematica Sovietica, 11, no.4 (1992) MR1206296

[15] G. Greuel, G. Pfister, and H. Schönemann. Singular 2.0. A Computer Algebra System for Polynomial Computations. Centre for Computer Algebra, University of Kaiserslautern (2001). http://www.singular.uni-kl.de.

[16] A. Joseph, Quantum groups and their primitive ideals, Ergebnisse der Math. (3) 29, SpringerVerlag, Berlin, 1995. MR1315966 (96d:17015)

[17] G. Lusztig, Canonical bases arising from quantized enveloping algebras. J. Amer. Math. Soc. 3 (1990), no. 2, 447-498. MR:1035415 (90m:17023)

[18] S. Majid, Free braided differential calculus, braided binomial theorem, and the braided exponential map, J. Math. Phys. 34 (1993), no. 10, 4843-4856. MR1235979 (94i:58013)

[19] O. Rossi-Doria, A $U_{q}(s l(2))$-representation with no quantum symmetric algebra. Atti Accad. Naz. Lincei Cl. Sci. Fis. Mat. Natur. Rend. Lincei (9) Mat. Appl. 10 (1999), no. 1, 5-9. MR:1768515 (2001f:17029)

[20] S. Woronowicz, Differential Calculus on Matrix Pseudogroups (Quantum Groups), Comm. in Math. Phys. 122 125-170, 1989. MR994499 (90g:58010)

[21] R. Zhang, Howe Duality and the Quantum General Linear Group, Proc. Amer. Math. Soc., Amer. Math. Soc., Providence, RI, 131, no. 9, 2681-1692. MR1974323 (2004b:17036)

[22] S. Zwicknagl, Flat deformations of modules over semisimple Lie algebras, Ph.D. thesis, in preparation.

Department of Mathematics, University of Oregon, Eugene, Oregon 97403

E-mail address: arkadiy@math.uoregon.edu

Department of Mathematics, University of Oregon, Eugene, Oregon 97403

E-mail address: zwicki@noether.uoregon.edu

Current address: Department of Mathematics, University of California, Riverside, California 92521

E-mail address: zwick@math.ucr.edu 